\title{
SYMMETRIC SPECTRA
}

MARK HOVEY, BROOKE SHIPLEY, AND JEFF SMITH

\section{Contents}

Introduction $\quad 150$

Organization $\quad 152$

Acknowledgments $\quad 152$

1. Symmetric spectra 153

1.1. Simplicial sets $\quad 153$

1.2. Symmetric spectra $\quad 154$

1.3. Simplicial structure on $S p^{\Sigma} \quad 156$

1.4. Symmetric $\Omega$-spectra 158

2. The smash product of symmetric spectra 159

2.1. Symmetric sequences 159

2.2. Symmetric spectra 162

2.3. The ordinary category of spectra 165

3. Stable homotopy theory of symmetric spectra 165

$\begin{array}{lr}3.1 . & \text { Stable equivalence } \\ & 166\end{array}$

3.2. Model categories $\quad 172$

$\begin{array}{ll}\text { 3.3. Level structure } & 177\end{array}$

$\begin{array}{lr}\text { 3.4. Stable model category } & 179\end{array}$

4. Comparison with the Bousfield-Friedlander category 185

4.1. Quillen equivalences 185

4.2. The Quillen equivalence 186

$\begin{array}{ll}\text { 4.3. Description of } V & 191\end{array}$

5. Additional properties of symmetric spectra 191

5.1. Level model structure $\quad 192$

$\begin{array}{lr}\text { 5.2. Stable cofibrations } & 196\end{array}$

$\begin{array}{ll}\text { 5.3. Pushout smash product } & 197\end{array}$

5.4. The monoid axiom 201

5.5. Proper model categories 203

5.6. Semistable spectra 204

$\begin{array}{ll}\text { References } & 207\end{array}$

Received by the editors March 31, 1998 and, in revised form, July 7, 1999.

2000 Mathematics Subject Classification. Primary 55P42, 55U10, 55 U35.

The first two authors were partially supported by NSF Postdoctoral Fellowships.

The third author was partially supported by an NSF Grant. 


\section{INTRODUCTION}

Stable homotopy theory studies spectra as the linear approximation to spaces. Here, "stable" refers to the consideration of spaces after inverting the suspension functor. This approach is a general one: one can often create a simpler category by inverting an operation such as suspension. In this paper we study a particularly simple model for inverting such operations which preserves product structures. The combinatorial nature of this model means that it is easily transported, and hence may be useful in extending the methods of stable homotopy theory to other settings.

The idea of a spectrum is a relatively simple one: Freudenthal's suspension theorem implies that the sequence of homotopy classes of maps

$$
[X, Y] \rightarrow[\Sigma X, \Sigma Y] \rightarrow \ldots \rightarrow\left[\Sigma^{n} X, \Sigma^{n} Y\right] \rightarrow \ldots
$$

is eventually constant for finite-dimensional pointed CW-complexes $X$ and $Y$, where $\Sigma X=S^{1} \wedge X$ is the reduced suspension of $X$. This suggests forming a stable category where the suspension functor is an isomorphism. The standard way to do this is to define a spectrum to be a sequence of pointed spaces $X_{n}$ together with structure maps $S^{1} \wedge X_{n} \rightarrow X_{n+1}$. This was first done by Lima [Lim59] and later generalized by Whitehead [Whi62]. The suspension functor is not an isomorphism in the category of spectra, but becomes an isomorphism when we invert the stable homotopy equivalences. The resulting homotopy category of spectra is often called the stable homotopy category and has been extensively studied, beginning with the work of Boardman [Vog70] and Adams [Ada74] and continuing to this day. Notice that this definition of a spectrum can be applied to any situation where one has an operation on a category that one would like to invert; however, this simplest construction does not preserve the smash product structure coming from spaces.

One of the stable homotopy category's basic features is that it is symmetric monoidal. There is a smash product, built from the smash product of pointed spaces and analogous to the tensor product of modules, that is associative, commutative, and unital, up to coherent natural isomorphism. However, the category of spectra defined above is not symmetric monoidal. This has been a sticking point for almost forty years now. Indeed, it was long thought that there could be no symmetric monoidal category of spectra; see [Lew91], where it is shown that a symmetric monoidal category of spectra cannot have all the properties one might like.

Any good symmetric monoidal category of spectra allows one to perform algebraic constructions on spectra that are impossible without such a category. This is extremely important, for example, in the algebraic $K$-theory of spectra. In particular, given a good symmetric monoidal category of spectra, it is possible to construct a homotopy category of monoids (ring spectra) and of modules over a given monoid.

In this paper, we describe a symmetric monoidal category of spectra, called the category of symmetric spectra. The ordinary category of spectra as described above is the category of modules over the sphere spectrum. The sphere spectrum is a monoid in the category of sequences of spaces, but it is not a commutative monoid, because the twist map on $S^{1} \wedge S^{1}$ is not the identity. This explains why the ordinary category of spectra is not symmetric monoidal, just as in algebra where the usual internal tensor product of modules is defined only over a commutative ring. To make the sphere spectrum a commutative monoid, we need to keep track of the twist map, and, more generally, of permutations of coordinates. We therefore define a symmetric spectrum to be a sequence of pointed simplicial sets $X_{n}$ together 
with a pointed action of the permutation group $\Sigma_{n}$ on $X_{n}$ and equivariant structure maps $S^{1} \wedge X_{n} \rightarrow X_{n+1}$. We must also require that the iterated structure maps $S^{p} \wedge X_{n} \rightarrow X_{n+p}$ be $\Sigma_{p} \times \Sigma_{n}$-equivariant. This idea is due to the third author; the first and second authors joined the project later.

At approximately the same time as the third author discovered symmetric spectra, the team of Elmendorf, Kriz, Mandell, and May [EKMM97] also constructed a symmetric monoidal category of spectra, called $S$-modules. Some generalizations of symmetric spectra appear in [MMSS98a]. These many new symmetric monoidal categories of spectra, including $S$-modules and symmetric spectra, are shown to be equivalent in an appropriate sense in [MMSS98b] and [Sch98]. Another symmetric monoidal category of spectra sitting between the approaches of [EKMM97] and of this paper is developed in [DS]. We also point out that symmetric spectra are part of a more general theory of localization of model categories [Hir99]; we have not adopted this approach, but both [Hir99] and [DHK] have influenced us considerably.

Symmetric spectra have already proved useful. In [GH97], symmetric spectra are used to extend the definition of topological cyclic homology from rings to schemes. Similarly, in [Shi], Bökstedt's approach to topological Hochschild homology [Bök85] is extended to symmetric ring spectra, without connectivity conditions. And in [SS], it is shown that any linear model category is Quillen equivalent to a model category of modules over a symmetric ring spectrum.

As mentioned above, since the construction of symmetric spectra is combinatorial in nature it may be applied in many different situations. Given any well-behaved symmetric monoidal model category, such as chain complexes, simplicial sets, or topological spaces, and an endofunctor on it that respects the monoidal structure, one can define symmetric spectra. This more general approach is explored in [Hov98b]. In particular, symmetric spectra may be the logical way to construct a model structure for Voevodsky's stable homotopy of schemes [Voe97].

In this paper, we can only begin the study of symmetric spectra. The most significant loose end is the construction of a model category of commutative symmetric ring spectra; such a model category has been constructed by the third author in work in progress. It would also be useful to have a stable fibrant replacement functor, as the usual construction $Q X$ does not work in general. A good approximation to such a functor is constructed in [Shi].

At present the theory of $S$-modules of [EKMM97] is considerably more developed than the theory of symmetric spectra. Their construction appears to be significantly different from symmetric spectra; however, [Sch98] shows that the two approaches define equivalent stable homotopy categories and equivalent homotopy categories of monoids and modules, as would be expected. Each approach has its own advantages. The category of symmetric spectra is technically much simpler than the $S$-modules of [EKMM97]; this paper is almost entirely self-contained, depending only on some standard results about simplicial sets. As discussed above, symmetric spectra can be built in many different circumstances, whereas $S$-modules appear to be tied to the category of topological spaces. There are also technical differences reflecting the result of [Lew91] that there are limitations on any symmetric monoidal category of spectra. For example, the sphere spectrum $S$ is cofibrant in the category of symmetric spectra, but is not in the category of $S$-modules. On the other hand, every $S$-module is fibrant, a considerable technical advantage. Also, the $S$-modules of [EKMM97] are very well suited to the varying universes that arise 
in equivariant stable homotopy theory, whereas we do not yet know how to realize universes in symmetric spectra. For a first step in this direction see [SS].

\section{ORGANIZATION}

The paper is organized as follows. We choose to work in the category of simplicial sets. In the first section, we define symmetric spectra, give some examples, and establish some basic properties. In Section 2 we describe the closed symmetric monoidal structure on the category of symmetric spectra, and explain why such a structure cannot exist in the ordinary category of spectra. In Section 3 we study the stable homotopy theory of symmetric spectra. This section is where the main subtlety of the theory of symmetric spectra arises: we cannot define stable equivalence by using stable homotopy isomorphisms. Instead, we define a map to be a stable equivalence if it is a cohomology isomorphism for all cohomology theories. The main result of this section is that symmetric spectra, together with stable equivalences and suitably defined classes of stable fibrations and stable cofibrations, form a model category. As expected, the fibrant objects are the $\Omega$-spectra; i.e., symmetric spectra $X$ such that each $X_{n}$ is a Kan complex and the adjoint $X_{n} \rightarrow X_{n+1}^{S^{1}}$ of the structure map is a weak equivalence. In Section 4 , we prove that the stable homotopy theories of symmetric spectra and ordinary spectra are equivalent. More precisely, we construct a Quillen equivalence of model categories between symmetric spectra and the model category of ordinary spectra described in [BF78].

In Section 5 we discuss some of the properties of symmetric spectra. In particular, in Section 5.1, we tie up a loose end from Section 3 by establishing two different model categories of symmetric spectra where the weak equivalences are the level equivalences. We characterize the stable cofibrations of symmetric spectra in Section 5.2. In Section 5.3, we show that the smash product of symmetric spectra interacts with the model structure in the expected way. This section is crucial for the applications of symmetric spectra, and, in particular, is necessary to be sure that the smash product of symmetric spectra does define a symmetric monoidal structure on the stable homotopy category. We establish that symmetric spectra are a proper model category in Section 5.5, and use this to verify the monoid axiom in Section 5.4. The monoid axiom is required to construct model categories of monoids and of modules over a given monoid; see [SS97]. In Section 5.6, we define semistable spectra, which are helpful for understanding the difference between stable equivalences and stable homotopy equivalences.

\section{ACKNOWLEDGMENTS}

The authors would like to thank Dan Christensen, Bill Dwyer, Phil Hirschhorn, Dan Kan, Haynes Miller, John Palmieri, Charles Rezk, and Stefan Schwede for many helpful conversations about symmetric spectra.

Notation. We now establish some notation we will use throughout the paper. Many of the categories in this paper have an enriched Hom as well as a set-valued Hom. To distinguish them: in a category $\mathcal{C}$, the set of maps from $X$ to $Y$ is denoted $\mathcal{C}(X, Y)$; in a simplicial category $\mathcal{C}$, the simplicial set of maps from $X$ to $Y$ is denoted $\operatorname{Map}_{\mathcal{C}}(X, Y)$ or $\operatorname{Map}(X, Y)$; in a category $\mathcal{C}$ with an internal Hom, the object in $\mathcal{C}$ of maps from $X$ to $Y$ is denoted $\operatorname{Hom}_{\mathcal{C}}(X, Y)$ or $\operatorname{Hom}(X, Y)$. In case $\mathcal{C}$ 
is the category of modules over a commutative monoid $S$, we also use $\operatorname{Hom}_{S}(X, Y)$ for the internal Hom.

\section{Symmetric SPECTRA}

In this section we construct the category of symmetric spectra over simplicial sets. We begin this section by recalling the basic facts about simplicial sets in Section 1.1, then we define symmetric spectra in Section 1.2. We describe the simplicial structure on the category of symmetric spectra in Section 1.3. The homotopy category of symmetric $\Omega$-spectra is described in Section 1.4 .

1.1. Simplicial sets. We recall the basics. Consult [May67] or [Cur71] for more details.

The category $\Delta$ has the ordered sets $[n]=\{0,1, \ldots, n\}$ for $n \geq 0$ as its objects and the order preserving functions $[n] \rightarrow[m]$ as its maps. The category of simplicial sets, denoted $\mathcal{S}$, is the category of functors from $\Delta^{\mathrm{op}}$ to the category of sets. The set of $n$-simplices of the simplicial set $X$, denoted $X_{n}$, is the value of the functor $X$ at $[n]$. The standard $n$-simplex $\Delta[n]$ is the contravariant functor $\Delta(-,[n])$. Varying $n$ gives a covariant functor $\Delta[-]: \Delta \rightarrow \mathcal{S}$. By the Yoneda lemma, $\mathcal{S}(\Delta[n], X)=X_{n}$ and the contravariant functor $\mathcal{S}(\Delta[-], X)$ is naturally isomorphic to $X$.

Let $G$ be a discrete group. The category of $G$-simplicial sets is the category $\mathcal{S}^{G}$ of functors from $G$ to $\mathcal{S}$, where $G$ is regarded as a category with one object. A $G$-simplicial set is therefore a simplicial set $X$ with a left simplicial $G$-action, i.e., a homomorphism $G \rightarrow \mathcal{S}(X, X)$.

A basepoint of a simplicial set $X$ is a distinguished 0 -simplex $* \in X_{0}$. The category of pointed simplicial sets and basepoint preserving maps is denoted $\mathcal{S}_{*}$. The simplicial set $\Delta[0]=\Delta(-,[0])$ has a single simplex in each degree and is the terminal object in $\mathcal{S}$. A basepoint of $X$ is the same as a map $\Delta[0] \rightarrow X$. The disjoint union $X_{+}=X \amalg \Delta[0]$ adds a disjoint basepoint to the simplicial set $X$. For example, the 0 -sphere is $S^{0}=\Delta[0]_{+}$. A basepoint of a $G$-simplicial set $X$ is a $G$-invariant 0-simplex of $X$. The category of pointed $G$-simplicial sets is denoted $\mathcal{S}_{*}^{G}$.

The smash product $X \wedge Y$ of the pointed simplicial sets $X$ and $Y$ is the quotient $(X \times Y) /(X \vee Y)$ that collapses the simplicial subset $X \vee Y=X \times * \cup * \times Y$ to a point. For pointed $G$-simplicial sets $X$ and $Y$, let $X \wedge_{G} Y$ be the quotient of $X \wedge Y$ by the diagonal action of $G$. For pointed simplicial sets $X, Y$, and $Z$, there are natural isomorphisms $(X \wedge Y) \wedge Z \cong X \wedge(Y \wedge Z), X \wedge Y \cong Y \wedge X$ and $X \wedge S^{0} \cong X$. In the language of monoidal categories, the smash product is a symmetric monoidal product on the category of pointed simplicial sets. We recall the definition of symmetric monoidal product, but for more details see [ML71, VII] or [Bor94, 6.1].

Definition 1.1.1. A symmetric monoidal product on a category $\mathcal{C}$ is: a bifunctor $\otimes: \mathcal{C} \times \mathcal{C} \rightarrow \mathcal{C}$; a unit $U \in \mathcal{C}$; and coherent natural isomorphisms $(X \otimes Y) \otimes$ $Z \cong X \otimes(Y \otimes Z)$ (the associativity isomorphism), $X \otimes Y \cong Y \otimes X$ (the twist isomorphism), and $U \otimes X \cong X$ (the unit isomorphism). The product is closed if the functor $X \otimes(-)$ has a right adjoint $\operatorname{Hom}(X,-)$ for every $X \in \mathcal{C}$. A (closed) symmetric monoidal category is a category $\mathcal{C}$ with a (closed) symmetric monoidal product. 
Coherence of the natural isomorphisms means that all reasonable diagrams built from the natural isomorphisms also commute [ML71]. When the product is closed, the pairing $\operatorname{Hom}(X, Y): \mathcal{C}$ op $\times \mathcal{C} \rightarrow \mathcal{C}$ is an internal Hom. For example, the smash product on the category $\mathcal{S}_{*}$ of pointed simplicial sets is closed. For $X, Y \in \mathcal{S}_{*}$, the pointed simplicial set of maps from $X$ to $Y$ is $\operatorname{Map}_{\mathcal{S}_{*}}(X, Y)=\mathcal{S}_{*}\left(X \wedge \Delta[-]_{+}, Y\right)$. For pointed $G$-simplicial sets $X$ and $Y$, the simplicial subset of $G$-equivariant pointed maps is $\operatorname{Map}_{G}(X, Y)=\mathcal{S}_{*}^{G}\left(X \wedge \Delta[-]_{+}, Y\right)$.

1.2. Symmetric spectra. Let $S^{1}$ be the simplicial circle $\Delta[1] / \partial \Delta[1]$, obtained by identifying the two vertices of $\Delta[1]$.

Definition 1.2.1. A spectrum is

(1) a sequence $X_{0}, X_{1}, \ldots, X_{n}, \ldots$ of pointed simplicial sets; and

(2) a pointed map $\sigma: S^{1} \wedge X_{n} \rightarrow X_{n+1}$ for each $n \geq 0$.

The maps $\sigma$ are the structure maps of the spectrum. A map of spectra $f: X \rightarrow Y$ is a sequence of pointed maps $f_{n}: X_{n} \rightarrow Y_{n}$ such that the diagram

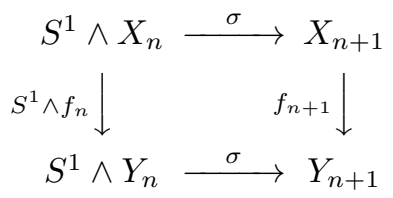

is commutative for each $n \geq 0$. Let $S p^{\mathbb{N}}$ denote the category of spectra.

Replacing the sequence of pointed simplicial sets by a sequence of pointed topological spaces in 1.2.1 gives the original definition of a spectrum (due to Whitehead and Lima). The categories of simplicial spectra and of topological spectra are discussed in the work of Bousfield and Friedlander [BF78].

A symmetric spectrum is a spectrum to which symmetric group actions have been added. Let $\Sigma_{p}$ be the group of permutations of the set $\bar{p}=\{1,2, \ldots, p\}$, with $\overline{0}=\emptyset$. As usual, embed $\Sigma_{p} \times \Sigma_{q}$ as the subgroup of $\Sigma_{p+q}$ with $\Sigma_{p}$ acting on the first $p$ elements of $\overline{p+q}$ and $\Sigma_{q}$ acting on the last $q$ elements of $\overline{p+q}$. Let $S^{p}=\left(S^{1}\right)^{\wedge p}$ be the $p$-fold smash power of the simplicial circle with the left permutation action of $\Sigma_{p}$.

Definition 1.2.2. A symmetric spectrum is

(1) a sequence $X_{0}, X_{1}, \ldots, X_{n}, \ldots$ of pointed simplicial sets;

(2) a pointed map $\sigma: S^{1} \wedge X_{n} \rightarrow X_{n+1}$ for each $n \geq 0$; and

(3) a basepoint preserving left action of $\Sigma_{n}$ on $X_{n}$ such that the composition

$$
\sigma^{p}=\sigma \circ\left(S^{1} \wedge \sigma\right) \circ \cdots \circ\left(S^{p-1} \wedge \sigma\right): S^{p} \wedge X_{n} \rightarrow X_{n+p}
$$

of the maps $S^{i} \wedge S^{1} \wedge X_{n+p-i-1} \stackrel{S^{i} \wedge \sigma}{\longrightarrow} S^{i} \wedge X_{n+p-i}$ is $\Sigma_{p} \times \Sigma_{n}$-equivariant for $p \geq 1$ and $n \geq 0$.

A map of symmetric spectra $f: X \rightarrow Y$ is a sequence of pointed maps $f_{n}: X_{n} \rightarrow Y_{n}$ such that $f_{n}$ is $\Sigma_{n}$-equivariant and the diagram

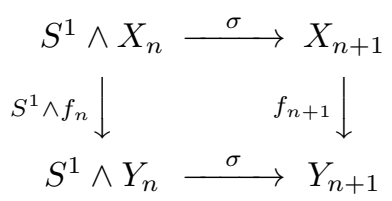

is commutative for each $n \geq 0$. Let $S p^{\Sigma}$ denote the category of symmetric spectra. 
Remark 1.2.3. In part three of Definition 1.2.2, one need only assume that the maps $\sigma: S^{1} \wedge X_{n} \rightarrow X_{n+1}$ and $\sigma^{2}: S^{2} \wedge X_{n} \rightarrow X_{n+2}$ are equivariant; since the symmetric groups $\Sigma_{p}$ are generated by transpositions $(i, i+1)$, if $\sigma$ and $\sigma^{2}$ are equivariant then all the maps $\sigma^{p}$ are equivariant.

Example 1.2.4. The symmetric suspension spectrum $\Sigma^{\infty} K$ of the pointed simplicial set $K$ is the sequence of pointed simplicial sets $S^{n} \wedge K$ with the natural isomorphisms $\sigma: S^{1} \wedge S^{n} \wedge K \rightarrow S^{n+1} \wedge K$ as the structure maps and the diagonal action of $\Sigma_{n}$ on $S^{n} \wedge K$ coming from the left permutation action on $S^{n}$ and the trivial action on $K$. The composition $\sigma^{p}$ is the natural isomorphism which is $\Sigma_{p} \times \Sigma_{n}$-equivariant. The symmetric sphere spectrum $S$ is the symmetric suspension spectrum of the 0-sphere; $S$ is the sequence of spheres $S^{0}, S^{1}, S^{2}, \ldots$ with the natural isomorphisms $S^{1} \wedge S^{n} \rightarrow S^{n+1}$ as the structure maps and the left permutation action of $\Sigma_{n}$ on $S^{n}$.

Example 1.2.5. The Eilenberg-Mac Lane spectrum $H \mathbb{Z}$ is the sequence of simplicial abelian groups $\mathbb{Z} \otimes S^{n}$, where $\left(\mathbb{Z} \otimes S^{n}\right)_{k}$ is the free abelian group on the non-basepoint $k$-simplices of $S^{n}$. We identify the basepoint with 0 . The symmetric group $\Sigma_{n}$ acts by permuting the generators, and one can easily verify that the evident structure maps are equivariant. One could replace $\mathbb{Z}$ by any ring.

Remark 1.2.6. As explained in [GH97, Section 6], many other examples of symmetric spectra arise as the $K$-theory of a category with cofibrations and weak equivalences as defined by Waldhausen [Wal85, p.330].

A symmetric spectrum with values in a simplicial category $\mathcal{C}$ is obtained by replacing the sequence of pointed simplicial sets by a sequence of pointed objects in C. In particular, a topological symmetric spectrum is a symmetric spectrum with values in the simplicial category of topological spaces.

By ignoring group actions, a symmetric spectrum is a spectrum and a map of symmetric spectra is a map of spectra. When no confusion can arise, the adjective "symmetric" may be dropped.

Definition 1.2.7. Let $X$ be a symmetric spectrum. The underlying spectrum $U X$ is the sequence of pointed simplicial sets $(U X)_{n}=X_{n}$ with the same structure maps $\sigma: S^{1} \wedge(U X)_{n} \rightarrow(U X)_{n+1}$ as $X$ but ignoring the symmetric group actions. This gives a faithful functor $U: S p^{\Sigma} \rightarrow S p^{\mathbb{N}}$.

Since the action of $\Sigma_{n}$ on $S^{n}$ is non-trivial for $n \geq 2$, it is usually impossible to obtain a symmetric spectrum from a spectrum by letting $\Sigma_{n}$ act trivially on $X_{n}$. However, many of the usual functors to the category of spectra lift to the category of symmetric spectra. For example, the suspension spectrum of a pointed simplicial set $K$ is the underlying spectrum of the symmetric suspension spectrum of $K$.

Many examples of symmetric spectra and of functors on the category of symmetric spectra are constructed by prolongation of simplicial functors.

Definition 1.2.8. A pointed simplicial functor or $\mathcal{S}_{*}$-functor is a pointed functor $R: \mathcal{S}_{*} \rightarrow \mathcal{S}_{*}$ and a natural transformation $h: R X \wedge K \rightarrow R(X \wedge K)$ of bifunctors such that the composition $R X \wedge S^{0} \rightarrow R\left(X \wedge S^{0}\right) \rightarrow R(X)$ is the unit isomorphism 
and the diagram of natural transformations

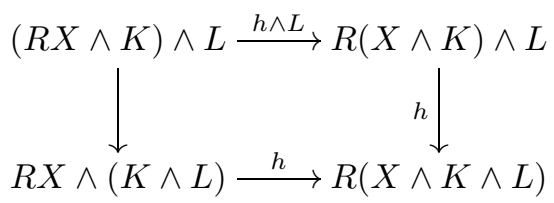

is commutative. A pointed simplicial natural transformation, or $\mathcal{S}_{*}$-natural transformation, from the $\mathcal{S}_{*}$-functor $R$ to the $\mathcal{S}_{*}$-functor $R^{\prime}$ is a natural transformation $\tau: R \rightarrow R^{\prime}$ such that $\tau h=h^{\prime}(\tau \wedge K)$.

Definition 1.2.9. The prolongation of a $\mathcal{S}_{*}$-functor $R: \mathcal{S}_{*} \rightarrow \mathcal{S}_{*}$ is the functor $R: S p^{\Sigma} \rightarrow S p^{\Sigma}$ defined as follows. For $X$ a symmetric spectrum, $R X$ is the sequence of pointed simplicial sets $R X_{n}$ with the composition $\sigma: S^{1} \wedge R\left(X_{n}\right) \rightarrow$ $R\left(S^{1} \wedge X_{n}\right) \stackrel{R \sigma}{\longrightarrow} R\left(X_{n+1}\right)$ as the structure map and the action of $\Sigma_{n}$ on $R\left(X_{n}\right)$ obtained by applying the functor $R$ to the action of $\Sigma_{n}$ on $X_{n}$. Since $R$ is a $\mathcal{S}_{*^{-}}$ functor, each map $\sigma^{p}$ is equivariant and so $R X$ is a symmetric spectrum. For $f$ a map of symmetric spectra, $R f$ is the sequence of pointed maps $R f_{n}$. Since $R$ is an $\mathcal{S}_{*}$-functor, $R f$ is a map of spectra. Similarly, we can prolong an $\mathcal{S}_{*}$-natural transformation to a natural transformation of functors on $S p^{\Sigma}$.

Proposition 1.2.10. The category of symmetric spectra is bicomplete (every small diagram has a limit and a colimit).

Proof. For any small category $I$, the limit and colimit functors $\mathcal{S}_{*}^{I} \rightarrow \mathcal{S}_{*}$ are pointed simplicial functors; for $K \in \mathcal{S}_{*}$ and $D \in$ Set $^{I}$ there is a natural isomorphism

$$
K \wedge \operatorname{colim} D \cong \operatorname{colim}(K \wedge D)
$$

and a natural map

$$
K \wedge \lim D \rightarrow \lim (K \wedge D) .
$$

A slight generalization of prolongation gives the limit and the colimit of a diagram of symmetric spectra.

In particular, the underlying sequence of the $\operatorname{limit}$ is $(\lim D)_{n}=\lim D_{n}$ and the underlying sequence of the colimit is $(\operatorname{colim} D)_{n}=\operatorname{colim} D_{n}$.

1.3. Simplicial structure on $S p^{\Sigma}$. For a pointed simplicial set $K$ and a symmetric spectrum $X$, prolongation of the $\mathcal{S}_{*}$-functor $(-) \wedge K: \mathcal{S}_{*} \rightarrow \mathcal{S}_{*}$ defines the smash product $X \wedge K$ and prolongation of the $\mathcal{S}_{*}$-functor $(-)^{K}: \mathcal{S}_{*} \rightarrow \mathcal{S}_{*}$ defines the power spectrum $X^{K}$. For symmetric spectra $X$ and $Y$, the pointed simplicial set of maps from $X$ to $Y$ is $\operatorname{Map}_{S p^{\Sigma}}(X, Y)=S p^{\Sigma}\left(X \wedge \Delta[-]_{+}, Y\right)$.

In the language of enriched category theory, the following proposition says that the smash product $X \wedge K$ is a closed action of $\mathcal{S}_{*}$ on $S p^{\Sigma}$. We leave the straightforward proof to the reader.

Proposition 1.3.1. Let $X$ be a symmetric spectrum. Let $K$ and $L$ be pointed simplicial sets.

(1) There are coherent natural isomorphisms $X \wedge(K \wedge L) \cong(X \wedge K) \wedge L$ and $X \wedge S^{0} \cong X$

(2) $(-) \wedge K: S p^{\Sigma} \rightarrow S p^{\Sigma}$ is the left adjoint of the functor $(-)^{K}: S p^{\Sigma} \rightarrow S p^{\Sigma}$.

(3) $X \wedge(-): \mathcal{S}_{*} \rightarrow S p^{\Sigma}$ is the left adjoint of the functor $\operatorname{Map}_{S p^{\Sigma}}(X,-): S p^{\Sigma} \rightarrow$ $\mathcal{S}_{*}$. 
The evaluation map $X \wedge \operatorname{Map}_{S p^{\Sigma}}(X, Y) \rightarrow Y$ is the adjoint of the identity map on $\operatorname{Map}_{S p^{\Sigma}}(X, Y)$. The composition pairing

$$
\operatorname{Map}_{S p^{\Sigma}}(X, Y) \wedge \operatorname{Map}_{S p^{\Sigma}}(Y, Z) \rightarrow \operatorname{Map}_{S p^{\Sigma}}(X, Z)
$$

is the adjoint of the composition

$$
X \wedge \operatorname{Map}_{S p^{\Sigma}}(X, Y) \wedge \operatorname{Map}_{S p^{\Sigma}}(Y, Z) \rightarrow Y \wedge \operatorname{Map}_{S p^{\Sigma}}(Y, Z) \rightarrow Z
$$

of two evaluation maps. In the language of enriched category theory, a category with a closed action of $\mathcal{S}_{*}$ is the same as a tensored and cotensored $\mathcal{S}_{*}$-category. The following proposition, whose proof we also leave to the reader, expresses this fact.

Proposition 1.3.2. Let $X, Y$, and $Z$ be symmetric spectra and let $K$ be a pointed simplicial set.

(1) The composition pairing $\operatorname{Map}_{S p^{\Sigma}}(X, Y) \wedge \operatorname{Map}_{S p^{\Sigma}}(Y, Z) \rightarrow \operatorname{Map}_{S p^{\Sigma}}(X, Z)$ is associative.

(2) The adjoint $S^{0} \rightarrow \operatorname{Map}_{S p^{\Sigma}}(X, X)$ of the isomorphism $X \wedge S^{0} \rightarrow X$ is a left and a right unit of the composition pairing.

(3) There are natural isomorphisms

$$
\operatorname{Map}_{S p^{\Sigma}}(X \wedge K, Y) \cong \operatorname{Map}_{S p^{\Sigma}}\left(X, Y^{K}\right) \cong \operatorname{Map}_{S p^{\Sigma}}(X, Y)^{K}
$$

Proposition 1.3.1 says that certain functors are adjoints, whereas Proposition 1.3.2 says more; they are simplicial adjoints.

The category of symmetric spectra satisfies Quillen's axiom SM7 for simplicial model categories.

Definition 1.3.3. Let $f: U \rightarrow V$ and $g: X \rightarrow Y$ be maps of pointed simplicial sets. The pushout smash product $f \square g$ is the natural map on the pushout

$$
f \square g: V \wedge X \amalg_{U \wedge X} U \wedge Y \rightarrow V \wedge Y
$$

induced by the commutative square

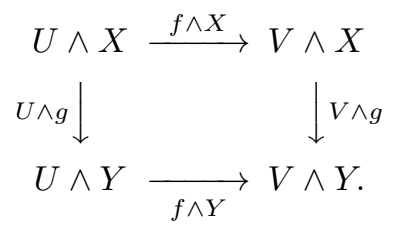

Let $f$ be a map of symmetric spectra and let $g$ be a map of pointed simplicial sets. The pushout smash product $f \square g$ is defined by prolongation, $(f \square g)_{n}=f_{n} \square g$.

Recall that a map of simplicial sets is a weak equivalence if its geometric realization is a homotopy equivalence of $\mathrm{CW}$-complexes. One of the basic properties of simplicial sets, proved in [Qui67, II.3], is:

Proposition 1.3.4. Let $f$ and $g$ be monomorphisms of pointed simplicial sets. Then $f \square g$ is a monomorphism, which is a weak equivalence if either $f$ or $g$ is a weak equivalence.

Prolongation gives a corollary for symmetric spectra. A map $f$ of symmetric spectra is a monomorphism if $f_{n}$ is a monomorphism of simplicial sets for each $n \geq 0$. 
Definition 1.3.5. A map $f$ of symmetric spectra is a level equivalence if $f_{n}$ is a weak equivalence of simplicial sets for each $n \geq 0$.

Corollary 1.3.6. Let $f$ be a monomorphism of symmetric spectra and let $g$ be a monomorphism of pointed simplicial sets. Then $f \square g$ is a monomorphism, which is a level equivalence if either $f$ is a level equivalence or $g$ is a weak equivalence.

By definition, a 0-simplex of $\operatorname{Map}_{S p^{\Sigma}}(X, Y)$ is a map $X \wedge \Delta[0]_{+} \rightarrow Y$, but $X \wedge \Delta[0]_{+} \cong X$ and so a 0-simplex of $\operatorname{Map}_{S p^{\Sigma}}(X, Y)$ is a map $X \rightarrow Y$. A 1-simplex of $\operatorname{Map}_{S p^{\Sigma}}(X, Y)$ is a simplicial homotopy $H: X \wedge \Delta[1]_{+} \rightarrow Y$ from $H \circ\left(X \wedge i_{0}\right)$ to $H \circ\left(X \wedge i_{1}\right)$ where $i_{0}$ and $i_{1}$ are the two inclusions $\Delta[0] \rightarrow \Delta[1]$. Simplicial homotopy generates an equivalence relation on $S p^{\Sigma}(X, Y)$ and the quotient is $\pi_{0} \operatorname{Map}_{S p^{\Sigma}}(X, Y)$. A map $f: X \rightarrow Y$ is a simplicial homotopy equivalence if it has a simplicial homotopy inverse, i.e., a map $g: Y \rightarrow X$ such that $g f$ is simplicially homotopic to the identity map on $X$ and $f g$ is simplicially homotopic to the identity map on $Y$. If $f$ is a simplicial homotopy equivalence of symmetric spectra, then each of the maps $f_{n}$ is a simplicial homotopy equivalence, and so each of the maps $f_{n}$ is a weak equivalence. Every simplicial homotopy equivalence is therefore a level equivalence. The converse is false; a map can be a level equivalence and NOT a simplicial homotopy equivalence.

1.4. Symmetric $\Omega$-spectra. The stable homotopy category can be defined using $\Omega$-spectra and level equivalences.

Definition 1.4.1. A Kan complex (see Example 3.2.6) is a simplicial set that satisfies the Kan extension condition. An $\Omega$-spectrum is a spectrum $X$ such that for each $n \geq 0$ the simplicial set $X_{n}$ is a Kan complex and the adjoint $X_{n} \rightarrow$ $\operatorname{Map}_{\mathcal{S}_{*}}\left(S^{1}, X_{n+1}\right)$ of the structure map $S^{1} \wedge X_{n} \rightarrow X_{n+1}$ is a weak equivalence of simplicial sets.

Let $\Omega S p^{\mathbb{N}} \subseteq S p^{\mathbb{N}}$ be the full subcategory of $\Omega$-spectra. The homotopy category $\operatorname{Ho}\left(\Omega S p^{\overline{\mathbb{N}}}\right)$ is obtained from $\Omega S p^{\mathbb{N}}$ by formally inverting the level equivalences. By the results in [BF78], the category $\operatorname{Ho}\left(\Omega S p^{\mathbb{N}}\right)$ is naturally equivalent to Boardman's stable homotopy category (or any other). Likewise, let $\Omega S p^{\Sigma} \subseteq S p^{\Sigma}$ be the full subcategory of symmetric $\Omega$-spectra (i.e., symmetric spectra $X$ for which $U X$ is an $\Omega$-spectrum). The homotopy category $\operatorname{Ho}\left(\Omega S p^{\Sigma}\right)$ is obtained from $\Omega S p^{\Sigma}$ by formally inverting the level equivalences. Since the forgetful functor $U: S p^{\Sigma} \rightarrow S p^{\mathbb{N}}$ preserves $\Omega$-spectra and level equivalences, it induces a functor $\mathrm{Ho}(U): \operatorname{Ho}\left(\Omega S p^{\Sigma}\right) \rightarrow \operatorname{Ho}\left(\Omega S p^{\mathbb{N}}\right)$. As a corollary of Theorem 4.2.5, the functor $\mathrm{Ho}(U)$ is a natural equivalence of categories. Thus the category $\operatorname{Ho}\left(\Omega S p^{\Sigma}\right)$ is naturally equivalent to Boardman's stable homotopy category. To describe an inverse of $\operatorname{Ho}(U)$, let $\Omega^{\infty}: S p^{\mathbb{N}} \rightarrow \mathcal{S}_{*}$ be the functor that takes a spectrum to the 0 -space of its associated $\Omega$-spectrum. For any spectrum $E \in S p^{\mathbb{N}}$, the symmetric spectrum $V E=\Omega^{\infty}(E \wedge S)$ is the value of the prolongation of the $\mathcal{S}_{*}$-functor $\Omega^{\infty}(E \wedge-)$ at the symmetric sphere spectrum $S$; the underlying sequence is $V E_{n}=\Omega^{\infty}\left(E \wedge S^{n}\right)$. The functor $V$ preserves $\Omega$-spectra, preserves level equivalences, and induces a functor $\mathrm{Ho}(V): \operatorname{Ho}\left(\Omega S p^{\mathbb{N}}\right) \rightarrow \mathrm{Ho}\left(\Omega S p^{\Sigma}\right)$ which is a natural inverse of $\mathrm{Ho}(U)$.

The category of symmetric $\Omega$-spectra has major defects. It is not closed under limits and colimits, or even under pushouts and pullbacks. The smash product, defined in Section 2, of symmetric $\Omega$-spectra is a symmetric spectrum but not an $\Omega$-spectrum, except in trivial cases. For these reasons it is better to work with 
the category of all symmetric spectra. But then the notion of level equivalence is no longer adequate; the stable homotopy category is a retract of the homotopy category obtained from $S p^{\Sigma}$ by formally inverting the level equivalences but many symmetric spectra are not level equivalent to an $\Omega$-spectrum. One must enlarge the class of equivalences. The stable equivalences of symmetric spectra are defined in Section 3.1. By Theorem 4.2.5, the homotopy category obtained from $S p^{\Sigma}$ by inverting the stable equivalences is naturally equivalent to the stable homotopy category.

\section{The SMASH PRODUCT OF SYMMETRIC SPECTRA}

In this section we construct the closed symmetric monoidal product on the category of symmetric spectra. A symmetric spectrum can be viewed as a module over the symmetric sphere spectrum $S$, and the symmetric sphere spectrum (unlike the ordinary sphere spectrum) is a commutative monoid in an appropriate category. The smash product of symmetric spectra is the tensor product over $S$.

The closed symmetric monoidal category of symmetric sequences is constructed in Section 2.1. A reformulation of the definition of a symmetric spectrum is given in Section 2.2 where we recall the definition of monoids and modules in a symmetric monoidal category. In Section 2.3 we see that there is no closed symmetric monoidal smash product on the category of (non-symmetric) spectra.

2.1. Symmetric sequences. Every symmetric spectrum has an underlying sequence $X_{0}, X_{1}, \ldots, X_{n}, \ldots$ of pointed simplicial sets with a basepoint preserving left action of $\Sigma_{n}$ on $X_{n}$; these are called symmetric sequences. In this section we define the closed symmetric monoidal category of symmetric sequences of pointed simplicial sets.

Definition 2.1.1. The category $\Sigma=\coprod_{n \geq 0} \Sigma_{n}$ has the finite sets $\bar{n}=\{1,2, \ldots, n\}$ for $n \geq 0(\overline{0}=\emptyset)$ as its objects and the automorphisms of the sets $\bar{n}$ as its maps. Let $\mathcal{C}$ be a category. A symmetric sequence of objects in $\mathcal{C}$ is a functor $\Sigma \rightarrow \mathcal{C}$, and the category of symmetric sequences of objects in $\mathcal{C}$ is the functor category $\mathcal{C}^{\Sigma}$.

A symmetric sequence $X \in \mathcal{S}_{*}^{\Sigma}$ is a sequence $X_{0}, X_{1}, \ldots, X_{n}, \ldots$ of pointed simplicial sets with a basepoint preserving left action of $\Sigma_{n}$ on $X_{n}$. The category $\mathrm{e}^{\Sigma}$ is a product category. In particular, $\mathcal{S}_{*}^{\Sigma}(X, Y)=\prod_{p} \mathcal{S}_{*}^{\Sigma_{p}}\left(X_{p}, Y_{p}\right)$.

Proposition 2.1.2. The category $\mathcal{S}_{*}^{\Sigma}$ of symmetric sequences in $\mathcal{S}_{*}$ is bicomplete.

Proof. The category $\mathcal{S}_{*}$ is bicomplete, so the functor category $\mathcal{S}_{*}^{\Sigma}$ is bicomplete.

Definition 2.1.3. The tensor product $X \otimes Y$ of the symmetric sequences $X, Y \in \mathcal{S}_{*}^{\Sigma}$ is the symmetric sequence

$$
(X \otimes Y)_{n}=\bigvee_{p+q=n}\left(\Sigma_{n}\right)_{+} \wedge_{\Sigma_{p} \times \Sigma_{q}}\left(X_{p} \wedge Y_{q}\right)
$$

The tensor product $f \otimes g: X \otimes Y \rightarrow X^{\prime} \otimes Y^{\prime}$ of the maps $f: X \rightarrow X^{\prime}$ and $g: Y \rightarrow Y^{\prime}$ in $\mathcal{S}_{*}^{\Sigma}$ is given by $(f \otimes g)(\alpha, x, y)=\left(\alpha, f_{p} x, g_{q} y\right)$ for $\alpha \in \Sigma_{p+q}, x \in X_{p}$ and $y \in Y_{q}$.

The tensor product of symmetric sequences has the universal property for "bilinear maps": 
Proposition 2.1.4. Let $X, Y, Z \in \mathcal{S}_{*}^{\Sigma}$ be symmetric sequences. Then there is a natural isomorphism

$$
\mathcal{S}_{*}^{\Sigma}(X \otimes Y, Z) \cong \prod_{p, q} \mathcal{S}_{*}^{\Sigma_{p} \times \Sigma_{q}}\left(X_{p} \wedge Y_{q}, Z_{p+q}\right) .
$$

The twist isomorphism $\tau: X \otimes Y \rightarrow Y \otimes X$ for $X, Y \in \mathcal{S}_{*}^{\Sigma}$ is the natural map given by $\tau(\alpha, x, y)=\left(\alpha \rho_{q, p}, y, x\right)$ for $\alpha \in \Sigma_{p+q}, x \in X_{p}$, and $y \in Y_{q}$, where $\rho_{q, p} \in \Sigma_{p+q}$ is the $(q, p)$-shuffle given by $\rho_{q, p}(i)=i+p$ for $1 \leq i \leq q$ and $\rho_{q, p}(i)=i-q$ for $q<i \leq p+q$. The map defined without the shuffle permutation is not a map of symmetric sequences.

Remark 2.1.5. There is another way of describing the tensor product and the twist isomorphism. The category $\Sigma$ is a skeleton of the category of finite sets and isomorphisms. Hence every symmetric sequence has an extension, which is unique up to isomorphism, to a functor on the category of all finite sets and isomorphisms. The tensor product of two such functors $X$ and $Y$ is the functor defined on a finite set $C$ as

$$
(X \otimes Y)(C)=\bigvee_{A \cup B=C, A \cap B=\emptyset} X(A) \wedge Y(B) .
$$

For an isomorphism $f: C \rightarrow D$ the map $(X \otimes Y)(f)$ is the coproduct of the isomorphisms $X(A) \wedge Y(B) \rightarrow X(f A) \wedge Y(f B)$. The twist isomorphism is the map that sends the summand $X(A) \wedge Y(B)$ of $(X \otimes Y)(C)$ to the summand $Y(B) \wedge X(A)$ of $(Y \otimes X)(C)$ by switching the factors.

Lemma 2.1.6. The tensor product $\otimes$ is a symmetric monoidal product on the category of symmetric sequences $\mathcal{S}_{*}^{\Sigma}$.

Proof. The unit of the tensor product is the symmetric sequence $\Sigma(\overline{0},-))_{+}=$ $\left(S^{0}, *, *, \ldots\right)$. The unit isomorphism is obvious. The associativity isomorphism is induced by the associativity isomorphism in $\mathcal{S}_{*}$ and the natural isomorphism

$$
((X \otimes Y) \otimes Z)_{n} \cong \bigvee_{p+q+r=n}\left(\Sigma_{n}\right)_{+} \wedge_{\Sigma_{p} \times \Sigma_{q} \times \Sigma_{r}}\left(X_{p} \wedge Y_{q} \wedge Z_{r}\right)
$$

The twist isomorphism is described in Remark 2.1.5. The coherence of the natural isomorphisms follows from coherence of the natural isomorphisms for the smash product in $\mathcal{S}_{*}$.

We now introduce several functors on the category of symmetric sequences.

Definition 2.1.7. The evaluation functor $\mathrm{Ev}_{n}: \mathcal{S}_{*}^{\Sigma} \rightarrow \mathcal{S}_{*}$ is given by $\operatorname{Ev}_{n} X=X_{n}$ and $\operatorname{Ev}_{n} f=f_{n}$. The free functor $G_{n}: \mathcal{S}_{*} \rightarrow \mathcal{S}_{*}^{\Sigma}$ is the left adjoint of the evaluation functor $\mathrm{Ev}_{n}$. The smash product $X \wedge K$ of $X \in \mathcal{S}_{*}^{\Sigma}$ and $K \in \mathcal{S}_{*}$ is the symmetric sequence $(X \wedge K)_{n}=X_{n} \wedge K$ with the diagonal action of $\Sigma_{n}$ that is trivial on $K$. The pointed simplicial set $\operatorname{Map}_{\mathcal{S}_{*}^{\Sigma}}(X, Y)$ of maps from $X$ to $Y$ is the pointed simplicial set $\mathcal{S}_{*}^{\Sigma}\left(X \wedge \Delta[-]_{+}, Y\right)$.

For each $n \geq 0$, the free symmetric sequence is $\Sigma[n]=\Sigma(\bar{n},-)$ and the free functor is $G_{n}=\Sigma[n]_{+} \wedge-: \mathcal{S}_{*} \rightarrow \mathcal{S}_{*}^{\Sigma}$. So, for a pointed simplicial set $K,\left(G_{n} K\right)_{n}=$ $\left(\Sigma_{n}\right)_{+} \wedge K$ and $\left(G_{n} K\right)_{k}=*$ for $k \neq n$. In particular, $G_{n} S^{0}=\Sigma[n]_{+}, G_{0} K=$ $(K, *, *, \ldots)$ and $G_{0} S^{0}$ is the unit of the tensor product $\otimes$.

We leave the proof of the following basic proposition to the reader. 
Proposition 2.1.8. There are natural isomorphisms:

(1) $G_{p} K \otimes G_{q} L \cong G_{p+q}(K \wedge L)$ for $K, L \in \mathcal{S}_{*}$.

(2) $X \otimes G_{0} K \cong X \wedge K$ for $K \in \mathcal{S}_{*}$ and $X \in \mathcal{S}_{*}^{\Sigma}$.

(3) $\operatorname{Map}_{\mathcal{S}_{*}^{\Sigma}}\left(G_{n} K, X\right) \cong \operatorname{Map}_{\mathcal{S}_{*}}\left(K, X_{n}\right)$ for $K \in \mathcal{S}_{*}$ and $X \in \mathcal{S}_{*}^{\Sigma}$.

(4) $\operatorname{Map}_{\mathcal{S}_{*}^{\Sigma}}(X \otimes Y, Z) \cong \prod_{p, q} \operatorname{Map}_{\Sigma_{p} \times \Sigma_{q}}\left(X_{p} \wedge Y_{q}, Z_{p+q}\right)$ for $X, Y, Z \in S p^{\Sigma}$.

A map $f$ of symmetric sequences is a level equivalence if each of the maps $f_{n}$ is a weak equivalence. Since $\mathcal{S}_{*}^{\Sigma}$ is a product category, a map $f$ of symmetric sequences is a monomorphism if and only if each of the maps $f_{n}$ is a monomorphism.

Proposition 2.1.9. Let $X$ be a symmetric sequence, let $f$ be a map of symmetric sequences and let $g$ be a map of pointed simplicial sets.

(1) $X \otimes(-)$ preserves colimits.

(2) If $f$ is a monomorphism, then $X \otimes f$ is a monomorphism.

(3) If $f$ is a level equivalence, then $X \otimes f$ is a level equivalence.

(4) If $g$ is a monomorphism, then $G_{n} g$ is a monomorphism for $n \geq 0$.

(5) If $g$ is a weak equivalence, then $G_{n} g$ is a level equivalence for $n \geq 0$.

Proof. Parts (1), (2) and (3) follow from the definition of $\otimes$ and the corresponding properties for the smash product of pointed simplicial sets. For Parts (4) and (5) use the isomorphism $G_{n} K=\Sigma[n]_{+} \wedge K$.

By part three of Proposition 2.1.8, $\operatorname{Map}\left(\Sigma[n]_{+}, X\right) \cong X_{n}$. As $n$ varies, $\Sigma[-]_{+}$is a functor $\Sigma^{\mathrm{op}} \rightarrow \mathcal{S}_{*}^{\Sigma}$, and for $X \in \mathcal{S}_{*}^{\Sigma}$, the symmetric sequence $\operatorname{Map}_{\mathcal{S}_{*}^{\Sigma}}\left(\Sigma[-]_{+}, X\right)$ is naturally isomorphic to $X$.

Definition 2.1.10. Let $X$ and $Y$ be symmetric sequences. The symmetric sequence of maps from $X$ to $Y$ is

$$
\operatorname{Hom}_{\Sigma}(X, Y)=\operatorname{Map}_{\mathcal{S}_{*}^{\Sigma}}\left(\Sigma[-]_{+} \otimes X, Y\right) .
$$

Theorem 2.1.11. The tensor product is a closed symmetric monoidal product on the category of symmetric sequences.

Proof. The tensor product is a symmetric monoidal product by Lemma 2.1.6. The product is closed if there is a natural isomorphism

$$
\mathcal{S}_{*}^{\Sigma}(X \otimes Y, Z) \cong \mathcal{S}_{*}^{\Sigma}\left(X, \operatorname{Hom}_{\Sigma}(Y, Z)\right)
$$

for symmetric sequences $X, Y$ and $Z$.

By Proposition 2.1.4, a map of symmetric sequences $f: X \otimes Y \rightarrow Z$ is a collection of $\Sigma_{p} \times \Sigma_{q}$-equivariant maps $f_{p, q}: X_{p} \wedge Y_{q} \rightarrow Z_{p+q}$. This is adjoint to a collection of $\Sigma_{p}$-equivariant maps $g_{p, q}: X_{p} \rightarrow \operatorname{Map}_{\Sigma_{q}}\left(Y_{q}, Z_{p+q}\right)$. So there is a natural isomorphism

$$
\mathcal{S}_{*}^{\Sigma}(X \otimes Y, Z) \cong \prod_{p} \mathcal{S}_{*}^{\Sigma_{p}}\left(X_{p}, \prod_{q} \operatorname{Map}_{\Sigma_{q}}\left(Y_{q}, Z_{p+q}\right)\right) .
$$

By Proposition 2.1.8, the functor sending $\bar{p}$ to $\prod_{q} \operatorname{Map}_{\Sigma_{q}}\left(Y_{q}, Z_{p+q}\right)$ is the functor sending $\bar{p}$ to $\operatorname{Map}\left(\Sigma[p]_{+} \otimes Y, Z\right)$ which by definition is $\operatorname{Hom}_{\Sigma}(Y, Z)$. Combining the isomorphisms gives the natural isomorphism that finishes the proof. 
2.2. Symmetric spectra. In this section we apply the language of "monoids" and "modules" in a symmetric monoidal category to the category of symmetric sequences. See [ML71, Bor94] for background on monoidal categories. In this language, the symmetric sequence of spheres $S=\left(S^{0}, S^{1}, \ldots, S^{n}, \ldots\right)$ is a commutative monoid in the category of symmetric sequences and a symmetric spectrum is a (left) $S$-module.

Consider the symmetric sphere spectrum $S$. By Proposition 2.1.4, the natural $\Sigma_{p} \times \Sigma_{q}$-equivariant maps $m_{p, q}: S^{p} \wedge S^{q} \rightarrow S^{p+q}$ give a pairing $m: S \otimes S \rightarrow S$. The adjoint $G_{0} S^{0} \rightarrow S$ of the identity map $S^{0} \rightarrow \mathrm{Ev}_{0} S=S^{0}$ is a two-sided unit of the pairing. The diagram of natural isomorphisms

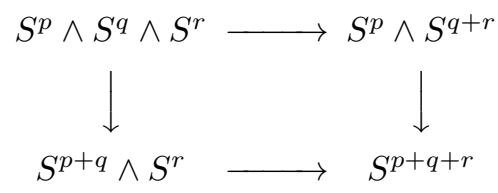

commutes, showing that $m$ is an associative pairing of symmetric sequences.

In the language of monoidal categories, $S$ is a monoid in the category of symmetric sequences and a symmetric spectrum is a left $S$-module.

Proposition 2.2.1. The category of symmetric spectra is naturally equivalent to the category of left $S$-modules.

Proof. A pairing $m: S \otimes X \rightarrow X$ is the same as a collection of $\Sigma_{p} \times \Sigma_{q}$-equivariant maps $m_{p, q}: S^{p} \wedge X_{q} \rightarrow X_{p+q}$. If $X$ is a left $S$-module, there is a spectrum for which $X$ is the underlying symmetric sequence and the structure maps are the maps $\sigma=m_{1, n}: S^{1} \wedge X_{n} \rightarrow X_{n+1}$. The compositions $\sigma^{p}$ are the $\Sigma_{p} \times \Sigma_{q}$-equivariant maps $m_{p, q}$. Conversely, for $X$ a symmetric spectrum, the map of symmetric sequences $m: S \otimes X \rightarrow X$ corresponding to the collection of $\Sigma_{p} \times \Sigma_{q}$-equivariant maps $m_{p, q}=$ $\sigma^{p}: S^{p} \wedge X_{q} \rightarrow X_{p+q}$, where $\sigma^{0}$ is the natural isomorphism $S^{0} \wedge X_{n} \rightarrow X_{n}$, makes $X$ a left $S$-module. These are inverse constructions and give a natural equivalence of categories.

Moreover, $S$ is a commutative monoid, i.e., $m=m \circ \tau$, where $\tau$ is the twist isomorphism. To see this, one can use either the definition of the twist isomorphism or the description given in Remark 2.1.5. Then, as is the case for commutative monoids in the category of sets and for commutative monoids in the category of abelian groups (i.e., commutative rings), there is a tensor product $\otimes_{S}$, having $S$ as the unit. This gives a symmetric monoidal product on the category of $S$-modules. The smash product $X \wedge Y$ of $X, Y \in S p^{\Sigma}$ is the symmetric spectrum $X \otimes_{S} Y$.

The smash product on the category of symmetric spectra is a special case of the following lemma.

Lemma 2.2.2. Let $\mathrm{C}$ be a symmetric monoidal category that is cocomplete and let $R$ be a commutative monoid in $\mathcal{C}$ such that the functor $R \otimes(-): \mathcal{C} \rightarrow \mathcal{C}$ preserves coequalizers. Then there is a symmetric monoidal product $\otimes_{R}$ on the category of $R$-modules with $R$ as the unit.

We leave the proof of this lemma to the reader; the main point is the following definition.

Definition 2.2.3. The smash product $X \wedge Y$ of symmetric spectra $X$ and $Y$ is the symmetric spectrum $X \otimes_{S} Y$. The tensor product $X \otimes_{S} Y$ is the colimit in 
symmetric sequences of the diagram

$$
X \otimes S \otimes Y \underset{1 \otimes m}{\stackrel{m \otimes 1}{\longrightarrow}} X \otimes Y .
$$

For $X$ a left $S$-module the composition $X \otimes S \stackrel{\tau}{\rightarrow} S \otimes X \stackrel{\alpha}{\rightarrow} X$ is the right action of $S$; since $S$ is commutative, the two actions commute and $X$ is an $(S, S)$-bimodule. Hence, the tensor product $X \otimes_{S} Y$ is a left $S$-module.

Apply Lemma 2.2.2 to the commutative monoid $S$ in the bicomplete category of symmetric sequences $\mathcal{S}_{*}^{\Sigma}$ to obtain the following corollary.

Corollary 2.2.4. The smash product $X \wedge Y$ is a symmetric monoidal product on the category of symmetric spectra.

Next, some important functors on the category of symmetric spectra.

Definition 2.2.5. The functor $S \otimes(-): \mathcal{S}_{*}^{\Sigma} \rightarrow S p^{\Sigma}$ gives the free $S$-module $S \otimes X$ generated by the symmetric sequence $X$. For each $n \geq 0$, the evaluation functor $\mathrm{Ev}_{n}: S p^{\Sigma} \rightarrow \mathcal{S}_{*}$ is given by $\operatorname{Ev}_{n} X=X_{n}$ and $\operatorname{Ev}_{n} f=f_{n}$. The free functor $F_{n}: \mathcal{S}_{*} \rightarrow S p^{\Sigma}$ is the left adjoint of the evaluation functor $\operatorname{Ev}_{n}$. The functor $R_{n}: \mathcal{S}_{*} \rightarrow S p^{\Sigma}$ is the right adjoint of the evaluation functor $\operatorname{Ev}_{n}: S p^{\Sigma} \rightarrow \mathcal{S}_{*}$.

The functor $S \otimes(-)$ is left adjoint to the forgetful functor $S p^{\Sigma} \rightarrow \mathcal{S}_{*}^{\Sigma}$. The free functor $F_{n}$ is the composition $S \otimes G_{n}$ of the left adjoints $G_{n}: \mathcal{S}_{*} \rightarrow \mathcal{S}_{*}^{\Sigma}$ (Definition 2.1.7) and $S \otimes(-): \mathcal{S}_{*}^{\Sigma} \rightarrow S p^{\Sigma}$. Thus, for $X \in S p^{\Sigma}$ and $K \in \mathcal{S}_{*}$, the left $S$-module $X \wedge F_{n} K$ is naturally isomorphic to the left $S$-module $X \otimes G_{n} K$. In particular, $X \wedge F_{0} K$ is naturally isomorphic to the symmetric spectrum $X \wedge K$ defined by prolongation in Section 1.3. Furthermore $F_{0} K=S \wedge K$ is the symmetric suspension spectrum $\Sigma^{\infty} K$ of $K$, and $F_{0} S^{0}$ is the symmetric sphere spectrum $S$. For a pointed simplicial set $K, R_{n} K$ is the symmetric sequence $\operatorname{Hom}_{\mathcal{S}_{*}^{\Sigma}}\left(S, K^{\Sigma(-, \bar{n})_{+}}\right)$, which is a left $S$-module since $S$ is a right $S$-module.

We leave the proof of the following proposition to the reader.

Proposition 2.2.6. There are natural isomorphisms:

(1) $F_{m}(K) \wedge F_{n}(L) \cong F_{m+n}(K \wedge L)$ for $K, L \in \mathcal{S}_{*}$.

(2) $\operatorname{Map}_{S p^{\Sigma}}(S \otimes X, Y) \cong \operatorname{Map}_{\mathcal{S}_{*}^{\Sigma}}(X, Y)$ for $X \in \mathcal{S}_{*}^{\Sigma}$ and $Y \in S p^{\Sigma}$.

(3) $\operatorname{Map}_{S p^{\Sigma}}\left(F_{n} K, X\right) \cong \operatorname{Map}_{\mathcal{S}_{*}}\left(K, \operatorname{Ev}_{n} X\right)$ for $K \in \mathcal{S}_{*}$ and $X \in S p^{\Sigma}$.

Proposition 2.2.7. Let $f$ be a map of pointed simplicial sets.

(1) $F_{n}: \mathcal{S}_{*} \rightarrow S p^{\Sigma}$ preserves colimits.

(2) If $f$ is a monomorphism, then $F_{n} f$ is a monomorphism.

(3) If $f$ is a weak equivalence, then $F_{n} f$ is a level equivalence.

Proof. Use the isomorphism $F_{n} f=S \otimes G_{n} f$ and Proposition 2.1.9.

The internal Hom on the category of symmetric spectra is a special case of the following lemma.

Lemma 2.2.8. Let $\mathcal{C}$ be a closed symmetric monoidal category that is bicomplete and let $R$ be a commutative monoid in $\mathcal{C}$. Then there is a function $R$-module $\operatorname{Hom}_{R}(M, N)$, natural for $M, N \in \mathcal{C}$, such that the functor $(-) \otimes_{R} M$ is left adjoint to the functor $\operatorname{Hom}_{R}(M,-)$. 
Again, we leave the proof of this lemma to the reader, but the main definition follows.

Definition 2.2.9. Let $X$ and $Y$ be symmetric spectra. The function spectrum $\operatorname{Hom}_{S}(X, Y)$ is the limit of the diagram in $S p^{\Sigma}$

$$
\operatorname{Hom}_{\Sigma}(X, Y) \underset{m_{*}}{\stackrel{m^{*}}{\longrightarrow}} \operatorname{Hom}_{\Sigma}(S \otimes X, Y) .
$$

Combining Lemmas 2.2.2 and 2.2.8:

Theorem 2.2.10. The smash product is a closed symmetric monoidal product on the category of symmetric spectra. In particular, there is a natural adjunction isomorphism

$$
S p^{\Sigma}(X \wedge Y, Z) \cong S p^{\Sigma}\left(X, \operatorname{Hom}_{S}(Y, Z)\right) .
$$

Proof. The smash product $\wedge$ is a symmetric monoidal product by Corollary 2.2.4. The adjunction isomorphism follows from Lemma 2.2.8.

The adjunction is also a simplicial adjunction and an internal adjunction.

Corollary 2.2.11. There are natural isomorphisms

$$
\operatorname{Map}_{S p^{\Sigma}}(X \wedge Y, Z) \cong \operatorname{Map}_{S p^{\Sigma}}\left(X, \operatorname{Hom}_{S}(Y, Z)\right)
$$

and

$$
\operatorname{Hom}_{S}(X \wedge Y, Z) \cong \operatorname{Hom}_{S}\left(X, \operatorname{Hom}_{S}(Y, Z)\right) .
$$

Remark 2.2.12. We use Proposition 2.2.6 to give another description of the function spectrum $\operatorname{Hom}_{S}(X, Y)$. For a symmetric spectrum $X$, the pointed simplicial set of maps $\operatorname{Map}_{S p^{\Sigma}}\left(F_{n} S^{0}, X\right)$ is naturally isomorphic to $\operatorname{Map}_{\mathcal{S}_{*}}\left(S^{0}, \operatorname{Ev}_{n} X\right)=X_{n}$. The symmetric spectrum $F_{n} S^{0}$ is the $S$-module $S \otimes \Sigma[n]_{+}$and as $n$ varies, $S \otimes$ $\Sigma[-]_{+}$is a functor $\Sigma^{\mathrm{op}} \rightarrow S p^{\Sigma}$. The symmetric sequence $\operatorname{Map}_{S p^{\Sigma}}\left(S \otimes \Sigma[-]_{+}, X\right)$ is the underlying symmetric sequence of $X$. In particular, the natural isomorphism $X_{n}=\operatorname{Map}_{S p^{\Sigma}}\left(F_{n} S^{0}, X\right)$ is $\Sigma_{n}$-equivariant. Applying this to $\operatorname{Hom}_{S}(X, Y)$ and using Corollary 2.2.11, we find that the underlying symmetric sequence of $\operatorname{Hom}_{S}(X, Y)$ is the symmetric sequence $\operatorname{Map}_{S p^{\Sigma}}\left(X \wedge\left(S \otimes \Sigma[-]_{+}\right), Y\right)$.

We must also describe the structure maps of $X$ from this point of view. Recall that $\operatorname{Map}_{S p^{\Sigma}}\left(F_{n} S^{0}, X\right)=X_{n}, \operatorname{Map}_{S p^{\Sigma}}\left(F_{n} S^{1}, X\right)=\operatorname{Map}_{\mathcal{S}_{*}}\left(S^{1}, X_{n}\right)$. Let $\lambda: F_{1} S^{1} \rightarrow F_{0} S^{0}$ be the adjoint of the identity map $S^{1} \rightarrow \operatorname{Ev}_{1} F_{0} S^{0}=S^{1}$. The induced map $\operatorname{Map}_{S p^{\Sigma}}(\lambda, X): X_{0} \rightarrow \operatorname{Map}_{\mathcal{S}_{*}}\left(S^{1}, X_{1}\right)$ is adjoint to the structure map $S^{1} \wedge X_{0} \rightarrow X_{1}$. The map

$$
\lambda \wedge F_{n} S^{0}: F_{1} S^{1} \wedge F_{n} S^{0}=F_{n+1} S^{1} \rightarrow F_{0} S^{0} \wedge F_{n} S^{0}=F_{n} S^{0}
$$

is $\Sigma_{1} \times \Sigma_{n}$-equivariant; the induced map

$$
\operatorname{Map}_{S p^{\Sigma}}\left(\lambda \wedge F_{n} S^{0}, X\right): X_{n} \rightarrow \operatorname{Map}_{\mathcal{S}_{*}}\left(S^{1}, X_{n+1}\right)
$$

is $\Sigma_{1} \times \Sigma_{n}$-equivariant and is adjoint to the structure map $\sigma: S^{1} \wedge X_{n} \rightarrow X_{n+1}$. In order to apply this to $\operatorname{Hom}_{S}(X, Y)$, use Proposition 2.2.6 and Corollary 2.2.11 to find a natural isomorphism

$$
\operatorname{Map}_{S p^{\Sigma}}\left(X \wedge F_{n+1} S^{1}, Y\right) \cong \operatorname{Map}_{\mathcal{S}_{*}}\left(S^{1}, \operatorname{Map}_{S p^{\Sigma}}\left(X \wedge F_{n+1} S^{0}, Y\right)\right) .
$$


Using this natural isomorphism, we find that the structure maps of $\operatorname{Hom}_{S}(X, Y)$ are the adjoints of the maps

$$
\operatorname{Map}_{S p^{\Sigma}}\left(X \wedge F_{n} S^{0}, Y\right) \rightarrow \operatorname{Map}_{S p^{\Sigma}}\left(X \wedge F_{n+1} S^{1}, Y\right)
$$

induced by $\lambda \wedge F_{n} S^{0}$.

For example, $\operatorname{Hom}_{S}\left(F_{k} S^{0}, X\right)$ is the $k$-shifted spectrum; its underlying symmetric sequence is the sequence of pointed simplicial sets

$$
X_{k}, X_{1+k}, \ldots, X_{n+k}, \ldots
$$

with $\Sigma_{n}$ acting on $X_{n+k}$ by restricting the action of $\Sigma_{n+k}$ to the copy of $\Sigma_{n}$ that permutes the first $n$ elements of $\overline{n+k}$. The structure maps of the $k$-shifted spectrum are the structure maps $\sigma: S^{1} \wedge X_{n+k} \rightarrow X_{n+k+1}$ of $X$. More generally, $\operatorname{Hom}_{S}\left(F_{k} K, X\right)$ is the $k$-shifted spectrum of $X^{K}$.

2.3. The ordinary category of spectra. An approach similar to the last two sections can be used to describe (non-symmetric) spectra as modules over the sphere spectrum in a symmetric monoidal category. But in this case the sphere spectrum is not a commutative monoid, which is why there is no closed symmetric monoidal smash product of spectra.

Definition 2.3.1. The category $\mathbb{N}$ is the category with the non-negative integers as its objects and with the identity maps of the objects as its only maps. The category of sequences $\mathcal{S}_{*}^{\mathbb{N}}$ is the category of functors from $\mathbb{N}$ to $\mathcal{S}_{*}$. An object of $\mathcal{S}_{*}^{\mathbb{N}}$ is a sequence $X_{0}, X_{1}, \ldots, X_{n}, \ldots$ of pointed simplicial sets and a map $f: X \rightarrow Y$ is a sequence of pointed simplicial maps $f_{n}: X_{n} \rightarrow Y_{n}$.

Definition 2.3.2. The graded smash product of sequences $X$ and $Y$ is the sequence $X \otimes Y$ given in degree $n$ by

$$
(X \otimes Y)_{n}=\bigvee_{p+q=n} X_{p} \wedge Y_{q}
$$

Lemma 2.3.3. The category of sequences is a bicomplete category and the graded smash product is a symmetric monoidal product on $\mathcal{S}_{*}^{\mathbb{N}}$.

Proposition 2.3.4. The sequence $S$ whose nth level is $S^{n}$ is a monoid in the category of sequences. The category of left $S$-modules is isomorphic to the ordinary category of spectra, $S p^{\mathbb{N}}$.

The twist map on $S^{1} \wedge S^{1}$ is not the identity map and thus $S$ is not a commutative monoid in $\mathcal{S}_{*}^{\mathbb{N}}$. In fact, $S$ is a free monoid (Section 4.3). Therefore the approach taken in Section 2.2 does not provide a closed symmetric monoidal smash product on the ordinary category of spectra.

\section{StABle homotopy THEORY OF SYMMETRIC SPECTRA}

To use symmetric spectra for the study of stable homotopy theory, one should have a stable model category of symmetric spectra such that the category obtained by inverting the stable equivalences is naturally equivalent to Boardman's stable homotopy category of spectra (or to any other known to be equivalent to Boardman's). In this section we define the stable model category of symmetric spectra. In Section 4 we show that it is Quillen equivalent to the stable model category of spectra discussed in [BF78]. 
In Section 3.1 we define the class of stable equivalences of symmetric spectra and discuss its non-trivial relationship to the class of stable equivalences of (nonsymmetric) spectra. In Section 3.2 we recall the axioms and basic theory of model categories. In Section 3.3 we discuss the level structure in $S p^{\Sigma}$, and in Section 3.4 we define the stable model structure on the category of symmetric spectra which has the stable equivalences as the class of weak equivalences. The rest of the section is devoted to checking that the stable model structure satisfies the axioms of a model category.

3.1. Stable equivalence. One's first inclination is to define stable equivalence using the forgetful functor $U: S p^{\Sigma} \rightarrow S p^{\mathbb{N}}$; one would like a map $f$ of symmetric spectra to be a stable equivalence if the underlying map $U f$ of spectra is a stable equivalence, i.e., if $U f$ induces an isomorphism of stable homotopy groups. The reader is warned: THIS WILL NOT WORK. Instead, stable equivalence is defined using cohomology; a map $f$ of symmetric spectra is a stable equivalence if the induced map $E^{*} f$ of cohomology groups is an isomorphism for every generalized cohomology theory $E$. The two alternatives, using stable homotopy groups or using cohomology groups, give equivalent definitions on the category of (non-symmetric) spectra but not on the category of symmetric spectra.

It would be nice if the 0th cohomology group of the symmetric spectrum $X$ with coefficients in the symmetric $\Omega$-spectrum $E$ could be defined as $\pi_{0} \operatorname{Map}_{S p^{\Sigma}}(X, E)$, the set of simplicial homotopy classes of maps from $X$ to $E$. But, even though the contravariant functor $E^{0}=\pi_{0} \operatorname{Map}_{S p^{\Sigma}}(-, E)$ takes simplicial homotopy equivalences to isomorphisms, $E^{0}$ may not take level equivalences to isomorphisms. This is a common occurrence in simplicial categories, but is a problem as every level equivalence should induce an isomorphism of cohomology groups; a level equivalence certainly induces an isomorphism of stable homotopy groups. We introduce injective spectra as a class of spectra $E$ for which the functor $E^{0}$ behaves correctly.

Definition 3.1.1. An injective spectrum is a symmetric spectrum $E$ that has the extension property with respect to every monomorphism $f$ of symmetric spectra that is a level equivalence. That is, for every diagram in $S p^{\Sigma}$

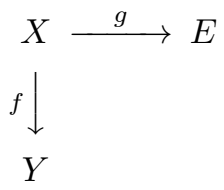

where $f$ is a monomorphism and a level equivalence there is a map $h: Y \rightarrow E$ such that $g=h f$.

Some examples of injective spectra follow. Recall that $R_{n}: \mathcal{S}_{*} \rightarrow S p^{\Sigma}$ is the right adjoint of the evaluation functor $\mathrm{Ev}_{n}: S p^{\Sigma} \rightarrow \mathcal{S}_{*}$. Also recall that a Kan complex has the extension property with respect to every map of pointed simplicial sets that is a monomorphism and a weak equivalence.

Lemma 3.1.2. If the pointed simplicial set $K$ is a Kan complex, then $R_{n} K$ is an injective spectrum. If $X$ is a symmetric sequence and $E$ is an injective spectrum, then $\operatorname{Hom}_{S}(S \otimes X, E)$ is an injective spectrum.

Proof. Since $\mathrm{Ev}_{n}$ is left adjoint to $R_{n}$, the spectrum $R_{n} K$ has the extension property with respect to the monomorphism and level equivalence $f$ if and only if $K$ has 
the extension property with respect to the monomorphism and weak equivalence $\operatorname{Ev}_{n} f$. Since $K$ is a Kan complex, it does have the extension property with respect to $\operatorname{Ev}_{n} f$. Hence $R_{n} K$ is injective.

Since the functor $(S \otimes X) \wedge(-)$ is the left adjoint of $\operatorname{Hom}_{S}(S \otimes X,-)$, the spectrum $\operatorname{Hom}_{S}(S \otimes X, E)$ has the extension property with respect to the monomorphism and level equivalence $f$ if and only if $E$ has the extension property with respect to the map $(S \otimes X) \wedge f$. There is a natural isomorphism of maps of symmetric sequences $(S \otimes X) \wedge f \cong X \otimes f$. Since $f$ is a monomorphism and a level equivalence, $X \otimes f$ is a monomorphism and a level equivalence by Proposition 2.1.9. Thus $(S \otimes X) \wedge f$ is also a monomorphism and level equivalence of symmetric spectra. $\operatorname{So}_{\operatorname{Hom}}(S \otimes X, E)$ is injective.

In fact, injective spectra are the fibrant objects of a model structure on $S p^{\Sigma}$ for which every object is cofibrant (Section 5.1). In particular, as we will see in Corollary 5.1.3, there are enough injectives; every symmetric spectrum embeds in an injective spectrum by a map that is a level equivalence.

Definition 3.1.3. A map $f: X \rightarrow Y$ of symmetric spectra is a stable equivalence if $E^{0} f: E^{0} Y \rightarrow E^{0} X$ is an isomorphism for every injective $\Omega$-spectrum $E$.

There are two other ways to define stable equivalence.

Proposition 3.1.4. Let $f: X \rightarrow Y$ be a map of symmetric spectra. The following conditions are equivalent:

- $E^{0} f$ is an isomorphism for every injective $\Omega$-spectrum $E$;

- $\operatorname{Map}_{S p^{\Sigma}}(f, E)$ is a weak equivalence for every injective $\Omega$-spectrum $E$;

- $\operatorname{Hom}_{S}(f, E)$ is a level equivalence for every injective $\Omega$-spectrum $E$.

Proof. Let $K$ be a pointed simplicial set and let $E$ be a symmetric $\Omega$-spectrum. The adjoints of the structure maps of $E$ are weak equivalences of Kan complexes. From Remark 2.2.12, for $k, n \geq 0 \operatorname{Ev}_{k} \operatorname{Hom}_{S}\left(F_{n} K, E\right)=E_{n+k}^{K}$. The adjoints of the structure maps of $\operatorname{Hom}_{S}\left(F_{n} K, E\right)$ are the weak equivalences of Kan complexes $E_{n+k}^{K} \rightarrow E_{n+k+1}^{S^{1} \wedge K}$ induced by the adjoints of the structure maps of $E$. Therefore, $\operatorname{Hom}_{S}\left(F_{n} K, E\right)$ is an $\Omega$-spectrum.

Now let $E$ be an injective $\Omega$-spectrum. Using the natural isomorphism $F_{n} K \cong$ $S \otimes G_{n} K$ and Lemma 3.1.2, $\operatorname{Hom}_{S}\left(F_{n} K, E\right)$ is an injective spectrum. By the preceding paragraph, $\operatorname{Hom}_{S}\left(F_{n} K, E\right)$ is an $\Omega$-spectrum. Hence $E^{S^{n}}=\operatorname{Hom}_{S}\left(F_{0}\left(S^{n}\right), E\right)$ and the $k$-shifted spectrum $\operatorname{Hom}_{S}\left(F_{k} S^{0}, E\right)$ are injective $\Omega$-spectra. Given a stable equivalence $f: X \rightarrow Y$, we want to show that $\operatorname{Map}_{S p^{\Sigma}}(f, E)$ is a weak equivalence. Since

$$
\pi_{n} \operatorname{Map}_{S p^{\Sigma}}(f, E)=\pi_{0} \operatorname{Map}_{S p^{\Sigma}}\left(f, E^{S^{n}}\right)
$$

and the simplicial sets $\operatorname{Map}_{S p^{\Sigma}}(Y, E)$ and $\operatorname{Map}_{S p^{\Sigma}}(X, E)$ are Kan complexes by Lemma 3.1.5, $\operatorname{Map}_{S p^{\Sigma}}(f, E)$ is a weak equivalence on the basepoint components. We must extend this to all components. To do so, note that $\operatorname{Map}_{S p^{\Sigma}}(f, E)^{S^{1}}$ is a weak equivalence for any injective $\Omega$-spectrum $E$, since the loop space only depends on the basepoint component. Consider the commutative diagram

$$
\begin{aligned}
\operatorname{Map}_{S p^{\Sigma}}(Y, E) & \longrightarrow \operatorname{Map}_{S p^{\Sigma}}\left(Y, \operatorname{Hom}_{S}\left(F_{1} S^{0}, E\right)\right)^{S^{1}} \\
\operatorname{Map}_{S p^{\Sigma}}(f, E) & \downarrow \\
\operatorname{Map}_{S p^{\Sigma}}(X, E) & \longrightarrow \operatorname{Map}_{S p^{\Sigma}}\left(X, \operatorname{Hom}_{S}\left(F_{1} S^{0}, E\right)\right)^{S^{1}}
\end{aligned}
$$


where the horizontal maps are induced by the map $E \rightarrow \operatorname{Hom}_{S}\left(F_{1} S^{0}, E\right)^{S^{1}}$ adjoint to the structure map of $E$. Since $E$ is an injective $\Omega$-spectrum, this map is a level equivalence of injective spectra. By Lemma 3.1.6, it is a simplicial homotopy equivalence. Hence the horizontal maps in the diagram above are weak equivalences. Since the right-hand vertical map is a weak equivalence, so is the left-hand vertical map $\operatorname{Map}_{S p^{\Sigma}}(f, E)$. Thus the first two conditions in the proposition are equivalent. Since $\operatorname{Ev}_{k} \operatorname{Hom}_{S}(f, E)=\operatorname{Map}_{S p^{\Sigma}}\left(f, \operatorname{Hom}_{S}\left(F_{k} S^{0}, E\right)\right)$, the second two conditions are equivalent.

Lemma 3.1.5. Suppose $X$ is a symmetric spectrum and $E$ is an injective spectrum. Then the pointed simplicial set $\operatorname{Map}_{S p^{\Sigma}}(X, E)$ is a Kan complex. In particular, each pointed simplicial set $E_{n}$ is a Kan complex.

Proof. Suppose that $f: K \rightarrow L$ is a monomorphism and weak equivalence of simplicial sets. We must show that $\operatorname{Map}_{S p^{\Sigma}}(X, E)$ has the extension property with respect to $f$. By adjointness, this is equivalent to showing that $E$ has the extension property with respect to $X \wedge f$. But $X \wedge f$ is a monomorphism and level equivalence by Corollary 1.3.6 applied to the monomorphism $* \rightarrow X$ and $f$, so $E$ does have the required extension property.

The basic properties of injective spectra which are needed in the rest of this section are stated in the following lemma.

Lemma 3.1.6. Let $f: X \rightarrow Y$ be a map of symmetric spectra.

(1) If $E \in S p^{\Sigma}$ is an injective spectrum and $f$ is a level equivalence, then $E^{0} f$ is an isomorphism of sets.

(2) If $f: X \rightarrow Y$ is a map of injective spectra, $f$ is a level equivalence if and only if $f$ is a simplicial homotopy equivalence.

The proof uses the following construction.

Construction 3.1.7 (Mapping cylinder construction). The mapping cylinder construction for maps of symmetric spectra is the prolongation of the reduced mapping cylinder construction for maps of pointed simplicial sets. Let $i_{0}$ and $i_{1}$ be the two inclusions $\Delta[0] \rightarrow \Delta[1]$. The cylinder spectrum $M f$ of a map $f: X \rightarrow Y \in S p^{\Sigma}$ is the corner of the pushout square

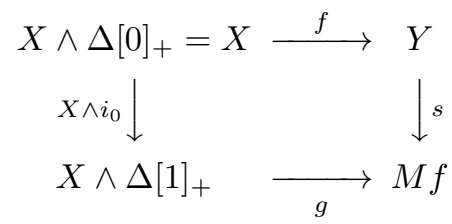

Let $i=g \circ\left(X \wedge i_{1}\right): X \rightarrow M f$. Let $r: M f \rightarrow Y$ be the map on the pushout induced by the identity map on $Y$ and the composition $X \wedge \Delta[1]_{+} \rightarrow X \rightarrow Y$. Then $f=r i, i$ is a monomorphism, $r s=i d_{Y}$, and there is a simplicial homotopy from $s r$ to the identity map of $M f$.

Proof of Lemma 3.1.6. For part one of the lemma, let $f: X \rightarrow Y$ be a level equivalence and let $M f$ be the mapping cylinder of $f$. As above $f=r i, i: X \rightarrow M f$ is a monomorphism, and $r: M f \rightarrow Y$ is a simplicial homotopy equivalence. Then $E^{0} r$ is an isomorphism and, if $E^{0} i$ is an isomorphism, the composition $E^{0} f$ is an isomorphism. The map $i$ is a monomorphism which, by the 2-out-of-3 property, is 
a level equivalence. By the extension property of $E$ with respect to $i$, the map $E^{0} i$ is surjective. The inclusion of the boundary $j: \partial \Delta[1] \rightarrow \Delta[1]$ is a monomorphism. By Corollary 1.3.6, the map

$$
i \square j: X \wedge \Delta[1]_{+} \amalg_{X \wedge \partial \Delta[1]_{+}} M f \wedge \partial \Delta[1]_{+} \rightarrow M f \wedge \Delta[1]_{+}
$$

is a monomorphism and a level equivalence. The extension property of $E$ with respect to $i \square j$ implies that if $g, h: M f \rightarrow E$ are maps such that $g i$ and $h i$ are simplicially homotopic, then $g$ and $h$ are simplicially homotopic. So $E^{0} i$ is a monomorphism and hence $E^{0} i$ is an isomorphism.

For the second part of the lemma, if $f$ is a simplicial homotopy equivalence, each $f_{n}$ is a simplicial homotopy equivalence of simplicial sets and so each $f_{n}$ is a weak equivalence. Conversely, suppose $f: X \rightarrow Y$ is a level equivalence of injective spectra. By part one, $X^{0} f: X^{0} Y \rightarrow X^{0} X$ is an isomorphism. The inverse image of the equivalence class of the identity map $X \rightarrow X$ is an equivalence class of maps $Y \rightarrow X$. Since $Y$ is injective, $Y^{0} f: Y^{0} Y \rightarrow Y^{0} X$ is an isomorphism. Hence each map in the equivalence class is a simplicial homotopy inverse of $f$.

Restricting part one of Lemma 3.1.6 to injective $\Omega$-spectra gives:

Corollary 3.1.8. Every level equivalence of symmetric spectra is a stable equivalence.

Next recall the definition of stable homotopy equivalence in the category of (nonsymmetric) spectra $S p^{\mathbb{N}}$.

Definition 3.1.9. For each integer $k$ the $k$ th homotopy group of the spectrum (or symmetric spectrum) $X$ is the colimit

$$
\pi_{k} X=\operatorname{colim}_{n} \pi_{k+n} X_{n}
$$

of the directed system given by the compositions

$$
\pi_{k+n} X_{n} \stackrel{E}{\longrightarrow} \pi_{k+n+1}\left(S^{1} \wedge X_{n}\right) \stackrel{\pi_{k+n+1} \sigma}{\longrightarrow} \pi_{k+n+1} X_{n+1}
$$

of the suspension homomorphism and the map induced by $\sigma$.

A map of spectra $f \in S p^{\mathbb{N}}$ is a stable homotopy equivalence if $\pi_{*} f$ is an isomorphism. For example, every level equivalence in $S p^{\mathbb{N}}$ is a stable homotopy equivalence as it induces an isomorphism of homotopy groups. We do not define stable equivalence of symmetric spectra in this way; as the following example shows, a stable equivalence of symmetric spectra need not induce an isomorphism of homotopy groups.

Example 3.1.10. The map $\lambda: F_{1} S^{1} \rightarrow F_{0} S^{0}$ (see 2.2.12) is the adjoint of the identity map $S^{1} \rightarrow \operatorname{Ev}_{1} S=S^{1}$. The $n$th space of $F_{1} S^{1}$ is $\left(\Sigma_{n}\right)_{+} \wedge_{\Sigma_{n-1}} S^{n}$, which is a wedge of $n$ copies of $S^{n}$. One can calculate that $\pi_{0} F_{1} S^{1}$ is an infinite direct sum of copies of the integers $\mathbb{Z}$, whereas $\pi_{0} F_{0} S^{0}$ is $\mathbb{Z}$. So $\pi_{*} \lambda$ is not an isomorphism of homotopy groups and thus $U \lambda$ is not a stable homotopy equivalence of (non-symmetric) spectra. But $\lambda$ is a stable equivalence of symmetric spectra. For a symmetric $\Omega$-spectrum $E, \operatorname{Map}_{S p^{\Sigma}}\left(F_{1} S^{1}, E\right)=\operatorname{Map}_{\mathcal{S}_{*}}\left(S^{1}, E_{1}\right)$, $\operatorname{Map}_{S p^{\Sigma}}\left(F_{0} S^{0}, E\right)=E_{0}$, and the induced map $\operatorname{Map}_{S p^{\Sigma}}(\lambda, E)=E_{0} \rightarrow E_{1}^{S^{1}}$ is adjoint to the structure map $S^{1} \wedge E_{0} \rightarrow E_{1}$. So $\operatorname{Map}_{S p^{\Sigma}}(\lambda, E)$ is a weak equivalence for every $\Omega$-spectrum $E$, including the injective ones, and so $\lambda$ is a stable equivalence. By the same argument, the maps $\lambda \wedge F_{n} S^{0}$ are stable equivalences. 
The forgetful functor $U: S p^{\Sigma} \rightarrow S p^{\mathbb{N}}$ does not preserve stable equivalences. On the other hand, the functor $U$ does reflect stable equivalences.

Theorem 3.1.11. Let $f$ be a map of symmetric spectra such that $\pi_{*} f$ is an isomorphism of homotopy groups. Then $f$ is a stable equivalence.

Proof. To ease notation, let $R X=\operatorname{Hom}_{S}\left(F_{1} S^{1}, X\right)$, so that $R^{n} X=\operatorname{Hom}_{S}\left(F_{n} S^{n}, X\right)$ for $n \geq 0$ and $\operatorname{Ev}_{k} R^{n} X=\operatorname{Map}_{\mathcal{S}_{*}}\left(S^{n}, X_{n+k}\right)$. In particular $R^{0} X=X$ and there is a natural map $\lambda^{*}: X \rightarrow R X$ induced by the map $\lambda: F_{1} S^{1} \rightarrow F_{0} S^{0}$ discussed in Example 3.1.10 and Remark 2.2.12. The maps $R^{n}\left(\lambda^{*}\right): R^{n} X \rightarrow R^{n+1} X$ give a directed system. Let

$$
R^{\infty} X=\operatorname{colim}_{n \geq 0} R^{n} X=\operatorname{colim}_{n \geq 0} \operatorname{Hom}_{S}\left(F_{n} S^{n}, X\right)
$$

and let $r_{X}: X \rightarrow R^{\infty} X$ be the natural map from $X$ to the colimit.

Let $E$ be an injective $\Omega$-spectrum. Since $E$ is an $\Omega$-spectrum, the map

$$
R^{n}\left(\lambda^{*}\right): R^{n} E \rightarrow R^{n+1} E
$$

is a level equivalence for each $n$ and the map $r_{E}: E \rightarrow R^{\infty} E$ is a level equivalence. Since $E$ is injective, the proof of part one of Lemma 3.1.6 applied to $r_{E}$ shows that there is a map $g: R^{\infty} E \rightarrow E$ such that the composition $g r_{E}$ is simplicially homotopic to the identity map on $E$, though the other composite $r_{E} g$ may not be simplicially homotopic to the identity map on $R^{\infty} E$. There is a natural transformation $E^{0} X \rightarrow E^{0}\left(R^{\infty} X\right)$ sending the map $f: X \rightarrow E$ to the map $g R^{\infty} f$; there is a natural transformation $E^{0}\left(R^{\infty} X\right) \rightarrow E^{0} X$ induced by composition with the map $r_{X}: X \rightarrow R^{\infty} X$. Since $g r_{E}$ is simplicially homotopic to the identity map on $E$, the composition of the natural transformations is the identity natural transformation of the functor $E^{0}$. In the diagram

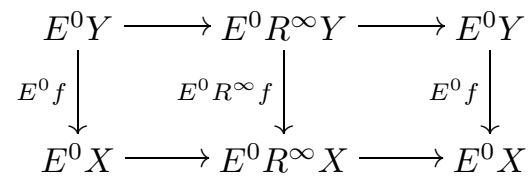

the composition of the horizontal maps is the identity, showing that $E^{0} f$ is a retract of $E^{0} R^{\infty} f$.

Let $X$ be a symmetric spectrum that is a level Kan complex, i.e., each $X_{n}$ is a Kan complex. Since the functor $\pi_{k}$ commutes with filtered colimits, the homotopy group $\pi_{k} \operatorname{Ev}_{n} R^{\infty} X$ of the pointed simplicial set $\operatorname{Ev}_{n} R^{\infty} X$ is naturally isomorphic to the homotopy group $\pi_{k-n} X$ of the symmetric spectrum $X$. A warning: even though the groups $\pi_{k} \operatorname{Ev}_{n} R^{\infty} X$ and $\pi_{k+1} \operatorname{Ev}_{n+1} R^{\infty} X$ are abstractly isomorphic, the structure map of the symmetric spectrum $R^{\infty} X$ need not induce an isomorphism between them. In particular, despite its similarity to the standard construction of the $\Omega$-spectrum associated to a (non-symmetric) spectrum, $R^{\infty} X$ need not be an $\Omega$-spectrum and $r_{X}: X \rightarrow R^{\infty} X$ need not induce an isomorphism of homotopy groups.

Now, let $f$ be a map of symmetric spectra such that $\pi_{*} f$ is an isomorphism. Assume as well that $X$ and $Y$ are level Kan complexes. Then $R^{\infty} f$ is a level equivalence. By Corollary 3.1.8, $E^{0} R^{\infty} f$ is an isomorphism for every injective $\Omega$ spectrum $E$. Thus $E^{0} f$, which is a retract of $E^{0} R^{\infty} f$, is an isomorphism for every injective $\Omega$-spectrum $E$, and so $f$ is a stable equivalence. 
To finish the proof, let $f: X \rightarrow Y$ be a map of arbitrary symmetric spectra such that $\pi_{*} f$ is an isomorphism. For every simplicial set $X$ there is a natural weak equivalence $X \rightarrow K X$ where $K X$ is a Kan complex. There are several such functors: $K$ can be Kan's $\mathrm{Ex}^{\infty}$ functor; $K$ can be the total singular complex of the geometric realization; or $K$ can be constructed using a simplicial small object argument. In each case, $K$ is an $\mathcal{S}_{*}$-functor and $X \rightarrow K X$ is an $\mathcal{S}_{*}$-natural transformation. By prolongation, for every symmetric spectrum $X$ there is a natural level equivalence $X \rightarrow K X$ where $K X$ is a level Kan complex. In the commutative diagram

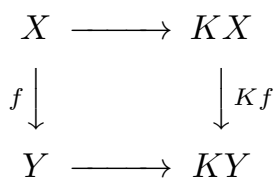

the horizontal maps are stable equivalences by 3.1.8; the map $K f$ is a stable equivalence by the preceding paragraph; hence $f$ is a stable equivalence.

As a corollary some of the standard results about spectra translate into results about symmetric spectra.

Definition 3.1.12. Let $(X, A)$ be a pair of symmetric spectra where $A$ is a subspectrum of $X$. The $k$ th relative homotopy group of the pair $(X, A)$ is the colimit

$$
\pi_{k}(X, A)=\operatorname{colim}_{n} \pi_{k+n}\left(X_{n}, A_{n} ; *\right)
$$

of the relative homotopy groups of the pointed pairs of simplicial sets $\left(X_{n}, A_{n} ; *\right)$.

Lemma 3.1.13 (Stable excision). Let $(X, A)$ be a pair of symmetric spectra with $A$ a subspectrum of $X$. The map of homotopy groups $\pi_{k}(X, A) \rightarrow \pi_{k}(X / A)$ is an isomorphism.

Proof. Consider the diagram:

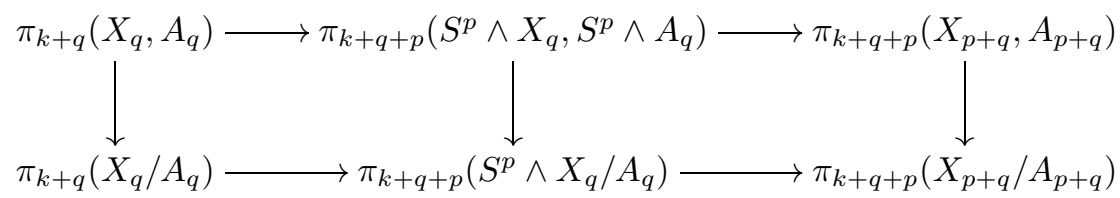

Let $(K, L)$ be a pair of pointed simplicial sets. By the homotopy excision theorem the map $\pi_{n}\left(S^{p} \wedge K, S^{p} \wedge L\right) \rightarrow \pi_{n}\left(S^{p} \wedge K / L\right)$ is an isomorphism when $n<2 p$. So the middle vertical arrow in the diagram is an isomorphism when $p>k+q$ and hence the map of colimits $\pi_{k}(X, A) \rightarrow \pi_{k}(X / A)$ is an isomorphism.

Theorem 3.1.14. (1) Let $f: X \rightarrow B$ be a map of symmetric spectra such that $f_{n}: X_{n} \rightarrow B_{n}$ is a Kan fibration for each $n \geq 0$ and let $F$ be the fiber over the basepoint. Then the map $X / F \rightarrow B$ is a stable equivalence.

(2) A map $f \in S p^{\Sigma}$ of symmetric spectra is a stable equivalence if and only if its suspension $f \wedge S^{1}$ is a stable equivalence.

(3) For symmetric spectra $X$ and $Y$ such that $Y$ is a level Kan complex, a map $X \rightarrow Y^{S^{1}}$ is a stable equivalence if and only its adjoint $X \wedge S^{1} \rightarrow Y$ is a stable equivalence.

Proof. By stable excision, the map $X / F \rightarrow B$ induces an isomorphism of homotopy groups and hence is a stable equivalence by Theorem 3.1.11. 
For part two, let $E$ be an injective $\Omega$-spectrum. By Lemma 3.1.2, the spectra $E^{S^{1}}, \operatorname{Hom}_{S}\left(F_{1} S^{0}, E\right)$ and $\operatorname{Hom}_{S}\left(F_{1} S^{1}, E\right)$ are injective $\Omega$-spectra. If $f$ is a stable equivalence of symmetric spectra, then the map

$$
\operatorname{Map}_{S p^{\Sigma}}\left(f, E^{S^{1}}\right)=\operatorname{Map}_{S p^{\Sigma}}\left(f \wedge S^{1}, E\right)
$$

is a weak equivalence of simplicial sets and so $f \wedge S^{1}$ is a stable equivalence. Conversely if $f \wedge S^{1}$ is a stable equivalence, then the map

$$
\operatorname{Map}_{S p^{\Sigma}}\left(f \wedge S^{1}, \operatorname{Hom}_{S}\left(F_{1} S^{0}, E\right)\right)=\operatorname{Map}_{S p^{\Sigma}}\left(f, \operatorname{Hom}_{S}\left(F_{1} S^{1}, E\right)\right)
$$

is a weak equivalence. The map $E \rightarrow \operatorname{Hom}_{S}\left(F_{1} S^{1}, E\right)$ is a level equivalence of injective spectra and thus a simplicial homotopy equivalence, by Lemma 3.1.6. So, for every symmetric spectrum $X$, the induced map

$$
\operatorname{Map}_{S p^{\Sigma}}(X, E) \rightarrow \operatorname{Map}_{S p^{\Sigma}}\left(X, \operatorname{Hom}_{S}\left(F_{1} S^{1}, E\right)\right)
$$

is a simplicial homotopy equivalence. Therefore $\operatorname{Map}_{S p^{\Sigma}}(f, E)$ is a weak equivalence, and so $f$ is a stable equivalence.

For part three, let $f: X \rightarrow Y^{S^{1}}$ be a map and let $f^{a}: X \wedge S^{1} \rightarrow Y$ be the adjoint of $f$. The diagram

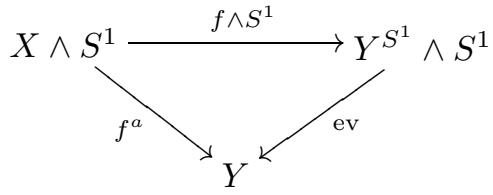

is commutative where the map ev is the evaluation map. By part one applied to the prolongation of the path fibration, the map ev is a stable equivalence; by part two, $f$ is a stable equivalence if and only if $f \wedge S^{1}$ is a stable equivalence. Therefore $f$ is a stable equivalence if and only if $f^{a}$ is a stable equivalence.

Once we have the stable model category of symmetric spectra, part three of this theorem tells us that it really is stable; i.e., that the suspension functor $-\wedge S^{1}$ is an equivalence of model categories.

3.2. Model categories. In this section we recall the definition and the basic properties of model categories; see [DS95], [Hov98a], or [DHK] for a more detailed introduction.

Definition 3.2.1. An ordered pair $(f, g)$ of maps in the category $\mathcal{C}$ has the lifting property if every commutative square

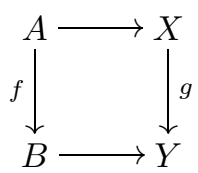

in $\mathcal{C}$ extends to a commutative diagram

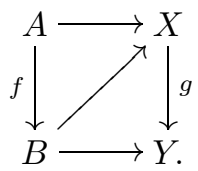

We also say that $f$ has the left lifting property with respect to $g$ and that $g$ has the right lifting property with respect to $f$. More generally, if $I$ and $J$ are classes 
of maps in $\mathcal{C}$, the pair $(I, J)$ has the lifting property if every pair $(f, g)$ with $f \in I$ and $g \in J$ has the lifting property. We also say that $I$ has the left lifting property with respect to $J$ and that $J$ has the right lifting property with respect to $I$.

It would be more accurate to say that the pair $(f, g)$ has the lifting-extension property but we prefer the shorter term.

Definition 3.2.2. Let $f$ and $g$ be maps in a category $\mathcal{C}$. The map $f$ is a retract of $g$ if it is a retract in the category of arrows, i.e., if there is a commutative diagram

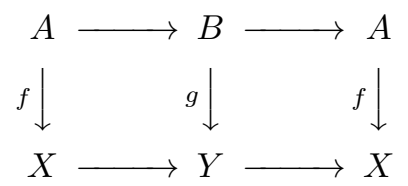

such that the horizontal compositions are the identity maps. A class of maps is closed under retracts if whenever $f$ is a retract of $g$ and $g$ is in the class, then $f$ is in the class.

Definition 3.2.3. A model category is a category $\mathcal{M}$ with three distinguished classes of maps - the class of weak equivalences, the class of cofibrations, and the class of fibrations - that satisfy the model category axioms below. We call a map that is both a cofibration and a weak equivalence a trivial cofibration, and we call a map that is both a fibration and a weak equivalence a trivial fibration.

M1 Limit axiom. The category $\mathcal{M}$ is bicomplete (closed under arbitrary small limits and colimits).

M2 Two-out-of-three axiom. Let $f$ and $g$ be maps in $\mathcal{M}$ such that $g f$ is defined. If two of $f, g$ and $g f$ are weak equivalences, then the third is a weak equivalence.

M3 Retract axiom. The class of weak equivalences, the class of cofibrations, and the class of fibrations are closed under retracts.

M4 Lifting axiom. A cofibration has the left lifting property with respect to every trivial fibration. A fibration has the right lifting property with respect to every trivial cofibration.

M5 Factorization axiom. Every map $f \in \mathcal{M}$ has a factorization $f=p i$ where $i$ is a cofibration and $p$ is a trivial fibration and a factorization $f=q j$ where $j$ is a trivial cofibration and $q$ is a fibration.

Three classes of maps that satisfy axioms M2, M3, M4 and M5 are a model structure on the category. One should keep in mind that a category can have more than one model structure; there can even be distinct model structures with the same class of weak equivalences.

A bicomplete category has an initial object $\emptyset$ and a terminal object $*$. In a model category, an object $X$ is cofibrant if the unique map $\emptyset \rightarrow X$ is a cofibration and an object $X$ is fibrant if the unique map $X \rightarrow *$ is a fibration. A model category is pointed if the unique map $\emptyset \rightarrow *$ is an isomorphism.

The following proposition is standard; see [Hov98a, Lemma 1.1.9].

Proposition 3.2.4 (The Retract Argument). Let $\mathcal{C}$ be a category and let $f=p i$ be a factorization in $\mathrm{C}$.

(1) If $p$ has the right lifting property with respect to $f$, then $f$ is a retract of $i$.

(2) If $i$ has the left lifting property with respect to $f$, then $f$ is a retract of $p$.

The following proposition is a converse to the lifting axiom. 
Proposition 3.2.5 (Closure property). In a model category:

(1) The cofibrations are the maps having the left lifting property with respect to every trivial fibration.

(2) The trivial cofibrations are the the maps having the left lifting property with respect to every fibration.

(3) The fibrations are the maps having the right lifting property with respect to every trivial cofibration.

(4) The trivial fibrations are the maps having the right lifting property with respect to every cofibration.

Proof. Use the factorization axiom and the retract argument.

In particular, any two of the three classes of maps in a model category determine the third. For example, a weak equivalence is a map that factors as a trivial cofibration composed with a trivial fibration.

Example 3.2.6. We recall the standard model structure on the category of simplicial sets [Qui67, II.3]. A weak equivalence is a map whose geometric realization is a homotopy equivalence of $\mathrm{CW}$-complexes. The cofibrations are the monomorphisms and every simplicial set is cofibrant. Recall, the standard $n$-simplex is $\Delta[n]=\Delta(-, \bar{n})$. The boundary of $\Delta[n]$ is the subfunctor $\partial \Delta[n] \subseteq \Delta[n]$ of nonsurjective maps. For $0 \leq i \leq n$, the $i$ th horn of $\Delta[n]$ is the subfunctor $\Lambda^{i}[n] \subseteq \partial \Delta[n]$ of maps for which $i$ is not in the image. Geometrically, $\Lambda^{i}[n]$ is obtained from the boundary of $\Delta[n]$ by removing the $i$ th face. The fibrations are the Kan fibrations, the maps that have the right lifting property with respect to the maps $\Lambda^{i}[n] \rightarrow \Delta[n]$ for $n>0$ and $0 \leq i \leq n$; the fibrant simplicial sets are the Kan complexes, the simplicial sets that satisfy the Kan extension condition. A map is a trivial fibration (a fibration and a weak equivalence) if and only if it has the right lifting property with respect to the maps $\partial \Delta[n] \rightarrow \Delta[n]$. It follows that the pointed weak equivalences, the pointed monomorphisms, and the pointed (Kan) fibrations are a model structure on the category of pointed simplicial sets.

When constructing a model category, the factorization axiom can be the hardest to verify. After some preliminary definitions, Lemma 3.2.11 constructs functorial factorizations in the category of symmetric spectra.

Definition 3.2.7. Let $I$ be a class of maps in a category $\mathcal{C}$.

(1) A map is I-injective if it has the right lifting property with respect to every map in $I$. The class of $I$-injective maps is denoted $I$-inj.

(2) A map is I-projective if it has the left lifting property with respect to every map in $I$. The class of $I$-projective maps is denoted $I$-proj.

(3) A map is an I-cofibration if it has the left lifting property with respect to every $I$-injective map. The class of $I$-cofibrations is the class $(I$-inj)-proj and is denoted $I$-cof.

(4) A map is an I-fibration if it has the right lifting property with respect to every $I$-projective map. The class of $I$-fibrations is the class ( $I$-proj)-inj and is denoted $I$-fib.

Injective and projective are dual notions; an $I$-injective map in $\mathcal{C}$ is an $I$ projective map in $\mathcal{C}^{\text {op }}$; an $I$-fibration in $\mathcal{C}$ is an $I$-cofibration in $\mathcal{C}^{\text {op }}$. The class $I$-inj and the class $I$-proj are analogous to the orthogonal complement of a set of 
vectors. This analogy helps explain the following proposition, whose proof we leave to the reader.

Proposition 3.2.8. Let $I$ and $J$ be classes of maps in a category $\mathcal{C}$.

(1) If $I \subseteq J$, then $J$-inj $\subseteq I$-inj and $J$-proj $\subseteq I$-proj.

(2) Repeating the operations: $I \subseteq I$-cof, $I \subseteq I$-fib, $I$-proj $=(I$-proj $)$-cof $=$ $(I$-fib $)$-proj, and $I$-inj $=(I$-inj $)$-fib $=(I$-cof $)$-inj.

(3) The following conditions are equivalent:

- The pair $(I, J)$ has the lifting property.

- $J \subseteq I$-inj.

- $I \subseteq J$-proj.

- The pair (I-cof, $J)$ has the lifting property.

- The pair $(I, J$-fib) has the lifting property.

(4) The classes I-inj and I-proj are subcategories of $\mathcal{C}$ and are closed under retracts.

(5) The class I-inj is closed under base change. That is, if

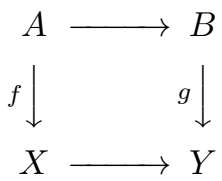

is a pullback square and $g$ is an I-injective map, then $f$ is an I-injective map.

(6) The class I-proj is closed under cobase change. That is, if

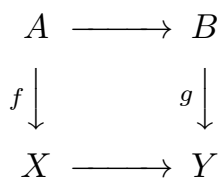

is a pushout square and $f$ is an I-projective map, then $g$ is an I-projective map.

Corollary 3.2.9. Let I be a class of maps in a category $\mathcal{C}$. The class $I$-cof is a subcategory of $\mathrm{C}$ that is closed under retracts and cobase change. The class $I$-fib is a subcategory of $\mathcal{C}$ that is closed under retracts and base change.

Another useful elementary lemma about the lifting property is the following.

Lemma 3.2.10. Let $L: \mathcal{C} \rightarrow \mathcal{D}$ be a functor that is left adjoint to the functor $R: \mathcal{D} \rightarrow \mathcal{C}$. If $I$ is a class of maps in $\mathcal{C}$ and $J$ is a class of maps in $\mathcal{D}$, the pair $(I, R J)$ has the lifting property if and only if the pair $(L I, J)$ has the lifting property.

The next lemma is used repeatedly to construct factorizations.

Lemma 3.2.11 (Factorization Lemma). Let I be a set of maps in the category $S p^{\Sigma}$. There is a functorial factorization of every map of symmetric spectra as an I-cofibration followed by an I-injective map.

The factorization lemma is proved using the transfinite small object argument. We begin by showing that every symmetric spectrum is suitably small.

Recall that an ordinal is, by recursive definition, the well-ordered set of all smaller ordinals. In particular, we can regard an ordinal as a category. A cardinal is an ordinal of larger cardinality than all smaller ordinals. 
Definition 3.2.12. Let $\gamma$ be an infinite cardinal. An ordinal $\alpha$ is $\gamma$-filtered if every set $A$ consisting of ordinals less than $\alpha$ such that $\sup A=\alpha$ has cardinality greater than $\gamma$.

Every $\gamma$-filtered ordinal is a limit ordinal. In fact, since $\gamma$ is infinite, every $\gamma$ filtered ordinal is a limit ordinal $\alpha$ for which there is no countable set $A$ of ordinals less than $\alpha$ such that $\sup A=\alpha$. The smallest $\gamma$-filtered ordinal is the first ordinal of cardinality greater than $\gamma$. For example, $\omega_{1}$ is the smallest $\aleph_{0}$-filtered ordinal. If $\gamma<\bar{\gamma}$ and $\alpha$ is $\bar{\gamma}$-filtered, then $\alpha$ is $\gamma$-filtered.

Define the cardinality of a spectrum $X$ to be the cardinality of its underlying set $\amalg_{n} \amalg_{k}\left(\operatorname{Ev}_{n} X\right)_{k}$. Then the cardinality of $X$ is always infinite, which is convenient for the following lemma.

Proposition 3.2.13. Let $X$ be a simplicial spectrum of cardinality $\gamma$. Let $\alpha$ be a $\gamma$-filtered ordinal and let $D: \alpha \rightarrow S p^{\Sigma}$ be an $\alpha$-indexed diagram of symmetric spectra. Then the natural map

$$
\operatorname{colim}_{\alpha} S p^{\Sigma}(X, D) \rightarrow S p^{\Sigma}\left(X, \operatorname{colim}_{\alpha} D\right)
$$

is an isomorphism.

Proof. Every symmetric spectrum has a presentation as a coequalizer

$$
S \otimes S \otimes X \longrightarrow S \otimes X \longrightarrow X
$$

of free symmetric spectra in the category $S p^{\Sigma}$. The symmetric spectra $X$ and $S \otimes X$ have the same cardinality. So the proposition follows once it is proved for free symmetric spectra. There is a natural isomorphism $S^{\Sigma}(S \otimes X, Y)=$ $\prod_{p} \mathcal{S}_{*}^{\Sigma_{p}}\left(X_{p}, Y_{p}\right)$. The functors $\mathcal{S}_{*}^{\Sigma_{p}}\left(X_{n},-\right)$ have the property claimed for $S p^{\Sigma}(X,-)$.

This fact is the heart of the proposition. To begin the proof of it, suppose we have a map $f: X_{n} \rightarrow \operatorname{colim}_{\alpha} D$, where $D$ is an $\alpha$-indexed diagram of $\Sigma_{p^{-}}$simplicial sets. For each simplex $x$ of $X_{n}$, we can choose an ordinal $\beta_{x}<\alpha$ and a simplex $y_{x} \in D_{\beta_{x}}$ such that $f(x)$ is the image of $y_{x}$. Because $\alpha$ is $\gamma$-filtered, we can then find one ordinal $\beta<\alpha$ and a map $g: X \rightarrow D_{\beta}$ factoring $f$. The map $g$ may not be simplicial or equivariant, but, again using the fact that $\alpha$ is $\gamma$-filtered, we can go out far enough in the colimit so that $g$ will be both simplicial and equivariant. We leave the details to the reader.

Since $\gamma$ is infinite, for every countable set $A$ of ordinals that are strictly less than $\alpha$, the ordinal $\sup A$ is strictly less than $\alpha$. Therefore, the countable product of functors $\mathcal{S}_{*}^{\Sigma}(X,-)$ has the property claimed for $S p^{\Sigma}(X,-)$ and the proposition is proved.

Proof of Lemma 3.2.11. We begin by constructing a functorial factorization

$$
X \stackrel{I g}{\longrightarrow} E g \stackrel{P g}{\longrightarrow} Y
$$

of $g: X \rightarrow Y$ such that $I g$ is an $I$-cofibration and $g=P g \circ I g$. For a map $f: b_{f} \rightarrow c_{f}$ in $I$, let $D f$ be the set of commutative squares

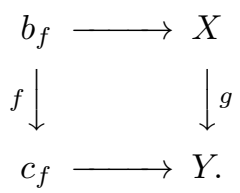


Let

$$
B=\amalg_{f \in I} \amalg_{D f} b_{f}, C=\amalg_{f \in I} \amalg_{D f} c_{f} \text {, and } F=\amalg_{f \in I} \amalg_{D f} f: B \rightarrow C .
$$

By the definition of $D f$, there is a commutative square

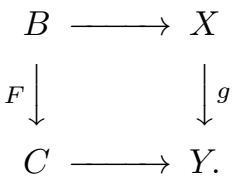

Let $E g$ be the pushout $X \amalg_{B} C$, let $I g$ be the map $X \rightarrow E g=X \amalg_{B} C$, and let $P g: E g \rightarrow Y$ be the natural map on the pushout. By construction, the map $I g: X \rightarrow X \amalg_{B} C$ is an $I$-cofibration and $g=P g \circ I g$. However, the map $P g$ need not be an $I$-injective map.

Use transfinite induction to define functorial factorizations of $g$

$$
X \stackrel{I^{\alpha} g}{\longrightarrow} E^{\alpha} g \stackrel{P^{\alpha} g}{\longrightarrow} Y
$$

for every ordinal $\alpha$. The induction starts at 0 with $E^{0} g=X, I^{0} g=i d_{X}$, and $P^{0} g=$ $g$. For a successor ordinal $\alpha+1, E^{\alpha+1} g=E\left(P^{\alpha} g\right), I^{\alpha+1} g=I\left(P^{\alpha} g\right) \circ I^{\alpha} g$, and $P^{\alpha+1} g=P\left(P^{\alpha} g\right)$. For $\beta$ a limit ordinal, $E^{\beta} g=\operatorname{colim}_{\alpha<\beta} E^{\alpha} g, I^{\beta} g=\operatorname{colim}_{\alpha<\beta} I^{\alpha} g$ and $P^{\beta} g=\operatorname{colim}_{\alpha<\beta} P^{\alpha} g$.

The map $I^{\alpha} g: X \rightarrow E^{\alpha} g$ is an $I$-cofibration for each $\alpha$; the required lift is constructed by transfinite induction. The proof of the lemma is completed by finding an ordinal $\beta$ for which $P_{\beta} g$ is an $I$-injective map. Let

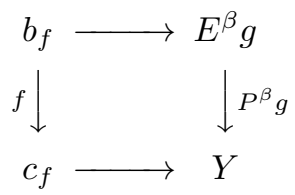

be a commutative square with $f \in I$. If the map $b_{f} \rightarrow E^{\beta} g$ factors as $b_{f} \rightarrow E^{\alpha} g \rightarrow$ $E^{\beta} g$ for $\alpha<\beta$, then by construction there is a lift $c_{f} \rightarrow E^{\alpha+1} g$ and, since $\alpha+1 \leq \beta$, a lift $c_{f} \rightarrow E^{\beta} g$. Let $\gamma_{f}$ be the cardinality of $b_{f}$ and let $\gamma=\sup _{I} \gamma_{f}$. Let $\beta$ be a $\gamma$-filtered ordinal. Then $S p^{\Sigma}\left(b_{f}, P^{\beta} g\right)=\operatorname{colim}_{\alpha<\beta} S p^{\Sigma}\left(b_{f}, P^{\alpha} g\right)$ for every $f \in I$ and hence $P^{\beta} g$ is an $I$-injective map.

3.3. Level structure. Prolongation of the model structure on $\mathcal{S}_{*}$ (see 3.2.6) gives the level structure on the category of symmetric spectra. It is not a model structure but it is a basic tool in the construction of the stable model structure. Its use is already implicit in Sections 1.3 and 3.1.

Definition 3.3.1. Let $f: X \rightarrow Y$ be a map of symmetric spectra.

(1) The map $f$ is a level equivalence if each map $f_{n}: X_{n} \rightarrow Y_{n}$ is a weak equivalence of simplicial sets.

(2) The map $f$ is a level (trivial) cofibration if each map $f_{n}: X_{n} \rightarrow Y_{n}$ is a (trivial) cofibration of simplicial sets.

(3) The map $f$ is a level (trivial) fibration if each map $f_{n}: X_{n} \rightarrow Y_{n}$ is a (trivial) fibration of simplicial sets.

The level cofibrations are the monomorphisms of symmetric spectra. Next, we characterize the level fibrations and trivial fibrations. 
Definition 3.3.2. (1) Let $I_{\Lambda}$ denote the set of maps $\Lambda^{k}[r]_{+} \rightarrow \Delta[r]_{+}$for $r>0$ and $0 \leq k \leq r$. Let $F I_{\Lambda}=\bigcup_{n \geq 0} F_{n}\left(I_{\Lambda}\right)$.

(2) Let $I_{\partial}$ denote the set of maps $\partial \Delta[r]_{+} \rightarrow \Delta[r]_{+}$for $r \geq 0$. Let $F I_{\partial}=$ $\bigcup_{n \geq 0} F_{n}\left(I_{\partial}\right)$.

Proposition 3.3.3. The level fibrations are the $F I_{\Lambda}$-injective maps. The level trivial fibrations are the $F I_{\partial}$-injective maps.

Proof. A map $g$ is a level (trivial) fibration if and only if $\operatorname{Ev}_{n} g=g_{n}$ is a (trivial) Kan fibration for each $n \geq 0$. But $\operatorname{Ev}_{n} g$ is a (trivial) Kan fibration if and only if it has the right lifting property with respect to the class $\left(I_{\partial}\right) I_{\Lambda}$. Then by adjunction, $g$ is a level (trivial) fibration if and only if $g$ has the right lifting property with respect to the class $\left(F I_{\partial}\right) F I_{\Lambda}$.

The level structure is not a model structure; it satisfies the two-out-of-three axiom, the retract axiom, and the factorization axiom but not the lifting axiom. A model structure is determined by any two of its three classes and so the level structure is overdetermined. In Section 5.1 we prove there are two "level" model structures with the level equivalences as the weak equivalences: one that is generated by the level equivalences and the level cofibrations and one that is generated by the level equivalences and the level fibrations. In any case, the level homotopy category obtained by inverting the level equivalences is not the stable homotopy category of spectra.

The pushout smash product (Definition 1.3.3) has an adjoint construction.

Definition 3.3.4. Let $f: U \rightarrow V$ and $g: X \rightarrow Y$ be maps of pointed simplicial sets. The map

$$
\operatorname{Map}_{\square}(f, g): \operatorname{Map}(V, X) \rightarrow \operatorname{Map}(U, X) \times_{\operatorname{Map}(U, Y)} \operatorname{Map}(V, Y)
$$

is the map to the fiber product induced by the commutative square

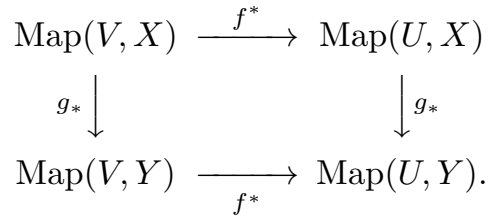

Let $f$ be a map of pointed simplicial sets and let $g$ be a map of symmetric spectra. Then $\operatorname{Hom}_{\square}(f, g)$ is the map of symmetric spectra that is defined by prolongation, $\operatorname{Ev}_{n} \operatorname{Hom}_{\square}(f, g)=\operatorname{Map}_{\square}\left(f, g_{n}\right)$.

Proposition 3.3.5. (1) If $f \in \mathcal{S}_{*}$ is a monomorphism and $g \in \mathcal{S}_{*}$ is a Kan fibration, then $\operatorname{Map}_{\square}(f, g)$ is a Kan fibration. If, in addition, either $f$ or $g$ is a weak equivalence, then $\operatorname{Map}_{\square}(f, g)$ is a weak equivalence.

(2) If $f \in \mathcal{S}_{*}$ is a monomorphism and $g \in S p^{\Sigma}$ is a level fibration, then $\operatorname{Hom}_{\square}(f, g)$ is a level fibration. If, in addition, either $f$ is a weak equivalence or $g$ is a level equivalence, then $\operatorname{Hom}_{\square}(f, g)$ is a level equivalence.

Proof. Part one is a standard property of simplicial sets, proved in [Qui67, II.3]. Part two follows from part one by prolongation.

Definition 3.3.6. Let $f: U \rightarrow V$ and $g: X \rightarrow Y$ be maps in a category $\mathcal{C}$. Then $\mathcal{C}_{\square}(f, g)$ is the natural map of sets to the fiber product

$$
\mathcal{C}(V, X) \rightarrow \mathcal{C}(U, X) \times e_{(U, Y)} \mathcal{e}(V, Y)
$$


coming from the commutative square

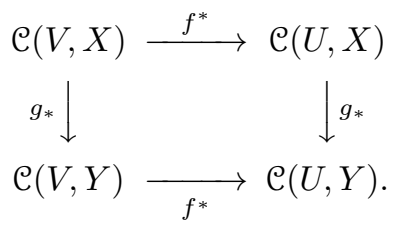

A pair $(f, g)$ has the lifting property if and only if $\mathcal{C}_{\square}(f, g)$ is surjective.

Definition 3.3.7. Let $f: U \rightarrow V$ and $g: X \rightarrow Y$ be maps of symmetric spectra. Then $\operatorname{Map}_{\square}(f, g)$ is the natural map to the fiber product

$$
S p_{\square}^{\Sigma}\left(f \wedge \Delta[-]_{+}, g\right): \operatorname{Map}(V, X) \rightarrow \operatorname{Map}(U, X) \times_{\operatorname{Map}(U, Y)} \operatorname{Map}(V, Y) .
$$

Proposition 3.3.8. Let $f$ and $h$ be maps of symmetric spectra and let $g$ be a map of pointed simplicial sets. There are natural isomorphisms

$$
S p_{\square}^{\Sigma}(f \square g, h) \cong\left(\mathcal{S}_{*}\right)_{\square}\left(g, \operatorname{Map}_{\square}(f, h)\right) \cong S p_{\square}^{\Sigma}\left(f, \operatorname{Hom}_{\square}(g, h)\right) .
$$

In fact this proposition holds in any simplicial model category.

Proof. Let $f: U \rightarrow V$ and $h: X \rightarrow Y$ be maps in $S p^{\Sigma}$ and let $g: K \rightarrow L$ be a map in $\mathcal{S}_{*}$. Using adjunction and the defining property of pushouts and of pullbacks, each of the three maps in the proposition is naturally isomorphic to the map from $S p^{\Sigma}(V \wedge L, X)$ to the limit of the diagram:

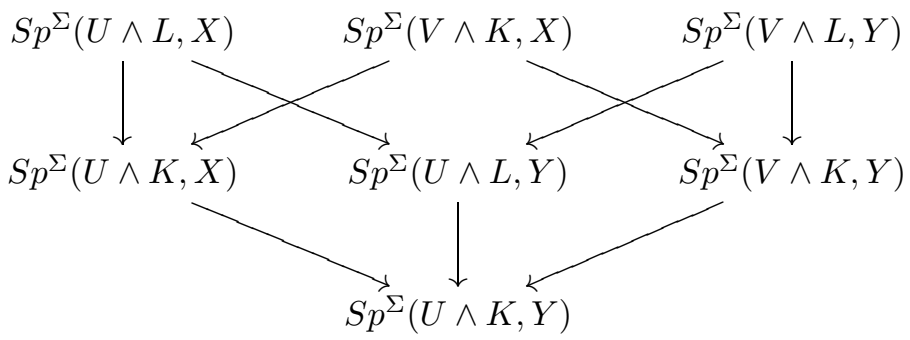

Corollary 3.3.9. Let $f$ and $h$ be maps of symmetric spectra and let $g$ be a map of pointed simplicial sets. The following are equivalent:

- $(f \square g, h)$ has the lifting property.

- $\left(g, \operatorname{Map}_{\square}(f, h)\right)$ has the lifting property.

- $\left(f, \operatorname{Hom}_{\square}(g, h)\right)$ has the lifting property.

3.4. Stable model category. In this section we define the stable cofibrations and the stable fibrations of symmetric spectra. The main result is that the class of stable equivalences, the class of stable cofibrations, and the class of stable fibrations are a model structure on $S p^{\Sigma}$.

Recall that $f$ is a level trivial fibration if $f_{n}$ is a trivial Kan fibration for each $n \geq 0$.

Definition 3.4.1. A map of symmetric spectra is a stable cofibration if it has the left lifting property with respect to every level trivial fibration. A map of symmetric spectra is a stable trivial cofibration if it is a stable cofibration and a stable equivalence. A symmetric spectrum $X$ is stably cofibrant if $* \rightarrow X$ is a stable cofibration. 
The basic properties of the class of stable cofibrations are next.

Proposition 3.4.2. (1) The class of stable cofibrations is the class $F I_{\partial}$-cof.

(2) The class of stable cofibrations is a subcategory that is closed under retracts and closed under cobase change.

(3) If $f$ is a cofibration of pointed simplicial sets and $n \geq 0$, then $F_{n} f$ is a stable cofibration. In particular, $F_{n} K$ is stably cofibrant for $K \in \mathcal{S}_{*}$.

(4) If $f \in S p^{\Sigma}$ is a stable cofibration and $g \in \mathcal{S}_{*}$ is a cofibration, then the pushout smash product $f \square g$ is a stable cofibration.

(5) If $f \in S p^{\Sigma}$ is a stable cofibration and $h \in S p^{\Sigma}$ is a level fibration, then $\operatorname{Map}_{\square}(f, h)$ is a Kan fibration.

Proof. The stable cofibrations are the maps having the left lifting property with respect to the level trivial fibrations, which by Proposition 3.3.3 are the $F I_{\partial}$-injective maps. So the stable cofibrations are the $F I_{\partial}$-cofibrations.

Every class $I$-cof has the properties stated in part two.

Suppose $g \in S p^{\Sigma}$ is a level trivial fibration, and $f \in \mathcal{S}_{*}$ is a cofibration. Then $f$ has the left lifting property with respect to the trivial Kan fibration $\operatorname{Ev}_{n} g$. By adjunction, $F_{n} f$ has the left lifting property with respect to $g$. Hence $F_{n} f$ is a stable cofibration. In particular, for every pointed simplicial set $K$, the map $* \rightarrow F_{n} K$ is a stable cofibration, and so $F_{n} K$ is stably cofibrant.

Now suppose $f \in S p^{\Sigma}$ is a stable cofibration and $g \in \mathcal{S}_{*}$ is a cofibration. Then, given a level trivial fibration $h \in S p^{\Sigma}$, the map $\operatorname{Hom}_{\square}(g, h)$ is a level trivial fibration by Proposition 3.3.5. Therefore the pair $\left(f, \operatorname{Hom}_{\square}(g, h)\right)$ has the lifting property. Then by Corollary 3.3.9, the pair $(f \square g, h$ ) has the lifting property, and so $f \square g$ is a stable cofibration.

Finally, suppose $f \in S p^{\Sigma}$ is a stable cofibration and $h \in S p^{\Sigma}$ is a level fibration. Given a trivial cofibration $g \in \mathcal{S}_{*}$, $\operatorname{Hom}_{\square}(g, h)$ is a level trivial fibration by Proposition 3.3.5. Therefore, the pair $\left(f, \operatorname{Hom}_{\square}(g, h)\right)$ has the lifting property. Then, by Corollary 3.3 .9 , the pair $\left(g, \operatorname{Map}_{\square}(f, h)\right)$ has the lifting property. Therefore $\operatorname{Map}_{\square}(f, h)$ is a Kan fibration.

The next definition is natural in view of the closure properties in a model category; see Proposition 3.2.5.

Definition 3.4.3. A map of symmetric spectra is a stable fibration if it has the right lifting property with respect to every map that is a stable trivial cofibration. A map of symmetric spectra is a stable trivial fibration if it is a stable fibration and a stable equivalence. A spectrum $X$ is stably fibrant if the map $X \rightarrow *$ is a stable fibration.

Theorem 3.4.4. The category of symmetric spectra with the class of stable equivalences, the class of stable cofibrations, and the class of stable fibrations is a model category.

Proof. The category $S p^{\Sigma}$ is bicomplete by Proposition 1.2.10. The two-out-ofthree axiom and the retract axiom are immediate consequences of the definitions. By definition, $(i, p)$ has the lifting property when $i$ is a stable trivial cofibration and $p$ is a stable fibration. The lifting axiom for $i$ a stable cofibration and $p$ a stable trivial fibration is verified in Corollary 3.4.7. The two parts of the factorization axiom are verified in Corollary 3.4.6 and Lemma 3.4.8. 
Lemma 3.4.5. A map is a stable trivial fibration if and only if it is a level trivial fibration.

Proof. Suppose $g$ is a level trivial fibration. By definition, every stable cofibration has the left lifting property with respect to $g$ and in particular every stable trivial cofibration has the left lifting property with respect to $g$. So $g$ is a stable fibration which is a level equivalence and hence a stable equivalence. So $g$ is a stable trivial fibration.

Conversely, suppose $g$ is a stable trivial fibration. Recall that at this point we do not know that $g$ has the right lifting property with respect to stable cofibrations. By Lemma 3.2.11, $g$ can be factored as $g=p i$ with $i$ an $F I_{\partial}$-cofibration and $p$ an $F I_{\partial}$-injective map. Since $p$ is a level equivalence, it is a stable equivalence. By the two-out-of-three property, $i$ is a stable equivalence. Therefore, $i$ is a stable trivial cofibration and has the left lifting property with respect to $g$. By the Retract Argument 3.2.4, $g$ is a retract of $p$ and so $g$ is a level trivial fibration.

Corollary 3.4.6. Every map $f$ of symmetric spectra has a factorization $f=p i$ as a stable cofibration $i$ followed by a stable trivial fibration $p$.

Proof. By the Factorization Lemma 3.2.11, every map $f$ in $S p^{\Sigma}$ can be factored as $f=p i$ with $i$ an $F I_{\partial}$-cofibration and $p$ an $F I_{\partial}$-injective map. Then $i$ is a stable cofibration and $p$ is a level trivial fibration, which, by Lemma 3.4.5, means that $p$ is a stable trivial fibration.

Corollary 3.4.7. A stable cofibration has the left lifting property with respect to every stable trivial fibration.

Proof. By Lemma 3.4.5 every stable trivial fibration is a level trivial fibration. By definition, stable cofibrations have the left lifting property with respect to every level trivial fibration.

The following lemma will finish the proof of Theorem 3.4.4.

Lemma 3.4.8. Every map $f$ of symmetric spectra has a factorization $f=p i$ as a stable trivial cofibration $i$ followed by a stable fibration $p$.

To prove the lemma we need a set of maps $J$ such that a $J$-cofibration is a stable trivial cofibration and a $J$-injective map is a stable fibration. Using the Factorization Lemma with the set $J$ will prove Lemma 3.4.8. The set $J$ is defined in 3.4.9 and Corollary 3.4.16 verifies its properties. This takes up the rest of the section.

The maps $\lambda \wedge F_{n} S^{0}$ used in the definition below appeared in the description of the function spectrum in Remark 2.2.12. They are stable equivalences (see Example 3.1.10) but are not stable cofibrations or even level cofibrations. We modify them to get the set $J$.

Definition 3.4.9. Let $\lambda: F_{1} S^{1} \rightarrow F_{0} S^{0}$ be the adjoint of the identity map $S^{1} \rightarrow$ $\operatorname{Ev}_{1} F_{0} S^{0}=S^{1}$ and let $\lambda_{n}$ be the map $\lambda \wedge F_{n} S^{0}: F_{n+1} S^{1} \rightarrow F_{n} S^{0}$, so that $\lambda_{0}=\lambda$. The mapping cylinder construction 3.1 .7 gives a factorization $\lambda_{n}=r_{n} c_{n}$ where $r_{n}: C \lambda_{n} \rightarrow F_{n} S^{0}$ is a simplicial homotopy equivalence and $c_{n}: F_{n+1} S^{1} \rightarrow C \lambda_{n}$ is a level cofibration. For $n \geq 0$, let $K_{n}=c_{n} \square I_{\partial}$, i.e., $K_{n}$ is the set of maps $c_{n} \square j$ for $j \in I_{\partial}$. Let $K=\bigcup_{n} K_{n}$ and, finally, let $J=F I_{\Lambda} \cup K$. 
Lemma 3.4.10. For each $n \geq 0$, the map $c_{n}: F_{n+1} S^{1} \rightarrow C \lambda_{n}$ is a stable trivial cofibration.

Proof. The map $\lambda_{n}$ is a stable equivalence (Example 3.1.10) and the simplicial homotopy equivalence $r_{n}$ is a stable equivalence. Using the factorization $\lambda_{n}=r_{n} c_{n}$ and the two-out-of-three property of stable equivalences, $c_{n}$ is a stable equivalence.

Next we show that $c_{n}$ is a stable cofibration. The mapping cylinder $C \lambda_{n}$ can also be defined as the corner in the pushout square

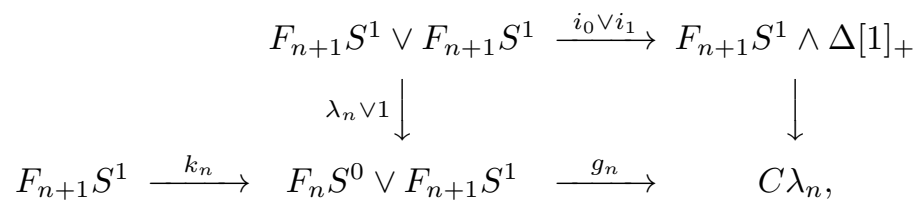

where $k_{n}$ is the inclusion on the second factor, $i_{0}$ and $i_{1}$ come from the two inclusions $\Delta[0] \rightarrow \Delta[1]$, and $g_{n}$ is the natural map to the pushout. Using the properties of stable cofibrations in Proposition 3.4.2, we find that the map $* \rightarrow F_{n} S^{0}$ is a stable cofibration and, by cobase change, that $k_{n}$ is a stable cofibration. Let $j$ be the cofibration $\partial \Delta[1]_{+} \rightarrow \Delta[1]_{+}$. Then $\left(* \rightarrow F_{n+1} S^{1}\right) \square j=i_{0} \vee i_{1}$ is a stable cofibration and, by cobase change, $g_{n}$ is a stable cofibration. Thus the composition $c_{n}=g_{n} k_{n}$ is a stable cofibration.

Next we characterize the $J$-injective maps.

Definition 3.4.11. A commutative square of simplicial sets

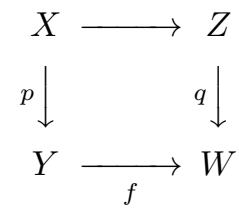

where $p$ and $q$ are fibrations is a homotopy pullback square if the following equivalent conditions hold:

- The induced map $X \rightarrow Y \times_{W} Z$ is a weak equivalence.

- For every 0 -simplex $v \in Y_{0}$, the map of fibers $p^{-1} v \rightarrow q^{-1} f v$ is a weak equivalence.

Lemma 3.4.12. A map of symmetric spectra $p: E \rightarrow B$ is $J$-injective if and only if $p$ is a level fibration and the diagram

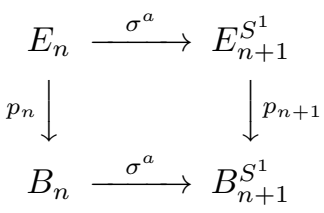

is a homotopy pullback square for each $n \geq 0$, where the horizontal maps are the adjoints of the structure maps.

Proof. Since $J=F I_{\Lambda} \cup K$ and $K=\bigcup_{n} K_{n}$, a map is $J$-injective if and only if it is $F I_{\Lambda^{-}}$-injective and $K_{n}$-injective for each $n \geq 0$. By Proposition 3.3.3, the $F I_{\Lambda^{-}}$ injective maps are the level fibrations. By definition, $p \in S p^{\Sigma}$ is a $K_{n}$-injective map if and only if $p$ has the right lifting property with respect to the class $c_{n} \square I_{\partial}$. Then, by Corollary 3.3.9, $p$ is $K_{n}$-injective if and only if $\operatorname{Map}_{\square}\left(c_{n}, p\right)$ has the right 
lifting property with respect to the class $I_{\partial}$. Hence, $p$ is $K_{n}$-injective if and only if $\operatorname{Map}_{\square}\left(c_{n}, p\right)$ is a trivial Kan fibration. If $p$ is a level fibration, $\operatorname{Map}_{\square}\left(c_{n}, p\right)$ is a Kan fibration by Proposition 3.4.2. So, a level fibration $p$ is $K_{n}$-injective if and only if $\operatorname{Map}_{\square}\left(c_{n}, p\right)$ is a weak equivalence. Taken together, $p$ is $J$-injective if and only if $p$ is a level fibration and $\operatorname{Map}_{\square}\left(c_{n}, p\right)$ is a weak equivalence for each $n \geq 0$.

For each $n \geq 0$, the map $r_{n}: C \lambda_{n} \rightarrow F_{n} S^{0}$ has a simplicial homotopy inverse $s_{n}: F_{n} S^{0} \rightarrow C \lambda_{n}$ for which $r_{n} s_{n}$ is the identity map on $F_{n} S^{0}$ (see 3.1.7). Then $\operatorname{Map}_{\square}\left(c_{n}, p\right)$ is simplicially homotopic to $\operatorname{Map}_{\square}\left(s_{n} \lambda_{n}, p\right)$. Since $F_{n} S^{0}$ is a simplicial deformation retract of $C \lambda_{n}, \lambda_{n}$ is a simplicial deformation retract of $s_{n} \lambda_{n}$ and $\operatorname{Map}_{\square}\left(\lambda_{n}, p\right)$ is a simplicial deformation retract of $\operatorname{Map}_{\square}\left(s_{n} \lambda_{n}, p\right)$. Therefore, $\operatorname{Map}_{\square}\left(c_{n}, p\right)$ is a weak equivalence if and only if $\operatorname{Map}_{\square}\left(\lambda_{n}, p\right)$ is a weak equivalence.

The map

$$
\operatorname{Map}_{\square}\left(\lambda_{n}, p\right): \operatorname{Map}\left(F_{n} S^{0}, E\right) \rightarrow \operatorname{Map}\left(F_{n} S^{0}, B\right) \times_{\operatorname{Map}\left(F_{n+1} S^{1}, B\right)} \operatorname{Map}\left(F_{n+1} S^{1}, E\right)
$$

is naturally isomorphic to the map

$$
E_{n} \rightarrow B_{n} \times{ }_{B_{n+1}^{S^{1}}} E_{n+1}^{S^{1}}
$$

induced by the diagram $(*)$. If $p$ is a level fibration, then by definition the diagram $(*)$ is a homotopy pullback square if and only if the map $\operatorname{Map}_{\square}\left(\lambda_{n}, p\right)$ is a weak equivalence.

Combining the conclusions of the three paragraphs completes the proof.

Corollary 3.4.13. The map $F \rightarrow *$ is $J$-injective if and only if $F$ is an $\Omega$ spectrum.

We also get the following corollary, which is not needed in the sequel. Its proof uses properness (see Section 5.5).

Corollary 3.4.14. A level fibration between two $\Omega$-spectra is $J$-injective.

Lemma 3.4.15. Let $p: X \rightarrow Y$ be a map of symmetric spectra. If $p$ is $J$-injective and $p$ is a stable equivalence, then $p$ is a level equivalence.

Proof. Suppose $p: X \rightarrow Y$ is a $J$-injective stable equivalence. In particular, $p$ is a level fibration. Let $F$ be the fiber over the basepoint. Since the class $J$-inj is closed under base change, the map $F \rightarrow *$ is $J$-injective and $F$ is an $\Omega$-spectrum. The map $p$ factors as $X \rightarrow X / F \rightarrow Y$. The map $X / F \rightarrow Y$ is a stable equivalence by Theorem 3.1.14. Since $p: X \rightarrow Y$ is a stable equivalence, $q: X \rightarrow X / F$ is a stable equivalence.

A Barratt-Puppe type sequence for symmetric spectra is constructed by prolongation to give the diagram

$$
X \rightarrow X \amalg_{F}\left(F \wedge \Delta[1]_{+}\right) \rightarrow F \wedge S^{1} \rightarrow X \wedge S^{1} \rightarrow\left(X \amalg_{F}\left(F \wedge \Delta[1]_{+}\right)\right) \wedge S^{1} .
$$

Let $E$ be an injective $\Omega$-spectrum. Since the map $X \amalg_{F}\left(F \wedge \Delta[1]_{+}\right) \rightarrow X / F$ is a level equivalence, after applying $E^{0}(-)$ to this sequence we can rewrite the terms involving the homotopy cofiber as $E^{0}(X / F)$. This gives an exact sequence

$$
E^{0} X \stackrel{E^{0} q}{\longleftarrow} E^{0}(X / F) \leftarrow E^{0}\left(F \wedge S^{1}\right) \leftarrow E^{0}\left(X \wedge S^{1}\right) \stackrel{E^{0}\left(q \wedge S^{1}\right)}{\longleftarrow} E^{0}\left(X / F \wedge S^{1}\right) .
$$

Since $q: X \rightarrow X / F$ is a stable equivalence, $E^{0} q$ is an isomorphism by definition, and $E^{0}\left(q \wedge S^{1}\right)$ is an isomorphism by part two of Theorem 3.1.14. Hence, $E^{0}\left(F \wedge S^{1}\right)=*$ for every injective $\Omega$-spectrum $E$, and so, by part two of Theorem $3.1 .14, E^{0} F=*$ for every injective $\Omega$-spectrum $E$. By Corollary 5.1.3, there is a level equivalence 
$F \rightarrow E$ where $E$ is an injective spectrum; since $F$ is an $\Omega$-spectrum, $E$ is an injective $\Omega$-spectrum. By Lemma 3.1.6, $E^{0} E=E^{0} F=*$. So $E$ is simplicially homotopic to $*$ and $F$ is level equivalent to $*$.

This does not finish the argument as the base of the fibration $X_{n} \rightarrow Y_{n}$ need not be connected. Since $p$ is $J$-injective,

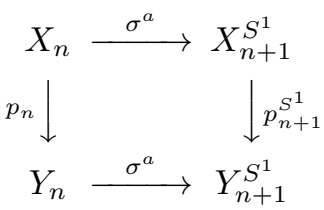

is a homotopy pullback square for each $n \geq 0$. The proof is completed by showing that $p_{n+1}^{S^{1}}$ is a trivial Kan fibration for every $n \geq 0$ which implies that $p_{n}$ is a trivial Kan fibration for every $n \geq 0$. For a pointed simplicial set $K$, let $c K$ denote the connected component of the basepoint. If $E \rightarrow B$ is a pointed Kan fibration, then $c E \rightarrow c B$ is a Kan fibration; if the fiber over the basepoint $* \in B$ is contractible, then $c E \rightarrow c B$ is a trivial Kan fibration. In particular, $c X_{n} \rightarrow c Y_{n}$ is a trivial Kan fibration and, therefore, $\left(c X_{n}\right)^{S^{1}} \rightarrow\left(c Y_{n}\right)^{S^{1}}$ is a trivial fibration. Since $K^{S^{1}}=(c K)^{S^{1}}$ for any pointed simplicial set $K, p_{n}^{S^{1}}: X_{n}^{S^{1}} \rightarrow Y_{n}^{S^{1}}$ is a trivial Kan fibration for every $n \geq 0$.

The next corollary finishes the proof of Lemma 3.4.8.

Corollary 3.4.16. The J-cofibrations are the stable trivial cofibrations and the $J$-injective maps are the stable fibrations.

Proof. Every level trivial fibration is $J$-injective since it satisfies the condition in Lemma 3.4.12. Thus, a $J$-cofibration has the left lifting property with respect to every level trivial fibration, and hence a $J$-cofibration is a stable cofibration. Let $E$ be an $\Omega$-spectrum. The maps $p: E \rightarrow *$ and $q=\operatorname{Hom}(j, E): E^{\Delta[1]} \rightarrow E \times E$, where $j: \partial \Delta[1]_{+} \rightarrow \Delta[1]_{+}$is the inclusion, are $J$-injective by Lemma 3.4.12. Let $E$ be an injective $\Omega$-spectrum and $f$ be a $J$-cofibration. Since $f$ has the left lifting property with respect to $p: E \rightarrow *, E^{0} f$ is surjective. Since $f$ has the left lifting property with respect to $q$ : $E^{\Delta[1]} \rightarrow E \times E, E^{0} f$ is injective. So $E^{0} f$ is an isomorphism and every $J$-cofibration is a stable trivial cofibration.

Conversely, let $f$ be a stable trivial cofibration. By the Factorization Lemma 3.2.11, $f$ factors as $f=p i$ where $i$ is a $J$-cofibration and $p$ is a $J$-injective map. We have just seen that $i$ is a stable equivalence. So, the $J$-injective map $p$ is a stable equivalence and by Lemma 3.4.15, $p$ is a level equivalence. Therefore the stable cofibration $f$ has the left lifting property with respect to the map $p$. By the Retract Argument 3.2.4, $f$ is a $J$-cofibration.

Let $F$ be the class of stable fibrations. Since $J$-cof is the class of stable trivial cofibrations, one has by the definition of stable fibrations that $F=(J$-cof $)$-inj. But $(J$-cof)-inj $=J$-inj by Proposition 3.2.8 (2). In other words, the stable fibrations are the $J$-injective maps.

In particular, Lemma 3.4.12 characterizes the stable fibrations. The stably fibrant objects are the $\Omega$-spectra by Corollary 3.4.13. Corollary 3.4.16 finishes the proof of Lemma 3.4.8 and the verification of the axioms for the stable model category of symmetric spectra. 


\section{Comparison with the Bousfield-Friedlander Category}

The goal of this section is to show that the stable homotopy theory of symmetric spectra and the stable homotopy theory of spectra are equivalent. We begin in Section 4.1 by recalling the general theory of Quillen equivalences of model categories. In Section 4.2 we provide a brief recap of the stable homotopy theory of (non-symmetric) spectra and we show that the forgetful functor $U$ from symmetric spectra to spectra is part of a Quillen equivalence. The left adjoint $V$ of $U$ plays very little role in this proof, beyond its existence, so we postpone its construction to Section 4.3.

4.1. Quillen equivalences. In this section, we briefly recall Quillen functors and Quillen equivalences between model categories.

Definition 4.1.1. Let $\mathcal{C}$ and $\mathcal{D}$ be model categories. Let $L: \mathcal{C} \rightarrow \mathcal{D}$ and $R: \mathcal{D} \rightarrow \mathcal{C}$ be functors such that $L$ is left adjoint to $R$. The adjoint pair of functors $L$ and $R$ is a Quillen adjoint pair if $L$ preserves cofibrations and $R$ preserves fibrations. We refer to the functors in such a pair as left and right Quillen functors. A Quillen adjoint pair is a Quillen equivalence if for every cofibrant object $X \in \mathcal{C}$ and every fibrant object $Y \in \mathcal{D}$, a map $L X \rightarrow Y$ is a weak equivalence if and only if its adjoint $X \rightarrow R Y$ is a weak equivalence.

The definition of a Quillen adjoint pair can be reformulated.

Lemma 4.1.2. Let $L$ and $R$ be a pair of functors between model categories such that $L$ is left adjoint to $R$.

(1) $L$ preserves cofibrations if and only if $R$ preserves trivial fibrations.

(2) $L$ preserves trivial cofibrations if and only if $R$ preserves fibrations.

This lemma is an immediate corollary of Lemma 3.2.10; see also [DS95, 9.8]. A useful lemma associated to these questions is Ken Brown's lemma.

Lemma 4.1.3 (Ken Brown's Lemma). Let $F$ be a functor between model categories.

(1) If F takes trivial cofibrations between cofibrant objects to weak equivalences, then $F$ preserves all weak equivalences between cofibrant objects.

(2) If $F$ takes trivial fibrations between fibrant objects to weak equivalences, then $F$ preserves all weak equivalences between fibrant objects.

For the proof of this lemma, see [DS95, 9.9].

In particular, a left Quillen functor $L$ preserves weak equivalences between cofibrant objects, and a right Quillen functor $R$ preserves weak equivalences between fibrant objects.

The following proposition is the reason Quillen equivalences are important.

Proposition 4.1.4. A Quillen adjoint pair of functors between model categories induces an adjoint pair of functors on the homotopy categories which is an adjoint equivalence if and only if the adjoint pair of functors is a Quillen equivalence.

For the proof of this proposition, see [DS95, Theorem 9.7].

We now describe a useful sufficient condition for a Quillen adjoint pair to be a Quillen equivalence. 
Definition 4.1.5. Suppose $F: \mathcal{C} \rightarrow \mathcal{D}$ is a functor between model categories. For any full subcategory $\mathcal{C}^{\prime}$ of $\mathcal{C}$, we say that $F$ detects and preserves weak equivalences of $\mathrm{C}^{\prime}$ if a map $f$ in $\mathrm{C}^{\prime}$ is a weak equivalence if and only if $F f$ is.

In practice, very few functors detect and preserve weak equivalences on the whole category. However, many functors detect and preserve weak equivalences between cofibrant objects or fibrant objects, so the next lemma is often useful. Before stating it, we need a definition.

Definition 4.1.6. Suppose $\mathcal{C}$ is a model category. A fibrant replacement functor on $\mathcal{C}$ is a functor $K: \mathcal{C} \rightarrow \mathcal{C}$ whose image lies in the full subcategory of fibrant objects, together with a natural weak equivalence $i: X \rightarrow K X$.

There is a dual notion of a cofibrant replacement functor, but we do not use it. Fibrant replacement functors are usually obtained by using a version of the Factorization Lemma 3.2.11 appropriate for $\mathcal{C}$ to functorially factor the map $X \rightarrow 1$ into a trivial cofibration followed by a fibration. We have already used fibrant replacement functors in $\mathcal{S}_{*}$ in the proof of Theorem 3.1.11.

Lemma 4.1.7. Suppose $L: \mathcal{C} \rightarrow \mathcal{D}$ is a left Quillen functor with right adjoint $R$, and suppose $K$ is a fibrant replacement functor on $\mathcal{D}$. Suppose $R$ detects and preserves weak equivalences between fibrant objects and the composition $X \rightarrow R L X \stackrel{R i}{\longrightarrow}$ $R K L X$ is a weak equivalence for all cofibrant objects $X$ of $\mathcal{C}$. Then the pair $(L, R)$ is a Quillen equivalence.

There is also a dual statement, but this is the criterion we use.

Proof. Suppose $f: L X \rightarrow Y$ is a map, where $X$ is cofibrant and $Y$ is fibrant. Consider the commutative diagram below:

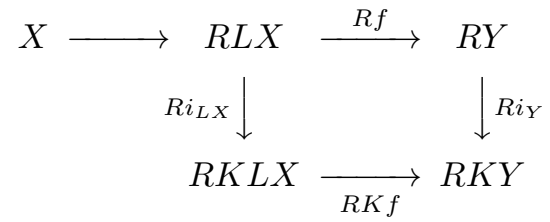

The top composite is the adjoint $g: X \rightarrow R Y$ of $f$. The map $i_{Y}$ is a weak equivalence between fibrant objects, so $R i_{Y}$ is a weak equivalence. The composite $X \rightarrow R L X \stackrel{R i_{L X}}{\longrightarrow} R K L X$ is a weak equivalence by hypothesis. Thus $g$ is a weak equivalence if and only if $R K f$ is a weak equivalence. But $R$ detects and preserves weak equivalences between fibrant objects, so $R K f$ is a weak equivalence if and only if $K f$ is a weak equivalence. Since $i$ is a natural weak equivalence, $K f$ is a weak equivalence if and only if $f$ is a weak equivalence.

4.2. The Quillen equivalence. In this section we first recall from [BF78] the stable homotopy theory of (non-symmetric) spectra. The goal of this section is to show that the forgetful functor $U$ from symmetric spectra to spectra is part of a Quillen equivalence. Obviously this requires that $U$ have a left adjoint $V: S p^{\mathbb{N}} \rightarrow S p^{\Sigma}$. We will assume the existence of $V$ in this section, and construct $V$ in Section 4.3.

Definition 4.2.1. Suppose $f: X \rightarrow Y$ is a map of spectra.

(1) The map $f$ is a stable equivalence if $\pi_{*} f$ is an isomorphism where $\pi_{k} X=$ $\operatorname{colim}_{n} \pi_{k+n} X_{n}$. 
(2) The map $f$ is a stable cofibration if $f_{0}: X_{0} \rightarrow Y_{0}$ is a monomorphism and the induced map $X_{n} \amalg_{X_{n-1} \wedge S^{1}}\left(Y_{n-1} \wedge S^{1}\right) \rightarrow Y_{n}$ is a monomorphism for all $n>0$.

(3) The map $f$ is a stable fibration if $f$ is a level fibration and

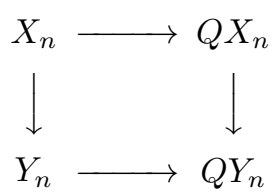

is a homotopy pullback square for each $n$.

Theorem 4.2.2 ([BF78]). The stable equivalences, stable cofibrations, and stable fibrations define a model structure on $S p^{\mathbb{N}}$.

Before turning to the Quillen equivalence we need the following proposition.

Proposition 4.2.3. If a map $f: E \rightarrow B$ in $S p^{\mathbb{N}}$ is a level fibration and, for all $n \geq 0$, the diagram

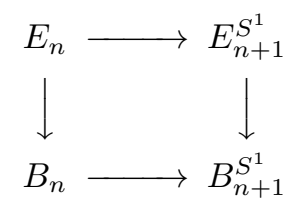

is a homotopy pullback square, then $f$ is a stable fibration.

The most elegant way to prove this proposition is to follow the development of Section 3 for spectra. Theorem 3.1.11 becomes stronger in this situation; we find that stable equivalences coincide with stable homotopy isomorphisms. The above proposition is then the analogue of Lemma 3.4.12.

Proposition 4.2.4. The functors $U: S p^{\Sigma} \rightarrow S p^{\mathbb{N}}$ and its left adjoint $V$ are a Quillen adjoint pair.

Proof. Proposition 4.2.3 implies that $U$ preserves stable fibrations. The stable trivial fibrations in $S p^{\Sigma}$ and in $S p^{\mathbb{N}}$ are the level trivial fibrations; see [BF78, A.8 ii]. So $U$ preserves stable trivial fibrations as well.

Theorem 4.2.5. The functor $U: S p^{\Sigma} \rightarrow S p^{\mathbb{N}}$ and its left adjoint $V$ form a Quillen equivalence of the stable model categories.

We prove this theorem by using Lemma 4.1.7. In particular, we need to understand stable equivalences between stably fibrant objects.

Lemma 4.2.6. Suppose $f: X \rightarrow Y$ is a stable equivalence between stably fibrant objects in either $S p^{\Sigma}$ or $S p^{\mathbb{N}}$. Then $f$ is a level equivalence.

Proof. Factor $f$ as a stably trivial cofibration, $i$, followed by a stable fibration, $p$. Since $f$ is a stable equivalence, $p$ is also. Hence, $p$ is a level trivial fibration by Lemma 3.4.5. Also, $i$ is a stably trivial cofibration between stably fibrant objects, hence it is a strong deformation retract; see [Qui67, II p. 2.5]. To see this, note that $i$ has the left lifting property with respect to $X \rightarrow *$, so the lift constructs a homotopy inverse to $i$. Because the simplicial structure is given on levels, a strong deformation retract here is a level equivalence. So both $i$ and $p$ are level equivalences, hence so is $f$. 
Corollary 4.2.7. $U: S p^{\Sigma} \rightarrow S p^{\mathbb{N}}$ detects and preserves stable equivalences between stably fibrant objects.

Let $L$ denote a fibrant replacement functor in $S p^{\Sigma}$, obtained by factoring $X \rightarrow *$ into a stable trivial cofibration followed by a stable fibration. By Lemma 4.1.7 and Corollary 4.2.7, to prove Theorem 4.2.5 it suffices to show that $X \rightarrow U L V X$ is a stable equivalence for all cofibrant (non-symmetric) spectra $X$. We prove this in several steps.

Definition 4.2.8. Given a simplicial set $X$, define $\widetilde{F}_{n}(X)$ to be the (non-symmetric) spectrum whose $m$ th level is $S^{m-n} \wedge X$ for $m \geq n$ and the basepoint otherwise, with the obvious structure maps. This defines a functor $\widetilde{F}_{n}: \mathcal{S}_{*} \rightarrow S p^{\mathbb{N}}$ left adjoint to the evaluation functor $\mathrm{Ev}_{n}$.

Note that $\widetilde{F}_{0} X=\Sigma^{\infty} X$. Also, since $U \circ \mathrm{Ev}_{n}=\mathrm{Ev}_{n}$, the left adjoints satisfy $V \circ \widetilde{F}_{n}=F_{n}$.

Lemma 4.2.9. The map $X \rightarrow U L V X$ is a stable equivalence when $X=\Sigma^{\infty} Y=$ $\widetilde{F}_{0} Y$ for any $Y \in \mathcal{S}_{*}$.

Proof. Consider the functor on simplicial sets $Q Z=\operatorname{colim} \Omega^{n} K \Sigma^{n} Z$, where $K$ is a simplicial fibrant replacement functor. Because $Q$ is simplicial we can prolong it to a functor on $S p^{\Sigma}$. The map $F_{0} Y \rightarrow Q F_{0} Y$ induces an isomorphism on stable homotopy. Also $Q F_{0} Y$ is an $\Omega$-spectrum since $Q Z \rightarrow \Omega Q \Sigma Z$ is a weak equivalence for any $Z \in \mathcal{S}_{*}$. Hence $Q F_{0} Y$ is level equivalent to $L F_{0} Y$, so $F_{0} Y \rightarrow L F_{0} Y$ induces an isomorphism in stable homotopy. Since $\widetilde{F}_{0} Y \rightarrow U F_{0} Y$ is a level equivalence and $U F_{0} Y \rightarrow U L F_{0} Y$ is a stable homotopy equivalence, the lemma follows.

Because both $S p^{\Sigma}$ and $S p^{\mathbb{N}}$ are stable model categories, the following lemma is expected.

Lemma 4.2.10. Suppose $X$ is a cofibrant spectrum in $S p^{\mathbb{N}}$. Then the map $X \rightarrow$ $U L V X$ is a stable equivalence if and only if $X \wedge S^{1} \rightarrow U L V\left(X \wedge S^{1}\right)$ is a stable equivalence.

Proof. For notational convenience, we write $\Sigma X$ for $X \wedge S^{1}$ and $\Omega X$ for $X^{S^{1}}$ in this proof, for $X$ a (possibly symmetric) spectrum. Consider the stable trivial cofibration $\Sigma V X \rightarrow L \Sigma V X$ in $S p^{\Sigma}$. By Theorem 3.1.14, part three, $V X \rightarrow \Omega L \Sigma V X$ is also a stable equivalence. By the lifting property of the stable trivial cofibration $V X \rightarrow L V X$ and the 2-out-of-3 property, there is a stable equivalence $L V X \rightarrow \Omega L \Sigma V X$. This map is a stable equivalence between stably fibrant objects, so by Corollary 4.2.7, $f: U L V X \rightarrow U \Omega L \Sigma V X$ is a stable equivalence.

So $g: X \rightarrow U L V X$ is a stable equivalence if and only if $f g: X \rightarrow U \Omega L \Sigma V X$ is a stable equivalence. Since $\Omega$ and $U$ commute, $g f$ is a stable equivalence if and only if $\Sigma X \rightarrow U L \Sigma V X$ is a stable equivalence by part three of Theorem 3.1.14 for (non-symmetric) spectra. But, since $U$ commutes with $\Omega$, the left adjoint $V$ commutes with $\Sigma$, so we have a natural isomorphism $U L \Sigma V X \rightarrow U L V \Sigma X$. This completes the proof.

Lemma 4.2.11. Let $f: X \rightarrow Y$ be a stable equivalence between cofibrant spectra in $S p^{\mathbb{N}}$. Then $X \rightarrow U L V X$ is a stable equivalence if and only if $Y \rightarrow U L V Y$ is a stable equivalence. 
Proof. Consider the following commuting square:

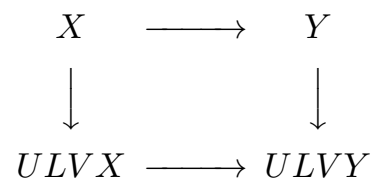

Since $V$ is a left Quillen functor by Proposition 4.2.4, it preserves trivial cofibrations. Hence, by Ken Brown's Lemma 4.1.3, $V$ preserves stable equivalences between cofibrant objects. Hence $V X \rightarrow V Y$ is a stable equivalence. $L$ takes stable equivalences to level equivalences, by Lemma 4.2.6. So $U L V X \rightarrow U L V Y$ is a level equivalence since $U$ preserves level equivalences. Hence the top and bottom maps are stable equivalences, so the right map is a stable equivalence if and only if the left map is.

Using the preceding three lemmas we can extend Lemma 4.2.9 to any cofibrant strictly bounded below spectrum.

Definition 4.2.12. Define a spectrum $X \in S p^{\mathbb{N}}$ to be strictly bounded below if there is an $n$ such that for all $m \geq n$ the structure map $S^{1} \wedge X_{m} \rightarrow X_{m+1}$ is an isomorphism.

Lemma 4.2.13. Suppose $X \in S p^{\mathbb{N}}$ is cofibrant and strictly bounded below. Then the map $X \rightarrow U L V X$ is a stable equivalence.

Proof. Suppose $X$ is strictly bounded below at $n$. Then we have a map $\widetilde{F}_{n} X_{n} \stackrel{g}{\rightarrow} X$ which is the identity on all levels $\geq n$. In particular, $g$ is a stable homotopy equivalence. Applying Lemma 4.2.11, this shows that to prove the lemma it is enough to show that $\widetilde{F}_{n} X_{n} \rightarrow U L F_{n} X_{n}$ is a stable equivalence. But there is an evident map $\widetilde{F}_{n} X_{n} \wedge S^{n} \cong \widetilde{F}_{n}\left(X_{n} \wedge S^{n}\right) \rightarrow \Sigma^{\infty} X_{n}$ which is the identity map above level $n-1$, and so is a stable equivalence. Lemmas 4.2.9, 4.2.10, and 4.2.11 complete the proof.

We now extend this lemma to all cofibrant objects, completing the proof of Theorem 4.2.5. First, we need to recall a basic fact about simplicial sets. Recall that the homotopy group $\pi_{n} X$ of a pointed simplicial set $X$ is defined to be $\pi_{0} \operatorname{Map}_{\mathcal{S}_{*}}\left(S^{n}, K X\right)$, where $K$ is a fibrant replacement functor. This ensures that weak equivalences are homotopy isomorphisms. If $X$ is already a Kan complex, $X$ is simplicially homotopy equivalent to $K X$, and so $\pi_{n} X \cong \pi_{0} \operatorname{Map}_{\mathcal{S}_{*}}\left(S^{n}, X\right)$. Since the simplicial sets $\partial \Delta[n]_{+}$and $\Delta[n]_{+}$are finite, the colimit of a sequence of Kan complexes is again a Kan complex. Since the simplicial sets $S^{n}$ and $S^{n} \wedge \Delta[1]_{+}$ are finite, homotopy commutes with filtered colimits of Kan complexes, and in particular with transfinite compositions of maps of Kan complexes.

In fact, homotopy commutes with transfinite compositions of arbitrary monomorphisms of simplicial sets. To see this, apply the geometric realization to get a sequence of cofibrations of CW complexes. Since homotopy commutes with such transfinite compositions, the result follows.

Lemma 4.2.14. Suppose $X$ is a cofibrant object of $S p^{\mathbb{N}}$. Then the map $X \rightarrow$ $U L V X$ is a stable equivalence.

Proof. Let $X^{i}$ denote the truncation of $X$ at $i$. That is, we have $X_{n}^{i}=X_{n}$ for $n \leq i$ and $X_{n}^{i}=X_{i} \wedge S^{n-i}$ for $n \geq i$. Then the $X^{i}$ are strictly bounded below and 
cofibrant, and there are monomorphisms $X^{i} \rightarrow X^{i+1}$ with $\operatorname{colim}_{i} X^{i}=X$. Thus each map $X^{i} \rightarrow U L V X^{i}$ is a stable equivalence.

We claim that the induced map $X \rightarrow \operatorname{colim}_{i} U L V X^{i}$ is a stable equivalence. To see this, note that

$$
\pi_{n} X=\operatorname{colim}_{i} \pi_{n+i} X_{i}=\operatorname{colim}_{i} \pi_{n+i} \operatorname{colim}_{j} X_{i}^{j} .
$$

Since homotopy commutes with transfinite compositions of monomorphisms, we find that $\pi_{n} X \cong \operatorname{colim}_{j} \pi_{n} X^{j}$. Similarly, since homotopy of Kan complexes commutes with arbitrary filtered colimits, we find $\pi_{n} \operatorname{colim}_{i} U L V X^{i} \cong \operatorname{colim}_{i} \pi_{n} U L V X^{i}$. It follows that $X \rightarrow \operatorname{colim}_{i} U L V X^{i}$ is a stable homotopy equivalence, as required.

We now examine the relationship between $U L V X$ and $\operatorname{colim}_{i} U L V X^{i}$. Since $V$ is a left adjoint, $V X \cong \operatorname{colim}_{i} V X^{i}$. Each map $V X^{i} \rightarrow L V X^{i}$ is a stable trivial cofibration; by Lemma 4.2 .15 below then $\operatorname{colim}_{i} V X^{i} \rightarrow \operatorname{colim}_{i} L V X^{i}$ is a stable equivalence.

We now claim that $\operatorname{colim}_{i} L V X^{i}$ is an $\Omega$-spectrum, and thus is stably fibrant. Indeed, $\operatorname{colim}_{i} L V X^{i}$ is a level Kan complex by the comments preceding this lemma. Similarly, $\left(\operatorname{colim} L V X^{i}\right)_{n+1}^{S^{1}}=\operatorname{colim}_{i}\left(\left(L V X^{i}\right)_{n+1}^{S^{1}}\right)$. Since homotopy of Kan complexes commutes with filtered colimits, it follows that $\operatorname{colim}_{i} L V X^{i}$ is an $\Omega$ spectrum.

Hence the stable equivalence $V X \rightarrow \operatorname{colim}_{i} L V X^{i}$ extends to a stable equivalence $L V X \rightarrow \operatorname{colim}_{i} L V X^{i}$. By Lemma 4.2.6, this map is actually a level equivalence. Since $U$ preserves level equivalences and colimits, the map $U L V X \rightarrow$ $\operatorname{colim}_{i} U L V X^{i}$ is also a level equivalence. We have seen above that the map $X \rightarrow \operatorname{colim}_{i} U L V X^{i}$ is a stable equivalence, so $X \rightarrow U L V X$ must also be a stable equivalence.

Lemma 4.2.15. Suppose given two sequential colimits in $S p^{\Sigma}, A=\operatorname{colim}_{i} A^{i}$ and $B=\operatorname{colim}_{i} B^{i}$ with each $B^{i}$ level fibrant and commuting maps $f^{i}: A^{i} \rightarrow B^{i}$ which are stable equivalences. Then $\operatorname{colim}_{i} f^{i}: A \rightarrow B$ is a stable equivalence.

Proof. We inductively define a new sequence $C_{i}$ and maps $A_{i} \rightarrow C_{i}$ and $C_{i} \rightarrow B_{i}$. Define $C_{0}=A_{0}$. Having defined $C_{i}$, define $C_{i+1}$ by factoring the map $C_{i} \amalg_{A_{i} i}$ $A_{i+1} \rightarrow B_{i+1}$ into a stable trivial cofibration to $C_{i+1}$ followed by a stable fibration $C_{i+1} \rightarrow B_{i+1}$. Then the induced map $\operatorname{colim}_{i} A_{i} \rightarrow \operatorname{colim}_{i} C_{i}$ is a stable trivial cofibration, by a lifting argument. On the other hand, each map $C_{i} \rightarrow B_{i}$ is a stable equivalence, by the two-out-of-three axiom. In fact, the maps $C_{i} \rightarrow B_{i}$ are stable trivial fibrations, and hence level equivalences. Since homotopy of level Kan complexes commutes with filtered colimits, we find that $\operatorname{colim} C_{i} \rightarrow \operatorname{colim}_{i} B_{i}$ is a level equivalence, and therefore that $A=\operatorname{colim}_{i} A_{i} \rightarrow \operatorname{colim}_{i} B_{i}=B$ is a stable equivalence.

Remark 4.2.16. It follows from the results of Section 5.3 that the smash product on $S p^{\Sigma}$ induces a smash product on $\operatorname{Ho} S p^{\Sigma}$. The handicrafted smash products of [Ada74] induce a smash product on Ho $S p^{\mathbb{N}}$. We now consider to what extent the equivalence $R U:$ Ho $S p^{\Sigma} \rightarrow$ Ho $S p^{\mathbb{N}}$ induced by $U$ preserves these smash products. Since $U$ is a simplicial functor, there is a natural isomorphism $R U(X \wedge Y) \cong$ $(R U)(X) \wedge(R U)(Y)$ for all (arbitrary desuspensions of) suspension spectra $X$. On the other hand, in either Ho $S p^{\Sigma}$ or Ho $S p^{\mathbb{N}}, X \wedge Y$ is determined by the collection of $F \wedge Y$ for all finite spectra $F$ mapping to $X$. To be precise, $X \wedge Y$ is the minimal weak colimit [HPS97] of the $F \wedge Y$. As an equivalence of categories, $R U$ preserves minimal 
weak colimits, so there is an isomorphism $R U(X \wedge Y) \cong(R U)(X) \wedge(R U)(Y)$. However, we do not know if this is natural, as the minimal weak colimit is only a weak colimit. This isomorphism is natural up to phantom maps, however.

4.3. Description of $V$. This short section is devoted to the construction of the left adjoint $V: S p^{\mathbb{N}} \rightarrow S p^{\Sigma}$ to the forgetful functor $U: S p^{\Sigma} \rightarrow S p^{\mathbb{N}}$.

Recall that, in any cocomplete symmetric monoidal category $\mathcal{C}$, the free monoid or tensor algebra generated by an object $X$ is $T(X)=e \vee X \vee X^{\otimes 2} \vee \cdots \vee X^{\otimes n} \vee \cdots$, where $e$ is the unit and $\vee$ is the coproduct. The multiplication on $T(X)$ is the concatenation $X^{\otimes n} \otimes X^{\otimes m} \rightarrow X^{\otimes(n+m)}$. Similarly, the free commutative monoid on an object $X$ is $\operatorname{Sym}(X)=e \vee X \vee\left(X^{\otimes 2} / \Sigma_{2}\right) \vee \cdots \vee\left(X^{\otimes n} / \Sigma_{n}\right) \vee \cdots$.

Recall that the evaluation functor $\mathrm{Ev}_{n}: \mathcal{S}_{*}^{\Sigma} \rightarrow \mathcal{S}_{*}$ has a left adjoint $G_{n}$, where $G_{n} X$ is $\left(\Sigma_{n}\right)_{+} \wedge X$ at level $n$ and the basepoint everywhere else. Similarly, the evaluation functor $\mathrm{Ev}_{n}: \mathcal{S}_{*}^{\mathbb{N}} \rightarrow \mathcal{S}_{*}$ has a left adjoint $\widetilde{G}_{n}$, where $\widetilde{G}_{n}$ is $X$ at level $n$ and the basepoint everywhere else.

Lemma 4.3.1. In the category $\mathcal{S}_{*}^{\mathbb{N}}$ of sequences, the sphere spectrum $S$ is the tensor algebra on the sequence $\widetilde{G}_{1} S^{1}=\left(*, S^{1}, *, \ldots, *, \ldots\right)$. In the category $\mathcal{S}_{*}^{\Sigma}$ of symmetric sequences, the sphere symmetric spectrum $S$ is the free commutative monoid on the symmetric sequence $G_{1} S^{1}=\left(*, S^{1}, *, \ldots, *, \ldots\right)$.

Proof. The first statement follows directly from the definitions. In the category of symmetric sequences, $\left(G_{1} S^{1}\right)^{\otimes n}=G_{n} S^{n}$, so $T\left(G_{1} S^{1}\right)$ is $\left(\Sigma_{n}\right)_{+} \wedge S^{n}$ in degree $n$. Therefore $\operatorname{Sym}\left(G_{1} S^{1}\right)$ is $S^{n}$ in degree $n$. Since we already know $S$ is a commutative monoid, the map $G_{1} S^{1} \rightarrow S$ induces a map $\operatorname{Sym}\left(G_{1} S^{1}\right) \rightarrow S$ which is an isomorphism.

This lemma explains why left $S$-modules and right $S$-modules are equivalent in the category of sequences, since this is true for any tensor algebra. This lemma also explains why Remark 1.2.3 holds, since an analogous statement holds for any free commutative monoid.

Now, the forgetful functor $U: \mathcal{S}_{*}^{\Sigma} \rightarrow \mathcal{S}_{*}^{\mathbb{N}}$ has a left adjoint $G$, defined by $G X=$ $\bigvee G_{n} X_{n}$, so that the $n$th level of $G X$ is just $\left(\Sigma_{n}\right)_{+} \wedge X_{n}$. The functor $G$ is monoidal; that is, there is a natural isomorphism $G(X) \otimes G(Y) \rightarrow G(X \otimes Y)$ compatible with the associativity and unit isomorphisms. However, $G$ is definitely not a symmetric monoidal functor; this natural isomorphism is not compatible with the commutativity isomorphisms. This explains how $S$ can be commutative in $\mathcal{S}_{*}^{\Sigma}$ yet $U S=S$ is not commutative in $\mathcal{S}_{*}^{\mathbb{N}}$.

Since $G$ is a monoidal functor, $G$ preserves monoids and modules, and so defines a functor $G: S p^{\mathbb{N}} \rightarrow T\left(G_{1} S^{1}\right)$-mod, left adjoint to the forgetful functor $T\left(G_{1} S^{1}\right)$-mod $\rightarrow S p^{\mathbb{N}}$. On the other hand, the map of monoids $T\left(G_{1} S^{1}\right) \stackrel{p}{\rightarrow}$ $\operatorname{Sym}\left(G_{1} S^{1}\right)=S$ defines the usual adjoint pair of induction and restriction. Induction takes a (left) $T\left(G_{1} S^{1}\right)$-module $X$ to $S \otimes_{T\left(G_{1} S^{1}\right)} X$, where the tensor product uses the right action of $T\left(G_{1} S^{1}\right)$ on $S$ determined by $p$. It follows that the left adjoint $V: S p^{\mathbb{N}} \rightarrow S p^{\Sigma}$ of the forgetful functor $U: S p^{\Sigma} \rightarrow S p^{\mathbb{N}}$ is $V(X)=$ $S \otimes_{T\left(G_{1} S^{1}\right)} G X$.

\section{Additional properties of symmetric spectra}

In this section we discuss some properties of the category of symmetric spectra. In Section 5.1, we consider the level model structures on $S p^{\Sigma}$. In particular, we 
show that every symmetric spectrum embeds in an injective spectrum by a level equivalence, completing the proof that the stable structures define a model structure on $S p^{\Sigma}$. In Section 5.2 we characterize the stable cofibrations. In Sections 5.3 and 5.4, we study the relationship between the stable model structure on $S p^{\Sigma}$ and the smash product. This is necessary for constructing model categories of monoids, algebras, and modules, as is done in [SS97]. In Section 5.5, we show that the stable model structure on $S p^{\Sigma}$ is proper. Finally, in Section 5.6 we define semistable spectra and investigate their relationship to stable homotopy equivalences.

5.1. Level model structure. In this section we construct the two level model structures on the category of symmetric spectra.

Definition 5.1.1. A projective cofibration of symmetric spectra is a map that has the left lifting property with respect to every level trivial fibration. The projective cofibrations are the stable cofibrations from Section 3.4. The projective level structure on $S p^{\Sigma}$ is the class of level equivalences, the class of projective cofibrations, and the class of level fibrations. An injective fibration of symmetric spectra is a map that has the right lifting property with respect to every level trivial cofibration (the adjective "injective" refers to the lifting properties of the map and not to its being a monomorphism). The injective level structure is the class of level equivalences, the class of level cofibrations, and the class of injective fibrations.

Theorem 5.1.2. The projective level structure and the injective level structure are model structures on the category of symmetric spectra.

Proof. The category of symmetric spectra is bicomplete. The class of level equivalences has the two-out-of-three property. The retract axiom holds by construction in both the projective and injective level structures.

We now prove the lifting and factorization axioms, beginning with the projective level structure. We use the sets of maps $F I_{\partial}$ and $F I_{\Lambda}$ defined in Definition 3.3.2. The lifting axiom for a projective cofibration and a level trivial fibration holds by definition. The other lifting and factorization axioms follow by identifying the respective classes in terms of $F I_{\partial}$ and $F I_{\Lambda}$. By part (4) of Lemma 5.1.4, an $F I_{\Lambda^{-}}$ cofibration is a projective cofibration which is a level equivalence and an $F I_{\Lambda^{-}}$ injective map is a level fibration. Since ( $J$-cof, $J$-inj) has the lifting property for any class $J$, the lifting axiom for a map that is both a level equivalence and a projective cofibration and a map that is a level fibration follows by setting $J=F I_{\Lambda}$. Moreover, every map can be factored as the composition of an $F I_{\Lambda}$-cofibration and an $F I_{\Lambda}$-injective map. Similarly, every map can be factored as the composition of an $F I_{\partial}$-cofibration and an $F I_{\partial}$-injective map, by Lemma 3.2.11. By part (3) of

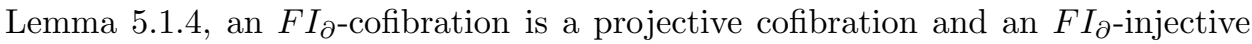
map is a level trivial fibration.

Now consider lifting and factorization for the injective level model structure. Here we use a set $C$ containing a map from each isomorphism class of monomorphisms $i: X \rightarrow Y$ with $Y$ a countable symmetric spectrum, and a set $t C$ containing a map from each isomorphism class of level trivial cofibrations $i: X \rightarrow Y$ with $Y$ a countable symmetric spectrum. The lifting axiom for a level trivial cofibration $i$ and an injective fibration $p$ holds by definition. By part (5) of Lemma 5.1.4, a $C$ cofibration is a level cofibration and a $C$-injective map is an injective fibration that is a level equivalence. Since $(J$-cof, $J$-inj) has the lifting property for any class $J$, the lifting axiom for a level cofibration and a map that is both an injective fibration 
and a level equivalence follows with $J=C$. Also, every map can be factored as the composition of a $C$-cofibration followed by a $C$-injective map, by Lemma 3.2.11. Similarly, every map can be factored as the composition of a $t C$-cofibration and a $t C$-injective map. By part (6) of Lemma 5.1.4, a $t C$-cofibration is a level trivial cofibration and a $t C$-injective map is an injective fibration.

Corollary 5.1.3. Every symmetric spectrum embeds in an injective spectrum by a map that is a level equivalence.

Proof. For a symmetric spectrum $X$, the map $X \rightarrow *$ is the composition of a level trivial cofibration $X \rightarrow E$ and an injective fibration $E \rightarrow *$. The fibrant object $E$ is an injective spectrum.

Some parts of the next lemma have already been proved. They are repeated for easy reference. Recall that $R_{n}: \mathcal{S}_{*} \rightarrow S p^{\Sigma}$ is the right adjoint of the evaluation functor $\mathrm{Ev}_{n}: S p^{\Sigma} \rightarrow \mathcal{S}_{*}$.

Lemma 5.1.4. (1) Let $K \subseteq \mathcal{S}_{*}$ be the class of $K$ an fibrations and let $R K=$ $\bigcup_{n} R_{n} K$. Then a map is RK-projective if and only if it is a level trivial cofibration.

(2) Let $t K \subseteq \mathcal{S}_{*}$ be the class of trivial Kan fibrations and let $R(t K)=\bigcup_{n} R_{n}(t K)$. Then a map is $R(t K)$-projective if and only if it is a level cofibration.

(3) Let $F I_{\partial}$ be the set defined in 3.3.2. Then a map is $F I_{\partial}$-injective if and only if it is a level trivial fibration. A map is an $F I_{\partial}$-cofibration if and only if it is a projective cofibration.

(4) Let $F I_{\Lambda}$ be the set defined in 3.3.2. Then a map is $F I_{\Lambda}$-injective if and only if it is a level fibration. A map is an $F I_{\Lambda}$-cofibration if and only if it is a projective cofibration and a level equivalence.

(5) Let $C$ be a set containing a map from each isomorphism class of monomorphisms $i: X \rightarrow Y$ with $Y$ a countable symmetric spectrum. Then a map is $C$-injective if and only if it is an injective fibration and a level equivalence. A map is a $C$-cofibration if and only if it is a level cofibration.

(6) Let $t C$ be a set containing a map from each isomorphism class of level trivial cofibrations $i: X \rightarrow Y$ with $Y$ a countable symmetric spectrum. Then a map is $t C$-injective if and only if it is an injective fibration. $A$ map is a $t C$-cofibration if and only if it is a level trivial cofibration.

Proof. Parts (1) and (2): By adjunction, a map $g$ has the left lifting property with respect to the class $R K(R(t K))$ if and only if for each $n \geq 0$ the map $\mathrm{Ev}_{n} g$ has the left lifting property with respect to $K(t K)$. But $\mathrm{Ev}_{n} g$ has the left lifting property with respect to $K(t K)$ if and only if $\mathrm{Ev}_{n} g$ is a trivial cofibration (arbitrary cofibration), i.e., if and only if $g$ is a level trivial cofibration (arbitrary level cofibration).

Part (3) is proved in Propositions 3.3.3 and 3.4.2.

Part (4): The first claim is proved in Proposition 3.3.3. Every $F I_{\Lambda}$-cofibration has the left lifting property with respect to level fibrations, so is in particular a projective cofibration by part (3). Every map in $F I_{\Lambda}$ is a level trivial cofibration by Proposition 2.2 .7 , so is $R K$-projective by part (1). So every $F I_{\Lambda}$-cofibration is also $R K$-projective and hence is a level trivial cofibration by part (1) again. So in particular it is a level equivalence.

Conversely, suppose $f$ is a projective cofibration and a level equivalence. We can factor $f$ as the composition of an $F I_{\Lambda}$-cofibration $i$ and an $F I_{\Lambda}$-injective map $p$, by 
Lemma 3.2.11. By the two-out-of-three property, $p$ is a level equivalence. Therefore the projective cofibration $f$ has the left lifting property with respect to the level trivial fibration $p$. By the Retract Argument 3.2.4, $f$ is a retract of $i$, and so is an $F I_{\Lambda}$-cofibration.

For part (5), first note that, by part (2), every $C$-cofibration is a level cofibration. Conversely, suppose $f: X \rightarrow Y$ is a level cofibration. Then $f$ is a $C$-cofibration if, for every $C$-injective map $g$ and commutative square

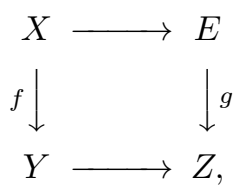

there is a lift $h: Y \rightarrow E$ making the diagram commute. Let $\mathcal{P}$ be the partially ordered set of partial lifts: an object of $\mathcal{P}$ is a pair $\left(U, h_{U}\right)$ such that $X \subseteq U \subseteq Y$ and the diagram

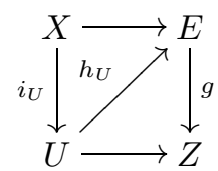

is commutative. We define $\left(U, h_{U}\right) \leq\left(V, h_{V}\right)$ if $U \subseteq V$ and $h_{V}$ extends $h_{U}$. Every chain in $\mathcal{P}$ has an upper bound and so Zorn's lemma gives a maximum $\left(M, h_{M}\right)$. Suppose $M$ is strictly contained in $Y$. Then, by taking the subspectrum generated by a simplex not in $M$, we find a countable subspectrum $D$ (by Lemma 5.1.6 below) such that the level cofibration $D \cap M \rightarrow D$ is not an isomorphism. By construction, the map $D \cap M \rightarrow D$ is isomorphic to a map in $C$. By cobase change, $M \rightarrow D \cup M$ is a $C$-cofibration. Thus $h_{M}$ extends to a partial lift on $D \cup M$, contradicting the maximality of $\left(M, h_{M}\right)$. Therefore $M=Y$, and so $f$ is a $C$-cofibration.

We now identify $C$-inj. Since $(C$-cof)-inj $=C$-inj, every $C$-injective map has the right lifting property with respect to every monomorphism. In particular, every $C$-injective map is an injective fibration. Let $f: E \rightarrow B$ be a map having the right lifting property with respect to every monomorphism. Let $s: B \rightarrow E$ be a lift in the diagram

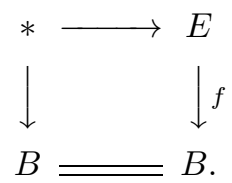

Then $f s$ is the identity map on $B$. To study the composite $s f$, let $j$ be the monomorphism $\partial \Delta[1] \rightarrow \Delta[1]$. The diagram

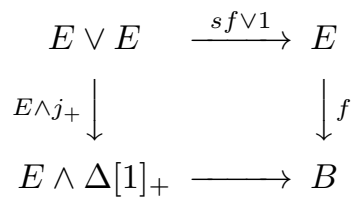

is commutative since $f s f=f$ and has a lift since $E \wedge j_{+}$is a monomorphism. The lift is a simplicial homotopy from $s f$ to the identity on $E$. Therefore $f$ is a simplicial homotopy equivalence and in particular $f$ is a level equivalence. Conversely suppose $f$ is both an injective fibration and a level equivalence. We can factor $f$ as the 
composition of a $C$-cofibration $i$ and a $C$-injective map $p$. By the two-out-of-three property, $i$ is a level equivalence. The level trivial cofibration $i$ has the left lifting property with respect to the injective fibration $f$. By the Retract Argument 3.2.4, $f$ is a retract of $p$ and so is a $C$-injective map.

The proof of part (6) is similar, though slightly more complex. By part (1), every $t C$-cofibration is a level trivial cofibration. Conversely, suppose $f: X \rightarrow Y$ is a level trivial cofibration. Then $f$ is a $t C$-cofibration if, for every $t C$-injective map $g$ and commutative square

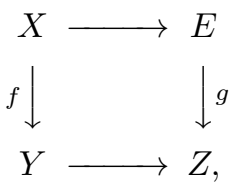

there is a lift $h: Y \rightarrow E$ making the diagram commute. We again let $\mathcal{P}$ be the partially ordered set of partial lifts: an object of $\mathcal{P}$ is a pair $\left(U, h_{U}\right)$ such that $X \subseteq U \subseteq Y$, and the diagram

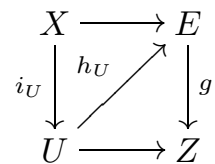

is commutative, but we also require that the inclusion $i_{U}: X \rightarrow U$ is a weak equivalence. We define $\left(U, h_{U}\right) \leq\left(V, h_{V}\right)$ as before. Every chain in $\mathcal{P}$ has an upper bound (using the fact that a transfinite composition of level trivial cofibrations is a level trivial cofibration) and so Zorn's lemma gives a maximum $\left(M, h_{M}\right)$. The inclusion $X \rightarrow M$ is a level trivial cofibration, so, by the two-out-of-three property, the inclusion $M \rightarrow Y$ is a weak equivalence. If $M$ is strictly contained in $Y$, Lemma 5.1.7, proved below, applied to the countable subspectrum of $Y$ generated by a simplex not in $M$, gives a countable subspectrum $D$ of $Y$ such that the monomorphism $D \cap M \rightarrow D$ is a weak equivalence but is not an isomorphism. By construction, $D \cap M \rightarrow D$ is isomorphic to a map in $t C$. By cobase change, $M \rightarrow D \cup M$ is a $t C$-cofibration. So $h_{M}$ extends to a partial lift on $D \cup M$. This is a contradiction since $\left(M, h_{M}\right)$ is maximal. Thus $M=Y$, and so $f$ is a $t C$-cofibration.

Since $(t C$-cof)-inj $=t C$-inj, the $t C$-injective maps are the injective fibrations.

Corollary 5.1.5. Every injective fibration is a level fibration and every projective cofibration is a level cofibration.

Proof. By Proposition 2.2.7, every map in $F I_{\partial}$ is a level cofibration. Therefore, by part (2) of Lemma 5.1.4, every projective cofibration is a level cofibration. By Proposition 2.2.7, every map in $F I_{\Lambda}$ is a level trivial cofibration. Therefore every injective fibration is a level fibration.

The following lemmas are used in the proof of Lemma 5.1.4.

Lemma 5.1.6. Let $X$ be a spectrum, and suppose $x$ is a simplex of $X_{n}$ for some $n \geq 0$. Then the smallest subspectrum of $X$ containing $x$ is countable.

Proof. First note that if $L$ is a countable collection of simplices in a simplicial set $K$, then the smallest subsimplicial set of $K$ containing $L$ is also countable. Indeed, we need only include all degeneracies of all faces of simplices in $L$, of which there are 
only countably many. Similarly, if $L$ is a countable collection of simplices in a $\Sigma_{n^{-}}$ simplicial set $K$, then the smallest sub- $\Sigma_{n}$-simplicial set containing $L$ is countable. Indeed, we only need to include the orbits of all degeneracies of all faces of simplices in $L$.

Now, let $Y_{n}$ denote the sub- $\Sigma_{n}$-simplicial set of $X_{n}$ generated by $x$. We have just seen that $Y_{n}$ is countable. We then inductively define $Y_{n+k}$ to be the smallest sub- $\Sigma_{n+k}$-simplicial set of $X_{n+k}$ containing the image of $S^{1} \wedge Y_{n+k-1}$. Then each $Y_{n+k}$ is countable, and the $Y_{n+k}$ define a subspectrum of $X$ containing $x$.

It follows in similar fashion that the smallest subspectrum of a spectrum $X$ containing any countable collection of simplices of $X$ is countable.

We need a similar lemma for inclusions which are level equivalences. To prove such a lemma, we need to recall from the comments before Lemma 4.2.14 that homotopy of simplicial sets commutes with transfinite compositions of monomorphisms. The same methods imply that relative homotopy commutes with transfinite compositions of monomorphisms.

Lemma 5.1.7. Let $f: X \rightarrow Y$ be a level trivial cofibration of symmetric spectra. For every countable subspectrum $C$ of $Y$ there is a countable subspectrum $D$ of $Y$ such that $C \subseteq D$ and $D \cap X \rightarrow D$ is a level trivial cofibration.

Proof. Let $K \subseteq L$ be a pair of pointed simplicial sets and let $v$ be a 0 -simplex of $K$. For $n \geq 1$, let $\pi_{n}(L, K ; v)$ denote the relative homotopy set of the pair with the null element as the basepoint (ignore the group structure when $n \geq 2$ ). To ease notation let $\pi_{0}(L, K ; v)$ be the pointed set $\pi_{0} L / \pi_{0} K$. The inclusion $K \rightarrow L$ is a weak equivalence if and only if $\pi_{n}(L, K ; v)=*$ for every $v \in K_{0}$ and $n \geq 0$.

Now, construct a countable spectrum $F C$ such that the map $\pi_{*}\left(C_{n}, C_{n} \cap X_{n} ; v\right) \rightarrow$ $\pi_{*}\left(F C_{n}, F C_{n} \cap X_{n} ; v\right)$ factors through the basepoint $*$ for every 0 -simplex $v$ of $C_{n} \cap X_{n}$ and integer $n \geq 0$. Since $\pi_{*}(Y, X ; v)=*$ and $\pi_{*}$ commutes with filtered colimits, for each homotopy class $\alpha \in \pi_{*}\left(C_{n}, C_{n} \cap X_{n} ; v\right)$ there is a finite simplicial subset $K_{\alpha} \subseteq Y_{n}$ such that $\pi_{*}\left(C_{n}, C_{n} \cap X_{n} ; v\right) \rightarrow \pi_{*}\left(K_{\alpha} \cup C_{n},\left(K_{\alpha} \cup C_{n}\right) \cap X_{n} ; v\right)$ sends $\alpha$ to the basepoint. Since $C_{n}$ is countable, the set $\pi_{*}\left(C_{n}, C_{n} \cap X_{n} ; v\right)$ is countable. Let $B_{n}$ be the union of $C_{n}$ with all the finite simplicial sets $K_{\alpha}$. The $B_{n}$ are countable simplicial sets and generate a countable subspectrum $F C$ of $Y$ having the desired property.

Repeat the construction to get a sequence of countable subspectra of $Y$ :

$$
C \rightarrow F C \rightarrow F^{2} C \rightarrow \cdots \rightarrow F^{n} C \rightarrow \cdots .
$$

Let $D=\operatorname{colim}_{n} F^{n} C$. The spectrum $D$ is countable. Since relative homotopy commutes with transfinite compositions of monomorphisms, $\pi_{*}\left(D_{n}, D_{n} \cap X_{n} ; v\right)$ is a set with only one element. Therefore the inclusion $D_{n} \cap X_{n} \rightarrow D_{n}$ is a weak equivalence, and so $D \cap X \rightarrow D$ is a level equivalence.

5.2. Stable cofibrations. The object of this section is to give a characterization of stable cofibrations in $S p^{\Sigma}$. To this end, we introduce the latching space.

Definition 5.2.1. Define $\bar{S}$ to be the symmetric spectrum such that $\bar{S}_{n}=S^{n}$ for $n>0$ and $\bar{S}_{0}=*$. The structure maps are the evident ones. Given a symmetric spectrum $X$, define the $n$th latching space, $L_{n} X$, to be $\operatorname{Ev}_{n}(X \wedge \bar{S})$.

There is a map of symmetric spectra $i: \bar{S} \rightarrow S$ which is the identity on positive levels. This induces a natural transformation $L_{n} X \rightarrow X_{n}$ of pointed $\Sigma_{n}$ simplicial sets. 
The following proposition uses a model structure on the category of pointed $\Sigma_{n}$ simplicial sets. A map $f: X \rightarrow Y$ of pointed $\Sigma_{n}$ simplicial sets is a $\Sigma_{n}$-fibration if it is a Kan fibration of the underlying simplicial sets. Similarly, $f$ is a weak equivalence if it is a weak equivalence of the underlying simplicial sets. The map $f$ is a $\Sigma_{n}$-cofibration if it is a monomorphism such that $\Sigma_{n}$ acts freely on the simplices of $Y$ not in the image of $f$. It is well-known, and easy to check, that the $\Sigma_{n}$-cofibrations, the $\Sigma_{n}$-fibrations, and the weak equivalences define a model structure on the category of pointed $\Sigma_{n}$-simplicial sets.

Proposition 5.2.2. A map $f: X \rightarrow Y$ in $S p^{\Sigma}$ is a stable cofibration if and only if for all $n \geq 0$ the induced map $\operatorname{Ev}_{n}(f \square i): X_{n} \amalg_{L_{n} X} L_{n} Y \rightarrow Y_{n}$ is a $\Sigma_{n}$-cofibration.

Proof. Suppose first that $\operatorname{Ev}_{n}(f \square i)$ is a $\Sigma_{n}$-cofibration for all $n$. Suppose $g: E \rightarrow B$ is a level trivial fibration. We show that $f$ has the left lifting property with respect to $g$ by constructing a lift using induction on $n$. A partial lift defines a commutative square

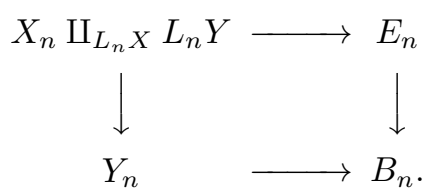

Since the left vertical map is a $\Sigma_{n}$-cofibration and the right vertical map is a trivial $\Sigma_{n}$-fibration, there is a lift in this diagram and so we can extend our partial lift. Hence $f$ has the left lifting property with respect to $g$, and so $f$ is a stable cofibration.

To prove the converse, note that $\mathrm{Ev}_{n}$ is a left adjoint as a functor to pointed $\Sigma_{n}$ simplicial sets. Since the class of stable cofibrations is the class $F I_{\partial}$-cof, it suffices to check that $\operatorname{Ev}_{n}(f \square i)$ is a $\Sigma_{n}$-cofibration for $f \in F I_{\partial}$. More generally, suppose $g: A \rightarrow B$ is a monomorphism of pointed simplicial sets. Since $F_{m} g=S \otimes G_{m} g$, we have $F_{m} g \square i=G_{m} g \square i$, where the second $\square$ is taken in $\mathcal{S}_{*}^{\Sigma}$. One can easily check that $\operatorname{Ev}_{n}\left(G_{m} g \square i\right)$ is an isomorphism when $n \neq m$ and is the map $\left(\Sigma_{n}\right)_{+} \wedge g$ when $n=m$. In both cases, $\operatorname{Ev}_{n}\left(G_{m} g \square i\right)$ is a $\Sigma_{n}$-cofibration, as required.

5.3. Pushout smash product. In this section we consider the pushout smash product in an arbitrary symmetric monoidal category and apply our results to $S p^{\Sigma}$. We show that the projective level structure and the stable model structure on $S p^{\Sigma}$ are both compatible with the symmetric monoidal structure. A monoid $E$ in $S p^{\Sigma}$ is called a symmetric ring spectrum, and is similar to an $A_{\infty}$-ring spectrum. Thus, there should be a stable model structure on the category of $E$-modules. Similarly, there should be a model structure on the category of symmetric ring spectra and the category of commutative symmetric ring spectra. These issues are dealt with more fully in [SS97] and in work in progress of the third author. Their work depends heavily on the results in this section and in Section 5.4. The results of this section alone suffice to construct a stable model structure on the category of modules over a symmetric ring spectrum which is stably cofibrant. This section also contains brief descriptions of two other stable model structures on $S p^{\Sigma}$.

Definition 5.3.1. Let $f: U \rightarrow V$ and $g: X \rightarrow Y$ be maps in a symmetric monoidal category $\mathcal{C}$. The pushout smash product

$$
f \square g: V \wedge X \amalg_{U \wedge X} U \wedge Y \rightarrow V \wedge Y
$$


is the natural map on the pushout defined by the commutative square

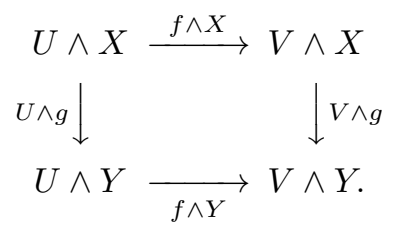

If $\mathcal{C}$ is a closed symmetric monoidal category,

$$
\operatorname{Hom}_{\square}(f, g): \operatorname{Hom}(V, X) \rightarrow \operatorname{Hom}(U, X) \times_{\operatorname{Hom}(U, Y)} \operatorname{Hom}(V, Y)
$$

is the natural map to the fiber product defined by the commutative square

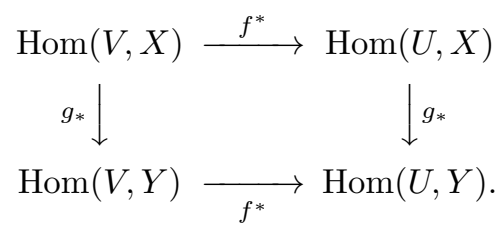

Definition 5.3.2. A model structure on a symmetric monoidal category is called monoidal if the pushout smash product $f \square g$ of two cofibrations $f$ and $g$ is a cofibration which is trivial if either $f$ or $g$ is.

In our situation, this is the correct condition to require so that the model structure is compatible with the symmetric monoidal structure. Since the unit, $S$, is cofibrant in symmetric spectra this condition also ensures that the symmetric monoidal structure induces a symmetric monoidal structure on the homotopy category. For a more general discussion of monoidal model structures, see [Hov98a].

Recall, from Definition 3.3.6, the map of sets $\mathcal{C}_{\square}(f, g)$.

Proposition 5.3.3. Let $f, g$ and $h$ be maps in a closed symmetric monoidal category $\mathrm{C}$. There is a natural isomorphism

$$
\mathcal{C}_{\square}(f \square g, h) \cong \mathcal{C}_{\square}\left(f, \operatorname{Hom}_{\square}(g, h)\right) .
$$

Proof. Use the argument in the proof of Proposition 3.3.8.

Proposition 5.3.4. Let $I$ and $J$ be classes of maps in a closed symmetric monoidal category $\mathrm{C}$. Then

$$
I \text {-cof } \square J \text {-cof } \subseteq(I \square J) \text {-cof. }
$$

Proof. Let $K=(I \square J)$-inj. By hypothesis, $(I \square J, K)$ has the lifting property. By adjunction, $\left(I, \operatorname{Hom}_{\square}(J, K)\right)$ has the lifting property. By Proposition 3.2.8, $\left(I\right.$-cof, $\left.\operatorname{Hom}_{\square}(J, K)\right)$ has the lifting property. Then $\left(J, \operatorname{Hom}_{\square}(I\right.$-cof, $\left.K)\right)$ has the lifting property, by using adjunction twice. Thus $\left(J\right.$-cof, $\operatorname{Hom}_{\square}(I$-cof, $\left.K)\right)$ has the lifting property, by Proposition 3.2.8. By adjunction, ( $I$-cof $\square J$-cof, $K$ ) has the lifting property. So $I$-cof $\square J$-cof $\subseteq(I \square J)$-cof and the proposition is proved.

Corollary 5.3.5. For classes $I, J$ and $K$ in $S p^{\Sigma}$, if $I \square J \subseteq K$-cof, then $I$-cof $\square$ $J$-cof $\subseteq K$-cof.

We now examine to what extent the pushout smash product preserves stable cofibrations and stable equivalences. To do so, we introduce a new class of maps in $S p^{\Sigma}$. 
Definition 5.3.6. Let $M$ be the class of monomorphisms in the category of symmetric sequences $\mathcal{S}_{*}^{\Sigma}$. A map of symmetric spectra is an $S$-cofibration if it is an $S \otimes M$-cofibration. A symmetric spectrum $X$ is $S$-cofibrant if the map $* \rightarrow X$ is an $S$-cofibration. A map is an $S$-fibration if it has the right lifting property with respect to every map which is both an $S$-cofibration and a stable equivalence.

Note that every stable cofibration is an $S$-cofibration, since $F I_{\partial}=S \otimes \bigcup_{n} G_{n} I_{\partial}$. On the other hand, by Proposition 2.1.9, every element of $S \otimes M$ is a monomorphism, and so every $S$-cofibration is a level cofibration. There is a model structure on $S p^{\Sigma}$, called the $S$ model structure, where the cofibrations are the $S$ cofibrations and the weak equivalences are the stable equivalences. The fibrations, called $S$-fibrations are those maps with the right lifting property with respect to $S$-cofibrations which are also stable equivalences. Every $S$-fibration is a stable fibration. This model structure will be used in a forthcoming paper by the third author to put a model structure on certain commutative $S$-algebras.

We mention as well that there is a third model structure on $S p^{\Sigma}$ where the weak equivalences are the stable equivalences, called the injective (stable) model structure. The injective cofibrations are the level cofibrations and the injective stable fibrations are all maps which are both injective fibrations and stable fibrations. In particular, the fibrant objects are the injective $\Omega$-spectra. The interested reader can prove this is a model structure using the methods of Section 3.4, replacing the set $I$ with the union of $I$ and the countable level cofibrations.

Theorem 5.3.7. Let $f$ and $g$ be maps of symmetric spectra.

(1) If $f$ and $g$ are stable cofibrations, then $f \square g$ is a stable cofibration.

(2) If $f$ and $g$ are $S$-cofibrations, then $f \square g$ is an $S$-cofibration.

(3) If $f$ is an $S$-cofibration and $g$ is a level cofibration, then $f \square g$ is a level cofibration.

(4) If $f$ is an $S$-cofibration, $g$ is a level cofibration, and either $f$ or $g$ is a level equivalence, then $f \square g$ is a level equivalence.

(5) If $f$ is an $S$-cofibration, $g$ is a level cofibration, and either $f$ or $g$ is a stable equivalence, then $f \square g$ is a stable equivalence.

Proof. Parts (1) through (4) of the proposition are proved using Corollary 5.3.5.

Part (1): Let $I=J=K=F I_{\partial}$. Then $K$-cof is the class of stable cofibrations. We have a natural isomorphism

$$
F_{p} f \square F_{q} g=F_{p+q}(f \square g)
$$

for $f, g \in \mathcal{S}_{*}$. By Proposition 1.3.4, $f \square g$ is a monomorphism when $f$ and $g$ are. Part (3) of Proposition 3.4.2 then shows that $I \square J \subseteq K$-cof. Now use the corollary.

Part (2): Let $I=J=K=S \otimes M$ (recall that $M$ is the class of monomorphisms in $\left.\mathcal{S}_{*}^{\Sigma}\right)$. By definition, $K$-cof is the class of $S$-cofibrations. For $f$ and $g$ in $\mathcal{S}_{*}^{\Sigma}$, we have a natural isomorphism

$$
S \otimes f \square S \otimes g=S \otimes(f \square g)
$$

where the first $\square$ is taken in $S p^{\Sigma}$ and the second $\square$ is taken in $\mathcal{S}_{*}^{\Sigma}$. In degree $n$,

$$
(f \square g)_{n}=\bigvee_{p+q=n}\left(\Sigma_{p+q}\right)_{+} \wedge_{\Sigma_{p} \times \Sigma_{q}}\left(f_{p} \square g_{q}\right) .
$$

For $f, g \in M$, each map $f_{p} \square g_{q}$ is a monomorphism, so it follows that $f \square g$ is a monomorphism. Thus $I \square J \subseteq K$-cof. Now use the corollary. 
Part (3): Let $I=S \otimes M$ and let $J=K$ be the class of level cofibrations. By part (5) of Lemma 5.1.4, $K$-cof $=K$. For $f \in \mathcal{S}_{*}^{\Sigma}$ and $g \in S p^{\Sigma}$, we have a natural isomorphism of maps of symmetric sequences

$$
(S \otimes f) \square g=f \square g
$$

where the first $\square$ is taken in $S p^{\Sigma}$ and the second $\square$ is taken in $\mathcal{S}_{*}^{\Sigma}$. We have seen in the proof of part (2) that $f \square g$ is a monomorphism of symmetric sequences if $f$ and $g$ are monomorphisms. Hence $I \square J \subseteq K$-cof. Now use the corollary.

Part (4): First assume $g$ is a level trivial cofibration. Let $I=S \otimes M$ and let $J=K$ be the class of level trivial cofibrations. By part (6) of Lemma 5.1.4, $K=$ $K$-cof. Proposition 1.3.4 and the method used in the proof of part (2) imply that, if $f$ and $g$ are monomorphisms of symmetric sequences and $g$ is a level equivalence, then $f \square g$ is a level equivalence. Recall that, for $h \in \mathcal{S}_{*}^{\Sigma}$ and $g \in S p^{\Sigma}$, we have a natural isomorphism of maps of symmetric sequences

$$
(S \otimes h) \square g=h \square g
$$

where the first $\square$ is taken in $S p^{\Sigma}$ and the second $\square$ is in $\mathcal{S}_{*}^{\Sigma}$. Hence $I \square J \subseteq K$-cof. Now use the corollary to prove part (4) when $g$ is a level equivalence.

It follows that, for any injective spectrum $E$ and an arbitrary $S$-cofibration $h$, the map $\operatorname{Hom}_{S}(h, E)$ is an injective fibration. Indeed, if $g$ is a level cofibration and a level equivalence, $S p^{\Sigma}\left(g, \operatorname{Hom}_{S}(h, E)\right) \cong S p^{\Sigma}(g \square h, E)$, and we have just seen that $g \square h$ is a level cofibration and a level equivalence, so $(g \square h, E)$ has the lifting property.

Now suppose $f$ is an $S$-cofibration and a level equivalence. Then the map $\operatorname{Hom}_{S p^{\Sigma}}(f, E)$ is an injective fibration and a level equivalence. Indeed, we have $\operatorname{Ev}_{k} \operatorname{Hom}_{S p^{\Sigma}}(f, E)=\operatorname{Map}_{S p^{\Sigma}}\left(f \wedge\left(S \otimes \Sigma[k]_{+}\right), E\right)$, by Remark 2.2.12. Since $S \otimes$ $\Sigma[k]_{+}$is $S$-cofibrant, and $f$ is both a level equivalence and a level cofibration, we have just proved that $f \wedge\left(S \otimes \Sigma[k]_{+}\right)=f \square\left(* \rightarrow S \otimes \Sigma[k]_{+}\right)$is a level equivalence and a level cofibration. This shows that $\pi_{0} \operatorname{Ev}_{k} \operatorname{Hom}_{S p^{\Sigma}}(f, E)$ is an isomorphism; smashing with $F_{0} S^{n}$ and using a similar argument shows that $\pi_{n} \operatorname{Ev}_{k} \operatorname{Hom}_{S p^{\Sigma}}(f, E)$ is an isomorphism.

Thus every level cofibration $g$ has the left lifting property with respect to the map $\operatorname{Hom}_{S p^{\Sigma}}(f, E)$. By adjunction, $f \square g$ and $f \square(g \square j)$, where $j: \partial \Delta[1]_{+} \rightarrow \Delta[1]_{+}$is the inclusion, have the extension property with respect to every injective spectrum $E$. It follows that $E^{0}(f \square g)$ is an isomorphism for every injective spectrum $E$ and hence that $f \square g$ is a level equivalence.

Part (5): Because we are working in a stable situation, a level cofibration $i: X \rightarrow$ $Y$ is a stable equivalence if and only if its cofiber $C_{i}=Y / X$ is stably trivial. The map $f \square g$ is a level cofibration by part (3). By commuting colimits, the cofiber of $f \square g$ is the smash product $C f \wedge C g$ of the cofiber $C f$ of $f$, which is $S$-cofibrant, and the cofiber $C g$ of $g$. Let $E$ be an injective $\Omega$-spectrum. We will show that $\operatorname{Hom}_{S}(C f \wedge C g, E)$ is a level trivial spectrum, and thus that $C f \wedge C g$ is stably trivial.

First suppose that $f$ is a stable equivalence. Then $\operatorname{Hom}_{S}(C f, E)$ is a level trivial spectrum which is also injective, by part (4) and the fact that $C f$ is $S$-cofibrant. Therefore $\operatorname{Hom}_{S}(C f \wedge C g, E) \cong \operatorname{Hom}_{S}\left(C g, \operatorname{Hom}_{S}(C f, E)\right)$ is a level trivial spectrum, so $C f \wedge C g$ is stably trivial and thus $f \square g$ is a stable equivalence.

Now suppose that $g$ is a stable equivalence, so that $C g$ is stably trivial. By adjunction $\operatorname{Hom}_{S}(C f \wedge C g, E)=\operatorname{Hom}_{S}\left(C g, \operatorname{Hom}_{S}(C f, E)\right)$. We claim that $D=$ 
$\operatorname{Hom}_{S}(C f, E)$ is an injective $\Omega$-spectrum. Indeed, we have already seen that $D$ is injective. From Remark 2.2.12, we have

$$
\operatorname{Ev}_{n} D \cong \operatorname{Map}_{S p^{\Sigma}}\left(C f \wedge F_{n} S^{0}, E\right) \cong \operatorname{Map}_{S p^{\Sigma}}\left(C f, \operatorname{Hom}_{S}\left(F_{n} S^{0}, E\right)\right) .
$$

Similarly, we have

$$
\left(\operatorname{Ev}_{n+1} D\right)^{S^{1}} \cong \operatorname{Map}_{S p^{\Sigma}}\left(C f, \operatorname{Hom}_{S}\left(F_{n+1} S^{1}, E\right)\right) .
$$

Since $E$ is an $\Omega$-spectrum, $\left(F_{n} S^{0} \wedge \lambda\right)^{*}: \operatorname{Hom}_{S}\left(F_{n} S^{0}, E\right) \rightarrow \operatorname{Hom}_{S}\left(F_{n+1} S^{1}, E\right)$ is a level equivalence. Since $E$ is injective, both the source and target are injective, and so this map is a simplicial homotopy equivalence by Lemma 3.1.6. Hence $\operatorname{Ev}_{n} D \rightarrow\left(\operatorname{Ev}_{n+1} D\right)^{S^{1}}$ is still a level equivalence, so $D=\operatorname{Hom}_{S}(C f, E)$ is an injective $\Omega$-spectrum. Since $C g$ is stably trivial, $\operatorname{Hom}_{S}(C f \wedge C g, E) \cong$ $\operatorname{Hom}_{S}\left(C g, \operatorname{Hom}_{S}(C f, E)\right)$ is a level trivial spectrum, so $C f \wedge C g$ is stably trivial and thus $f \square g$ is a stable equivalence.

Corollary 5.3.8. The projective model structure and the stable model structure on $\mathrm{Sp}^{\Sigma}$ are monoidal.

It also follows that the $S$ model structure on $S p^{\Sigma}$ is monoidal, once it is proven to be a model structure. Neither the injective level structure nor the injective stable structure is monoidal.

Adjunction then gives the following corollary.

Corollary 5.3.9. Let $f$ and $g$ be maps of symmetric spectra.

(1) If $f$ is a stable cofibration and $g$ is a stable fibration, then $\operatorname{Hom}_{\square}(f, g)$ is a stable fibration, which is a level equivalence if either $f$ or $g$ is a stable equivalence.

(2) If $f$ is a stable cofibration and $g$ is a level fibration, then $\operatorname{Hom}_{\square}(f, g)$ is a level fibration, which is a level equivalence if either $f$ or $g$ is a level equivalence.

(3) If $f$ is an $S$-cofibration and $g$ is an $S$-fibration, then $\operatorname{Hom}_{\square}(f, g)$ is an $S$ fibration, which is a level equivalence if either $f$ or $g$ is a stable equivalence.

(4) If $f$ is an $S$-cofibration and $g$ is an injective fibration, then $\operatorname{Hom}_{\square}(f, g)$ is an injective fibration, which is a level equivalence if either $f$ or $g$ is a level equivalence.

(5) If $f$ is a level cofibration and $g$ is an injective fibration, then $\operatorname{Hom}_{\square}(f, g)$ is a level fibration, which is a level equivalence if either $f$ or $g$ is a level equivalence.

Corollary 5.3.10. If $X$ is an $S$-cofibrant symmetric spectrum, the functor $X \wedge-$ preserves level equivalences and it preserves stable equivalences.

Proof. Part (4) of Theorem 5.3.7 implies that $X \wedge-$ preserves level trivial cofibrations. Lemma 4.1.3 then implies that it preserves level equivalences, since every symmetric spectrum is level cofibrant. An arbitrary stable equivalence can be factored as a stable trivial cofibration followed by a level equivalence. Part (5) of Theorem 5.3.7 implies that $X \wedge-$ takes stable trivial cofibrations to stable equivalences.

5.4. The monoid axiom. In [SS97], techniques are developed to form model category structures for categories of monoids, algebras, and modules over a monoidal model category. One more axiom is required which is referred to as the monoid axiom. In this section we verify the monoid axiom for symmetric spectra. The 
results of [SS97] then immediately give a model structure on symmetric ring spectra. After proving the monoid axiom, we discuss the homotopy invariance of the resulting model categories of modules and algebras.

Let $K$ denote the class in $S p^{\Sigma}$ consisting of all maps $f \wedge X$, where $f$ is a stable trivial cofibration and $X$ is some symmetric spectrum. The following theorem implies the monoid axiom for symmetric spectra.

Theorem 5.4.1. Each map in $K$-cof, for $K$ as above, is a stable equivalence.

We then have the following two corollaries, which follow from [SS97, Theorem $3.1]$.

Corollary 5.4.2. Suppose $R$ is a monoid in the category of symmetric spectra. Then there is a model structure on the category of $R$-modules where a map $f: X \rightarrow$ $Y$ is a weak equivalence (fibration) if and only if the underlying map of symmetric spectra is a stable equivalence (stable fibration). Moreover, if $R$ is a commutative monoid, then this is a monoidal model category satisfying the monoid axiom.

Corollary 5.4.3. Suppose $R$ is a commutative monoid in the category of symmetric spectra. Then there is a model structure on the category of $R$-algebras where a homomorphism $f: X \rightarrow Y$ is a weak equivalence (fibration) if and only if the underlying map of symmetric spectra is a stable equivalence (stable fibration). Any cofibration of $R$-algebras whose source is cofibrant as an $R$-module is a cofibration of $R$-modules.

Taking $R=S$ gives a model structure on the category of monoids of symmetric spectra, the symmetric ring spectra.

Proof of Theorem 5.4.1. Each map in $K$ is a level cofibration and a stable equivalence. This follows from parts (3) and (5) of Theorem 5.3.7 applied to an $S$ cofibration and stable equivalence $f$ and the level cofibration $* \rightarrow X$. Let $\Omega$ denote the class of maps $E \rightarrow *$, where $E$ is an injective $\Omega$-spectrum. By part (5) of Corollary 5.3.9 and Proposition 3.1.4, $\operatorname{Hom}_{\square}(K, \Omega)$ consists of level trivial fibrations. Equivalently, $\left(I, \operatorname{Hom}_{\square}(K, \Omega)\right)$ has the lifting property where $I$ is the class of stable cofibrations. Manipulations as in the proof of Proposition 5.3.4 show that $\left(I, \operatorname{Hom}_{\square}(K\right.$-cof, $\left.\Omega)\right)$ also has the lifting property, and so $\operatorname{Hom}_{\square}(K$-cof, $\Omega)$ consists of level trivial fibrations. In particular, this shows that maps in $K$-cof are stable equivalences.

We now show that the model categories of modules and algebras are homotopy invariant.

Lemma 5.4.4. Suppose $R$ is a monoid in $S p^{\Sigma}$, and $M$ is cofibrant in the model category of left $R$-modules. Then $-\wedge_{R} M$ preserves level equivalences and stable equivalences of $R$-modules.

Proof. This is the analogue of Corollary 5.3 .10 for $R$-modules. It follows by proving the analogue of Theorem 5.3.7 for $R$-modules.

Since $S$, the sphere spectrum, is cofibrant in $S p^{\Sigma}$, Theorems 3.3 and 3.4 of [SS97] now apply to give the following theorem.

Theorem 5.4.5. Suppose $f: R \rightarrow R^{\prime}$ is a stable equivalence of monoids of symmetric spectra. Then induction and restriction induce a Quillen equivalence from 
the model category of $R$-modules to the model category of $R^{\prime}$-modules. If, in addition, $R$ and $R^{\prime}$ are commutative, then induction and restriction induce a Quillen equivalence from the model category of $R$-algebras to the model category of $R^{\prime}$ algebras.

5.5. Proper model categories. In this section we recall the definition of a proper model category and show that the stable model category of symmetric spectra is proper.

Definition 5.5.1. (1) A model category is left proper if, for every pushout square

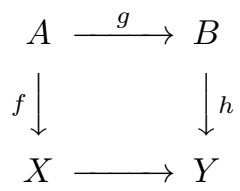

with $g$ a cofibration and $f$ a weak equivalence, $h$ is a weak equivalence.

(2) A model category is right proper if, for every pullback square

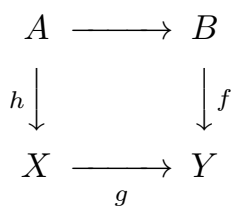

with $g$ a fibration and $f$ a weak equivalence, $h$ is a weak equivalence.

(3) A model category is proper if it is both left proper and right proper.

The category of simplicial sets is a proper model category [BF78] (see [Hir99] for more details). Hence the category of pointed simplicial sets and both level model structures on $S p^{\Sigma}$ are proper.

Theorem 5.5.2. The stable model category of symmetric spectra is proper.

Proof. Since every stable cofibration is a level cofibration, the stable model category of symmetric spectra is left proper by part one of Lemma 5.5.3. Since every stable fibration is a level fibration, the stable model category of symmetric spectra is right proper by part two of Lemma 5.5.3.

Lemma 5.5.3. (1) Let

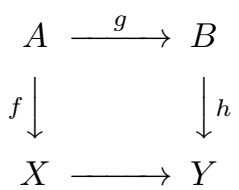

be a pushout square with $g$ a level cofibration and $f$ a stable equivalence. Then $h$ is a stable equivalence.

(2) Let

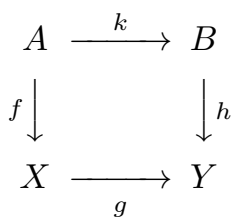

be a pullback square with $g$ a level fibration and $h$ a stable equivalence. Then $f$ is a stable equivalence. 
Proof. Part (1): Let $E$ be an injective $\Omega$-spectrum. Apply the functor $\operatorname{Map}_{S p^{\Sigma}}(-, E)$ to the pushout square. The resulting commutative square

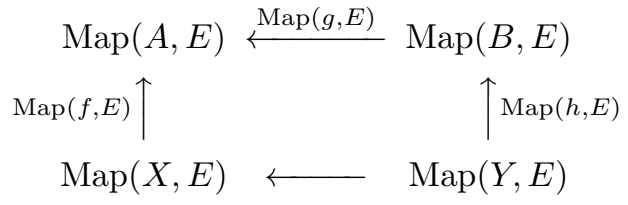

is a pullback square of pointed simplicial sets with $\operatorname{Map}(f, E)$ a weak equivalence, by Proposition 3.1.4. We claim that $\operatorname{Map}(g, E)$ is a Kan fibration. Indeed, let $k: E \rightarrow$ * denote the obvious map, and let $c$ denote a trivial cofibration of pointed simplicial sets. Then $\operatorname{Map}(g, E)=\operatorname{Map}_{\square}(g, k)$. We must show that $\left(c, \operatorname{Map}_{\square}(g, k)\right)$ has the lifting property. By Corollary 3.3.9, this is equivalent to showing that $(c \square g, k)$ has the lifting property. But, by Proposition 1.3.4, $c \square g$ is a level equivalence and a level cofibration. Since $E$ is injective, it follows that $(c \square g, k)$ has the lifting property, and so $\operatorname{Map}(g, E)$ is a Kan fibration. By properness for simplicial sets, $\operatorname{Map}(h, E)$ is a weak equivalence. It follows that $h$ is a stable equivalence.

Part (2): Let $F$ be the fiber over the basepoint of the map $g: X \rightarrow Y$. Since $k$ is a pullback of $g, F$ is isomorphic to the fiber over the basepoint of the map $k: A \rightarrow B$. The maps $X / F \rightarrow Y, A / F \rightarrow B$ and $B \rightarrow Y$ are stable equivalences; so $A / F \rightarrow X / F$ is a stable equivalence. Consider the Barratt-Puppe sequence (considered in the proof of Lemma 3.4.15)

$$
F \rightarrow A \rightarrow A / F \rightarrow F \wedge S^{1} \rightarrow A \wedge S^{1} \rightarrow A / F \wedge S^{1} \rightarrow F \wedge S^{2}
$$

and the analogous sequence for the pair $(F, X)$. Given an injective $\Omega$-spectrum $E$, apply the functor $E^{0}(-)$, and note that $E^{0}\left(Z \wedge S^{1}\right) \cong \pi_{1} \operatorname{Map}(Z, E)$ is naturally a group. The five-lemma then implies that $f \wedge S^{1}: A \wedge S^{1} \rightarrow X \wedge S^{1}$ is a stable equivalence. Part two of Theorem 3.1.14 shows that $f$ is a stable equivalence.

5.6. Semistable spectra. In this section we consider a subcategory of symmetric spectra called the semistable spectra. This subcategory sheds light on the difference between stable equivalences and stable homotopy equivalences of symmetric spectra. As in Section 1.4, the stable homotopy category is equivalent to the homotopy category of semistable spectra obtained by inverting the stable homotopy equivalences. Semistable spectra also play a role in [Shi].

Because stable equivalences are not always stable homotopy equivalences, the stable homotopy groups are not algebraic invariants of stable homotopy types. So the stable homotopy groups of a spectrum may not be "correct". For any symmetric spectrum $X$, though, if there is a map from $X$ to an $\Omega$-spectrum which induces an isomorphism in stable homotopy, then the stable homotopy groups of $X$ are the "correct" stable homotopy groups. In other words, these groups are isomorphic to the stable homotopy groups of the stable fibrant replacement of $X$. Spectra with this property are called semistable.

Let $L$ denote a fibrant replacement functor in $S p^{\Sigma}$, obtained by factoring $X \rightarrow *$ into a stable trivial cofibration followed by a stable fibration, as in Section 4.2.

Definition 5.6.1. A semistable symmetric spectrum is one for which the stable fibrant replacement map, $X \rightarrow L X$, is a stable homotopy equivalence.

Of course $X \rightarrow L X$ is always a stable equivalence, but not all spectra are semistable. For instance, $F_{1} S^{1}$ is not semistable. Certainly any stably fibrant 
spectrum, i.e., an $\Omega$-spectrum, is semistable. In Section 3.1 we defined the functor $R^{\infty}$ and noted that, although it is similar to the standard $\Omega$-spectrum construction for (non-symmetric) spectra, it is not always an $\Omega$-spectrum and $X \rightarrow R^{\infty} X$ is not always a stable homotopy equivalence, even if $X$ is level fibrant. Let $K$ be a level fibrant replacement functor, the prolongation of a fibrant replacement functor for simplicial sets. The following proposition shows that on semistable spectra $R^{\infty} K$ does have these expected properties.

Proposition 5.6.2. The following are equivalent.

(1) The symmetric spectrum $X$ is semistable.

(2) The map $X \rightarrow R K X=\operatorname{Hom}\left(F_{1} S^{1}, K X\right)$ is a stable homotopy equivalence.

(3) $X \rightarrow R^{\infty} K X$ is a stable homotopy equivalence.

(4) $R^{\infty} K X$ is an $\Omega$-spectrum.

Before proving this proposition we need the following lemma.

Lemma 5.6.3. Let $X \in S p^{\Sigma}$. Then $\pi_{k}\left(R^{\infty} K X\right)_{n}$ and $\pi_{k+1}\left(R^{\infty} K X\right)_{n+1}$ are isomorphic groups, and $i_{R^{\infty} K X}:\left(R^{\infty} K X\right)_{n} \rightarrow \operatorname{Hom}\left(F_{1} S^{1}, R^{\infty} K X\right)_{n+1}$ induces a monomorphism $\pi_{k}\left(R^{\infty} K X\right)_{n} \rightarrow \pi_{k+1}\left(R^{\infty} K X\right)_{n+1}$.

Proof. As noted in the proof of Theorem 3.1.11, $\pi_{k}\left(R^{\infty} K X\right)_{n}$ and $\pi_{k+1}\left(R^{\infty} K X\right)_{n+1}$ are isomorphic to the $(k-n)$ th classical stable homotopy group of $X$. However, the map $\pi_{k} i_{R^{\infty} K X}$ need not be an isomorphism. Indeed, $\pi_{k} i_{R^{\infty} K X}$ is the map induced on the colimit by the vertical maps in the diagram

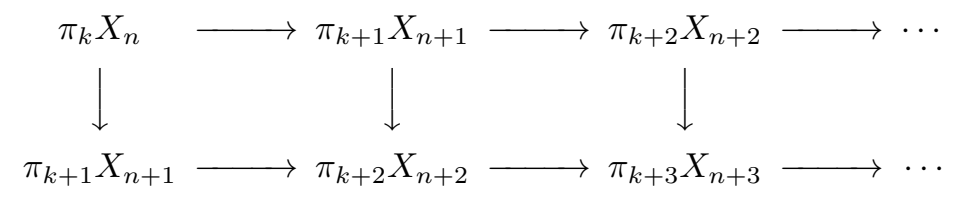

where the vertical maps are not the same as the horizontal maps, but differ from them by isomorphisms. The induced map on the colimit is injective in such a situation, though not necessarily surjective. For an example of this phenomenon, note that we could have $\pi_{k} X_{n} \cong \mathbb{Z}^{n-k}$, with the horizontal maps being the usual inclusions, but the vertical maps begin the inclusions that take $\left(x_{1}, \ldots, x_{n-k}\right)$ to $\left(0, x_{1}, x_{2}, \ldots, x_{n-k}\right)$. Then the element $(1,0, \ldots, 0, \ldots)$ of the colimit is not in the image of the colimit of the vertical maps.

Proof of Proposition 5.6.2. First we show that (1) implies (2) by using the following diagram:

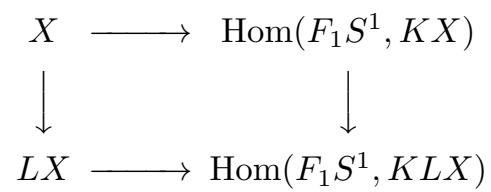

Since $\operatorname{Hom}\left(F_{1} S^{1}, K(-)\right)$ preserves stable homotopy equivalences, both vertical arrows are stable homotopy equivalences. The bottom map is a level equivalence since $L X$ is an $\Omega$-spectrum. Hence the top map is also a stable homotopy equivalence.

Also, (2) easily implies (3). Since $\operatorname{Hom}\left(F_{1} S^{1}, K(-)\right)$ preserves stable homotopy equivalences, $X \rightarrow R^{\infty} K X$ is a colimit of stable homotopy equivalences provided $X \rightarrow \operatorname{Hom}\left(F_{1} S^{1}, K X\right)$ is a stable homotopy equivalence. 
Next we show that (3) and (4) are equivalent. The map $\pi_{*} X \rightarrow \pi_{*} R^{\infty} K X$ factors as $\pi_{*} X \rightarrow \pi_{*}\left(R^{\infty} K X\right)_{0} \rightarrow \pi_{*} R^{\infty} K X$ where the first map here is an isomorphism by definition. Then by Lemma 5.6.3 we see that $\pi_{*}\left(R^{\infty} K X\right)_{0} \rightarrow \pi_{*} R^{\infty} K X$ is an isomorphism if and only if $\pi_{*}\left(R^{\infty} K X\right)_{n} \rightarrow \pi_{*+1}\left(R^{\infty} K X\right)_{n+1}$ is an isomorphism for each $n$.

To see that (3) implies (1), consider the following diagram:

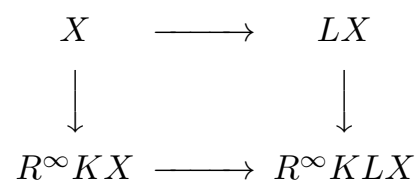

By (3) and (4) the left arrow is a stable homotopy equivalence to an $\Omega$-spectrum, $R^{\infty} K X$. Since $L X$ is an $\Omega$-spectrum the right arrow is a level equivalence. Since $X \rightarrow L X$ is a stable equivalence, the bottom map must also be a stable equivalence. But a stable equivalence between $\Omega$-spectra is a level equivalence, so the bottom map is a level equivalence. Hence the top map is a stable homotopy equivalence.

Two classes of semistable spectra are described in the following proposition. The second class includes the connective and convergent spectra.

Proposition 5.6.4. (1) If the classical stable homotopy groups of $X$ are all finite, then $X$ is semistable.

(2) Suppose that $X$ is a level fibrant symmetric spectrum and there exists some $\alpha>1$ such that $X_{n} \rightarrow \Omega X_{n+1}$ induces an isomorphism $\pi_{k} X_{n} \rightarrow \pi_{k+1} X_{n+1}$ for all $k \leq \alpha n$ for sufficiently large $n$. Then $X$ is semistable.

Proof. By Lemma 5.6.3, $\pi_{k}\left(R^{\infty} K X\right)_{n} \rightarrow \pi_{k+1}\left(R^{\infty} K X\right)_{n+1}$ is a monomorphism between two groups which are isomorphic. In the first case these groups are finite, so this map must be an isomorphism. Hence $R^{\infty} K X$ is an $\Omega$-spectrum, so $X$ is semistable.

For the second part we also show that $R^{\infty} K X$ is an $\Omega$-spectrum. Since for fixed $k$ the maps $\pi_{k+i} X_{n+i} \rightarrow \pi_{k+1+i} X_{n+1+i}$ are isomorphisms for large $i, \pi_{k}\left(R^{\infty} K X\right)_{n} \rightarrow$ $\pi_{k+1}\left(R^{\infty} K X\right)_{n+1}$ is an isomorphism for each $k$ and $n$.

The next proposition shows that stable equivalences between semistable spectra are particularly easy to understand.

Proposition 5.6.5. Let $f: X \rightarrow Y$ be a map between two semistable symmetric spectra. Then $f$ is a stable equivalence if and only if it is a stable homotopy equivalence.

Proof. Every stable homotopy equivalence is a stable equivalence by Theorem 3.1.11. Conversely, if $f$ is a stable equivalence, so is $L f$. Since stable equivalences between stably fibrant objects are level equivalences by Lemma 4.2.6, $L f$ is in particular a stable homotopy equivalence. Since $X$ and $Y$ are semistable, both maps $X \rightarrow L X$ and $Y \rightarrow L Y$ are stable homotopy equivalences. Hence $f$ is a stable homotopy equivalence. 


\section{RefERENCES}

[Ada74] J. F. Adams, Stable homotopy and generalised homology, Chicago Lectures in Mathematics, University of Chicago Press, Chicago, Ill--London, 1974, x+373 pp. MR 53:6534

[Bök85] M. Bökstedt, Topological Hochschild homology, preprint, 1985.

[Bor94] Francis Borceux, Handbook of categorical algebra. 2. Categories and structures, Encyclopedia of Mathematics and its Applications, vol. 51, Cambridge University Press, Cambridge, 1994. MR 96g:18001b

[BF78] A. K. Bousfield and E. M. Friedlander, Homotopy theory of $\Gamma$-spaces, spectra, and bisimplicial sets, Geometric applications of homotopy theory (Proc. Conf., Evanston, Ill., 1977), II (M. G. Barratt and M. E. Mahowald, eds.), Lecture Notes in Math., vol. 658, Springer, Berlin, 1978, pp. 80-130. MR 80e:55021

[Cur71] Edward B. Curtis, Simplicial homotopy theory, Advances in Math. 6 (1971), 107-209. MR 43:5529

[DHK] W. G. Dwyer, P. S. Hirschhorn, and D. M. Kan, Model categories and general abstract homotopy theory, in preparation.

[DS] W. G. Dwyer and Brooke E. Shipley, Hyper symmetric spectra, in preparation.

[DS95] W. G. Dwyer and J. Spalinski, Homotopy theories and model categories, Handbook of algebraic topology (Amsterdam), North-Holland, Amsterdam, 1995, pp. 73-126. MR 96h:55014

[EKMM97] A. D. Elmendorf, I. Kriz, M. A. Mandell, and J. P. May, Rings, modules, and algebras in stable homotopy theory. With an appendix by M. Cole, Mathematical Surveys and Monographs, vol. 47, American Mathematical Society, Providence, RI, 1997, xii+249 pp. MR 97h:55006

[GH97] Thomas Geisser and Lars Hesselholt, Topological cyclic homology of schemes, to appear in K-theory, Seattle, 1996 volume of Proc. Symp. Pure Math.

[Hir99] P. S. Hirschhorn, Localization of model categories, preprint, 1999.

[Hov98a] Mark Hovey, Model categories, Mathematical Surveys and Monographs, vol. 63, American Mathematical Society, Providence, RI, 1998. MR 99h:55031

[Hov98b] Mark Hovey, Stabilization of model categories, preprint, 1998.

[HPS97] Mark Hovey, John H. Palmieri, and Neil P. Strickland, Axiomatic stable homotopy theory, Mem. Amer. Math. Soc. 128 (1997), no. 610. MR 98a:55017

[Lew91] L. Gaunce Lewis, Jr., Is there a convenient category of spectra?, J. Pure Appl. Algebra 73 (1991), 233-246. MR 92f:55008

[Lim59] Elon L. Lima, The Spanier-Whitehead duality in new homotopy categories, Summa Brasil. Math. 4 (1959), 91-148. MR 22:7121

[ML71] S. Mac Lane, Categories for the working mathematician, Graduate Texts in Mathematics, vol. 5, Springer-Verlag, 1971. MR 50:7275

[MMSS98a] M. Mandell, J. P. May, B. Shipley, and S. Schwede, Diagram spaces, diagram spectra and FSPs, preprint, 1998.

[MMSS98b] M. Mandell, J. P. May, B. Shipley, and S. Schwede, Model categories of diagram spectra, preprint, 1998.

[May67] J. P. May, Simplicial objects in algebraic topology, Chicago Lectures in Mathematics, University of Chicago Press, Chicago, Ill., 1967, viii+161pp. MR 36:5942

[Qui67] Daniel G. Quillen, Homotopical algebra, Lecture Notes in Mathematics, vol. 43, Springer-Verlag, 1967. MR 36:6480

[Sch98] Stefan Schwede, S-modules and symmetric spectra, preprint, 1998.

[SS] Stefan Schwede and Brooke Shipley, Classification of stable model categories, in preparation.

[SS97] Stefan Schwede and Brooke Shipley, Algebras and modules in monoidal model categories, to appear in Proc. London Math. Soc.

[Shi] B. Shipley, Symmetric spectra and topological Hochschild homology, to appear in K-theory.

[Voe97] V. Voevodsky, The Milnor conjecture, preprint, 1997.

[Vog70] R. Vogt, Boardman's stable homotopy category, Aarhus Univ. Lect. Notes, vol. 21, Aarhus University, 1970. MR 43:1187 
[Wal85] F. Waldhausen, Algebraic K-theory of spaces, Lecture Notes in Mathematics, vol. 1126, Springer-Verlag, 1985, pp. 318-419. MR 86m:18011

[Whi62] G. W. Whitehead, Generalized homology theories, Trans. Amer. Math. Soc. 102 (1962), 227-283. MR 25:573

Department of Mathematics, Wesleyan University, Middletown, Connecticut 06459

E-mail address: hovey@member.ams.org

Department of Mathematics, Purdue University, West Lafayette, Indiana 47907

E-mail address: bshipley@math.purdue.edu

E-mail address: jhs@math.purdue.edu 